NBER WORKING PAPER SERIES

\title{
IDEA DIFFUSION AND PROPERTY RIGHTS
}

\author{
Boyan Jovanovic \\ Zhu Wang \\ Working Paper 28019 \\ http://www.nber.org/papers/w28019 \\ NATIONAL BUREAU OF ECONOMIC RESEARCH \\ 1050 Massachusetts Avenue \\ Cambridge, MA 02138 \\ October 2020, Revised February 2023
}

We thank Maikol Cerda and Xi Xiong for helping us with this research. The views expressed herein are those of the authors and do not necessarily reflect the views of the Federal Reserve Bank of Richmond or the Federal Reserve System, or the National Bureau of Economic Research.

NBER working papers are circulated for discussion and comment purposes. They have not been peer-reviewed or been subject to the review by the NBER Board of Directors that accompanies official NBER publications.

(C) 2020 by Boyan Jovanovic and Zhu Wang. All rights reserved. Short sections of text, not to exceed two paragraphs, may be quoted without explicit permission provided that full credit, including (C) notice, is given to the source. 
Idea Diffusion and Property Rights

Boyan Jovanovic and Zhu Wang

NBER Working Paper No. 28019

October 2020, Revised February 2023

JEL No. L0

\begin{abstract}
$\underline{\text { ABSTRACT }}$
We study innovation and diffusion of technology at the industry level. We derive an industry's evolution, from birth to its maturity, and we characterize how diffusion affects the incentive to innovate. The model implies that protection of innovators should be only partial due to the congestion externality in the meetings in which idea transfers take place. We fit the model to the early experiences of the automobile and the personal computer industries, both of which show $S$-shaped growth of the number of firms.
\end{abstract}

Boyan Jovanovic

New York University

Department of Economics

19 W. 4th Street, 6th Floor

New York, NY 10012

and NBER

Boyan.Jovanovic@nyu.edu

Zhu Wang

Federal Reserve Bank of Richmond

Research Department

701 East Byrd Street

Richmond, VA 23219

zhuwang001@gmail.com 


\title{
Idea Diffusion and Property Rights
}

\author{
Boyan Jovanovic*and Zhu Wang ${ }^{\dagger}$
}

January 31, 2023

\begin{abstract}
We study innovation and diffusion of technology at the industry level. We derive an industry's evolution, from birth to its maturity, and we characterize how diffusion affects the incentive to innovate. The model implies that protection of innovators should be only partial due to the congestion externality in the meetings in which idea transfers take place. We fit the model to the early experiences of the automobile and the personal computer industries, both of which show $S$-shaped growth of the number of firms.
\end{abstract}

\section{Introduction}

Innovation and diffusion are fundamental drivers of technological progress and longrun growth. An innovation cannot fulfill its potential without being widely adopted, but rapid diffusion and imitation may reduce the incentive to innovate. In this paper, we study the interplay between innovation and diffusion in a competitive industry setting, and discuss welfare and policy implications.

The model features an industry with a fixed demand curve for a homogeneous product and a group of zero measure potential producers. An innovation or "idea" enables an agent to produce the good at zero cost subject to a capacity constraint. At the outset, agents decide whether to pay a sunk cost to innovate. Some will do so immediately; others may consider innovating later, or wait to imitate the innovation.

Imitation occurs in random pairwise meetings between those who have the idea and those who do not. Imitation is costless, but the imitator may have to pay a fee to the idea seller and the fee is determined by the latter's bargaining share.

We study two regimes regarding the payment for ideas. In Regime 1, imitators cannot resell ideas to other imitators. A potential adopter can copy an idea from an

\footnotetext{
*Department of Economics, New York University. Email: bj2@nyu.edu.

${ }^{\dagger}$ Research Department, Federal Reserve Bank of Richmond. Email: zhu.wang@rich.frb.org. The views expressed are solely those of the authors and do not necessarily reflect the views of the Federal Reserve Bank of Richmond or the Federal Reserve System.
} 
imitator but the fee goes to the idea's original innovator and not to the imitator - this scenario is typically seen in patent licensing or franchising. In Regime 2, by contrast, imitators can resell ideas to other imitators and keep the proceeds, a scenario which is often relevant for non-patented know-how.

Our model leads to the following findings. First, under either regime, innovators enter the industry only at the beginning and the number of imitators then follows an $S$-shaped logistic diffusion curve over time. More innovators enter in Regime 1 or when idea sellers' bargaining share is larger, resulting in faster industry growth.

Second, socially optimal compensation for innovators should be only partial. Innovators do generate positive knowledge spillovers, but they also generate a meetingcongestion externality, and from the welfare viewpoint, they should be compensated but not fully. Moreover, the socially optimal bargaining share of idea sellers is larger in Regime 2 where innovators collect the payoff of ideas partly indirectly.

Third, a policy restricting the speed of diffusion reduces welfare. It may encourage entry of innovators and raise initial industry capacity, but it lowers imitation and leads to slower growth of capacity. Non-compete contracts restrict idea diffusion and we show their enforcement in Massachusetts but not in California may explain why venture activity on Route 128 was overtaken by that in Silicon Valley.

We fit the model to the early experiences of the U.S. automobile and personal computer industries, both of which show $S$-shaped growth in the number of producers in the period before the shakeout, a pattern shared by many industries. We thus add to the literature on industry life cycles - e.g., Gort and Klepper (1982), Utterback and Suarez (1993), Jovanovic and MacDonald (1994), Klepper (1996), Filson (2001), and Hayashi, Li, and Wang (2017). Those studies focus on explaining the shakeout of firms, while our study explains the expansion of firm numbers prior to the shakeout. We find that the auto and the PC industries both face highly elastic demands, under which entry of imitators can drive prices down only slowly. Because this encourages innovation and exacerbates the congestion externality, the socially optimal bargaining share of idea sellers should be low for both industries, and lower for the more price elastic PC.

In our model, random meetings between agents who have ideas and those who do not give rise to a logistic diffusion process. This is consistent with prior work that features logistic diffusion curves in the technology diffusion literature (e.g., Griliches 1957, Mansfield 1961, Bass 1969, 2004, Young 2009), ${ }^{1}$ as well as in the epidemics studies (e.g., Atkeson 2020, Garibaldi, Moen, and Pissarides 2020, among many applications of the SIR model to the spread of the COVID-19 disease). And the quadratic matching function underlying the logistic diffusion was recently studied by Lauermann, Nöldeke, and Tröger (2020).

\footnotetext{
${ }^{1}$ Our model focuses on the diffusion process driven by information spillovers from prior to future adopters, which has been a classic approach for studying diffusion in the literature (see Young 2009 for a review). There are also models where diffusion is driven by falling prices of inputs; e.g., David (1968) and Manuelli and Seshadri (2014).
} 
Saxenian (1994), Gilson (1999) and Franco and Mitchell (2008) discuss the role of non-compete contracts in the overtaking of Route 128 by Silicon Valley. Our model generates this overtaking via the discouragement effect that banning non-competes has on innovation and via the offsetting effect on imitation.

We add to several other strands of the literature. First, the work on competitive innovation; Boldrin and Levine (2008) provide evidence that such innovation is pervasive and they argue that in both theory and practice, capacity constraints provide incentives to innovate in a competitive marketplace. They consider a single innovator's entry decision in a market where the number of imitators grows at a constant rate. By contrast, our model endogenizes the entry number of innovators and generates $S$-shaped growth in the number of imitators, and we also consider compensation from imitators to innovators, and our policy implications are different.

Second, our finding that protection of innovators should be only partial agrees with findings in some recent papers on aggregate growth. For example, Hopenhayn and Shi (2020) show that due to matching congestion, the growth-maximizing bargaining share of innovators is sensitive to the parameters in the matching function. Benhabib, Perla, and Tonetti (2021) show that innovators' licensing income becomes highly elastic with regard to the license price when innovators' bargaining power is too strong and that this can lower licensing income, the return to innovation, and growth. These models compare aggregate growth rates at steady states whereas our model studies transitional dynamics of industry evolution and two policy-relevant regimes concerning idea resale.

In our model, innovation generates a payoff that depends partly on the use of the idea in production, and partly on the value the idea yields when it is sold. Idea sales occur in bilateral meetings and our model relates to models in which agents search for a production partner after one has invested, such as Burdett and Coles (2001), Mailath, Samuelson, and Shaked (2000) and Nöldeke and Samuelson (2015). In these models, payoffs in a match depend on partners' investments and this affects investment incentives.

In the model, owners of ideas use them to compete in the product market and thus the flow value of an idea depends on how many others use it. Manea (2021) also assumes ideas are sold in bilateral meetings and uses bargaining to allocate rents, but in his model the flow value of an idea to its user does not depend on how many others have it or use it.

The paper is organized as follows. Section 2 lays out the model and Section 3 characterizes the equilibrium. Section 4 conducts welfare analysis, and Section 5 fits the model to data for the U.S. automobile and personal computer industries. Section 6 analyzes a limiting version of our model that relates to Boldrin and Levine (2008) and Quah (2002), and Section 7 concludes. The proofs of model propositions and robustness checks of empirical studies are in the Appendix. 


\section{Model}

Consider a competitive market in continuous time. There is a measure $N$ of potential producers. At date 0, a measure $k_{0}$ who we call "innovators," each invest an amount $c$ in an innovation that results in the ability to produce one unit of a new good each period at zero cost. They then immediately become producers. After that, the innovation spreads to others. At any date $t \geq 0$ the measure of producers is $k_{t}$, and the remaining $N-k_{t}$ agents are "outsiders." We normalize outsiders' earnings to zero and denote $u_{t}$ as an outsider's option value at date $t$ for entering the industry in the future.

The total output of the homogeneous good is $k_{t}$, and the product price is

$$
p_{t}=A k_{t}^{-\beta}
$$

where $A$ is a market size parameter and $\beta>0$ is the inverse demand elasticity.

Two types of producers. - All producers have the idea and all are equally productive, but some are "innovators" while the others are "imitators." An innovator has paid a direct cost $c$ to invent the idea. An imitator who at date $t$ has copied a producer's idea, has paid a fee equal to

$$
F_{t}=\alpha \omega_{t}
$$

where $\omega_{t}$ is the value of becoming an imitator at $t$. The parameter $\alpha \in[0,1]$ is an idea seller's bargaining share. ${ }^{2}$

\subsection{Diffusion process}

Diffusion occurs through random pairwise meetings between the $k_{t}$ producers and the $N-k_{t}$ outsiders in which outsiders learn and imitate the innovation. The matching function is assumed to be quadratic, and each meeting results in a new producer. An outsider can also enter as an innovator after date 0, but Propositions 1 and 2 will show that no one will want to do so. Thus, for $t>0$ meetings are the only way that agents will in equilibrium become producers, and the number of producers then evolves as

$$
\frac{d k_{t}}{d t}=\gamma k_{t}\left(N-k_{t}\right)
$$

\footnotetext{
${ }^{2}$ Hopenhayn and Shi (2020) show that the bargaining share $\alpha$ could result from an enforcement threat game, in which the firms split the imitator's surplus $\omega_{t}$ from idea transfer. This bargaining protocol is different from Nash bargaining, where the innovator and imitator would split the joint surplus $\omega_{t}-u_{t}$ from the idea transfer. This alternative bargaining is easier to enforce than a Nash bargain because the courts need to know only $\omega_{t}$ and not the imitators' outside options. Section 6 will show that $\alpha$ coincides with the Nash bargaining share in a limiting version of the model where $u_{t}=0$ as $N \rightarrow \infty$.
} 
where $\gamma>0$ is a parameter. The solution to $(3)$ is

$$
k_{t}=\frac{N e^{\gamma N t}}{e^{\gamma N t}+\frac{N}{k_{0}}-1} .
$$

Matching function specification.-The matching function (3) features increasing returns to scale. However, the assumption on returns to scale is inessential for our analysis. $^{3}$ The labor search literature often assumes a Cobb-Douglas matching function:

$$
\frac{d k_{t}}{d t}=\gamma k_{t}^{\theta}\left(N-k_{t}\right)^{1-\theta}
$$

where $0<\theta<1$. However, the Cobb-Douglas formulation does not appear to fit data better, and more importantly, it does not have a closed-form solution for the time path of $k_{t}$. Therefore the logistic formulation has analytical advantages.
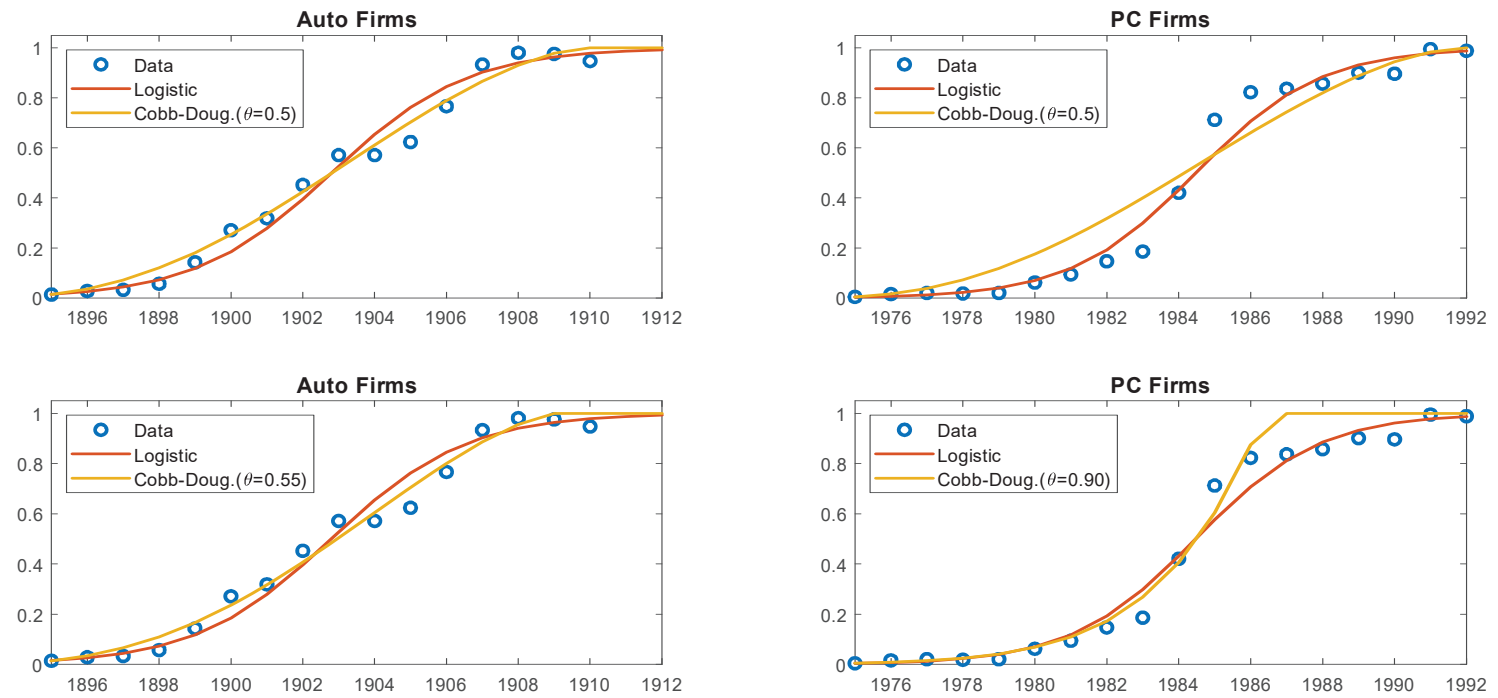

Fig. 1. Diffusion Models: Fitting Time Paths of Firm Numbers

\footnotetext{
${ }^{3}$ To show why, let us generalize Eq. (3) to$$
\frac{d k_{t}}{d t}=\hat{\gamma} k_{t}\left(N-k_{t}\right) \quad \text { where } \quad \hat{\gamma}=\frac{\gamma}{N^{\psi}} .
$$

The solution of $k_{t}$ then becomes

$$
k_{t}=\frac{N e^{\hat{\gamma} N t}}{e^{\hat{\gamma} N t}+\frac{N}{k_{0}}-1}=\frac{N e^{\gamma N^{1-\psi} t}}{e^{\gamma N^{1-\psi} t}+\frac{N}{k_{0}}-1} .
$$

By rescaling the diffusion parameter $\gamma$ with a constant $\frac{1}{N^{\psi}}$, the matching function features increasing returns to scale if $\psi<1$, constant returns if $\psi=1$, and decreasing returns if $\psi>1$. We shall assume that $\psi=0$, but our analysis and findings would hold for any $\psi$ because a time series study takes $N$ and $\frac{\gamma}{N^{\psi}}$ as given; the value of $\psi$ plays no role except in a counterfactual that would involve changing the value of $N$.
} 
Figure 1 shows that the estimated logistic diffusion model (cf. Eq. (4)) matches the time paths of firm numbers well for U.S. automobile and PC industries. Comparing with the symmetric Cobb-Douglas counterpart (i.e., $\theta=0.5$ ), logistic diffusion shows a more pronounced inflection point and fits better for the PC industry, as shown in the top panels. In the bottom panels, we compare logistic diffusion with the best fitting Cobb-Douglas formulation for each industry without restricting $\theta$. The former still fits better for the PC industry. ${ }^{4}$

\subsection{Two regimes}

We shall analyze two regimes that differ in how much revenue innovators get from idea sales.

\section{Regime 1: Imitators cannot resell ideas}

In Regime 1, the original innovators receive all of their ideas' sale revenues; at each date they are divided among the innovators. While an imitator may have learned the innovation from any incumbent producer, he has to pay the idea's original innovator. This type of idea transfer often occurs under franchising or patents that do not allow sublicensing.

Since an imitator cannot resell the innovation, his only revenue comes from selling the good, and his value $\omega_{t}$ satisfies

$$
r \omega_{t}=p_{t}+\frac{d \omega_{t}}{d t}
$$

where $r$ is the interest rate.

An innovator receives revenues from selling both the good and the idea. We will prove that at equilibrium, innovators only enter at date 0 . Accordingly, the number of ideas sold at $t$ is $\gamma k_{t}\left(N-k_{t}\right)$ and the total date- $t$ revenue from these sales, $\gamma k_{t}\left(N-k_{t}\right) \alpha \omega_{t}$, is divided among the $k_{0}$ innovators. Therefore, the date- $t$ value $v_{t}$ of an innovator satisfies

$$
r v_{t}=p_{t}+\frac{\gamma k_{t}\left(N-k_{t}\right)}{k_{0}} \alpha \omega_{t}+\frac{d v_{t}}{d t} .
$$

An outsider's hazard rate for meeting a producer is $\frac{\gamma k_{t}\left(N-k_{t}\right)}{N-k_{t}}=\gamma k_{t}$. Therefore, his lifetime value at date $t, u_{t}$, satisfies

$$
r u_{t}=\gamma k_{t}\left[(1-\alpha) \omega_{t}-u_{t}\right]+\frac{d u_{t}}{d t} .
$$

The free entry condition requires that $v_{t}-u_{t}=c$ for $t=0$.

\footnotetext{
${ }^{4}$ The Cobb-Douglas diffusion curves plotted in Fig. 1 are ones that minimize the sum of the squares of the prediction errors. The data fitting suggests that Cobb-Douglas curves fit slightly better in the auto case $\left(R^{2}=0.985\right.$ when $\theta=0.5$ and $R^{2}=0.987$ when $\left.\theta=0.55\right)$ than the logistic curve $\left(R^{2}=0.975\right)$, but the logistic curve fits better in the PC case $\left(R^{2}=0.981\right)$ than the Cobb-Douglas curves $\left(R^{2}=0.936\right.$ when $\theta=0.5$ and $R^{2}=0.969$ when $\left.\theta=0.90\right)$.
} 


\section{Regime 2: Imitators can resell ideas}

In Regime 2, imitators do get paid for ideas that they resell. An incoming idea buyer pays the agent from whom he copies the idea. This may capture the cases of patents that allow sublicensing and also the spread of non-patented know-how.

Any producer (innovator or imitator) that sells an idea can keep the proceeds. Then all producers now have the same value $v_{t}=\omega_{t}$. Again, we will prove that at equilibrium, innovators only enter at date 0 . The revenue from a single idea sale is $\alpha v_{t}$, and total revenue from idea sales, $\gamma k_{t}\left(N-k_{t}\right) \alpha v_{t}$, is now shared by all the $k_{t}$ producers. Therefore, $v_{t}$ now satisfies

$$
r v_{t}=p_{t}+\gamma\left(N-k_{t}\right) \alpha v_{t}+\frac{d v_{t}}{d t}
$$

The value of an outsider becomes

$$
r u_{t}=\gamma k_{t}\left((1-\alpha) v_{t}-u_{t}\right)+\frac{d u_{t}}{d t} .
$$

Motivation for two regimes. - In a frictionless world, innovators would prefer Regime 2 , as it does not require them to track the idea they sold and enforce the no-reselling constraint on imitators. But in Regime 2 an imitator needs to pay for an idea with a higher fee that incorporates his future revenues from reselling. This can be challenging for new entrants in an emerging industry who often face tight financial constraints. Regime 1 requires a smaller up-front payment by the buyer of the idea each time the idea is transferred. Of course, the use of no-reselling constraint relates to its enforceability; Regime 1 would better reflect patented innovations than non-patented ones.

In our model, imitators' ability to resell ideas or not resell ideas does not affect the meeting process, but it affects the incentive to innovate, and it thus affects market allocation and welfare, as will be shown in the following analysis.

\section{Characterization}

In this section, we characterize equilibrium under each regime.

\subsection{Market equilibrium}

We first solve the equilibrium for each regime. We find that in either Regime 1 or 2 , innovators only enter at date 0 . Accordingly, the time path of firm numbers is given by Eq. (4).

In Regime 1, if a measure-0 outsider were to deviate from the equilibrium and enter as an innovator at date $\tau>0$, its value at date $t \geq \tau$, denoted as $v_{t}^{\tau}$, would satisfy

$$
r v_{t}^{\tau}=p_{t}+\frac{\gamma k_{t}\left(N-k_{t}\right)}{k_{\tau}} \alpha \omega_{t}+\frac{d v_{t}^{\tau}}{d t}
$$


This differs from $v_{t}$ in Eq. (8) because at any date $t \geq \tau$, this new entrant always has a chance $1 / k_{\tau}$ (where $k_{\tau}$ is the number of incumbent firms at his entry date $\tau$ ) to share the industry's total idea sale revenues from new imitators. Because firm numbers continue to rise over time, it does not pay an innovator to enter at a later stage.

Solving the equilibrium for Regime 1, we derive the full dynamic paths of $v_{t}, u_{t}$, $\omega_{t}, v_{t}^{\tau}$ and pin down $k_{0}$ (See Appendix A.1 for details). The results yield Proposition 1. To distinguish Regimes 1 and 2, we will use the superscripts I and II.

Proposition 1 (A) In Regime 1, innovators enter only at date 0. (B) The number of innovators $k_{0}^{\mathbf{I}}$ solves

$$
\underbrace{\frac{1}{N-k_{0}} \int_{0}^{\infty} e^{-r t}\left(\alpha \frac{N}{k_{0}}+(1-\alpha) \frac{N}{k_{t}}-1\right) A k_{t}^{1-\beta} d t}_{v_{0}-u_{0}}=c,
$$

where $k_{t}$ is given by Eq. (4).

Proof. See Appendix A.1.

In Regime 2, if any innovator were to enter the industry after date 0 , he would share the same value $v_{t}$ as an incumbent, be it an innovator or an imitator. The free entry condition requires that $v_{t}-u_{t}=c$ for $t=0$ and one can verify at equilibrium $v_{t}-u_{t}<c$ for any $t>0$ so that even in Regime 2, innovators enter only at date 0 . Solving the equilibrium for Regime 2, we derive the full dynamic paths of $v_{t}, u_{t}$ and pin down $k_{0}$ (See Appendix A.2 for details). The results yield Proposition 2.

Proposition 2 (A) In Regime 2, innovators enter only at date 0. (B) The number of innovators $k_{0}^{\mathbf{I I}}$ solves

$$
\underbrace{\frac{1}{N-k_{0}} \int_{0}^{\infty} e^{-r t}\left(\left(\frac{N}{k_{0}}\right)^{\alpha}\left(\frac{N}{k_{t}}\right)^{1-\alpha}-1\right) A k_{t}^{1-\beta} d t}_{v_{0}-u_{0}}=c
$$

where $k_{t}$ is given by Eq. (4).

Proof. See Appendix A.2.

The isoelastic form for demand in Eq. (1) implies that $k_{0}>0$ in either regime, otherwise $p_{0}$ would be infinite. But additional conditions are needed for $k_{0}$ to be strictly below $N$ :

Proposition 3 In either regime, the entry number of innovators is below $N$ (i.e., $k_{0}^{\mathbf{I}}<N$ and $k_{0}^{\mathbf{I I}}<N$ ) if and only if the following condition holds:

$$
c>\frac{(\alpha \gamma N+r)}{r(r+\gamma N)} A N^{-\beta} .
$$


Proof. See Appendix A.3.

Assuming condition (15) holds, a comparison of Eqs. (13) and (14) yields the findings stated in Propositions 4 and 5:

\section{Proposition 4}

$$
\text { (A) } \quad k_{0}^{\mathbf{I}} \text { and } k_{0}^{\mathbf{I I}}\left\{\begin{array}{c}
\text { increase with } \alpha \text { and } A, \\
\text { decrease with } c \text { and } r .
\end{array}\right.
$$

(B) All parameters being equal across the two regimes,

$$
\frac{k_{0}^{\mathbf{I}}}{k_{0}^{\mathbf{I I}}}=\left\{\begin{array}{ll}
1 & \text { for } \alpha \in\{0,1\} \\
>1 & \text { for } \alpha \in(0,1)
\end{array} .\right.
$$

Proof. See Appendix A.4.

Equation (16) follows because a larger market size $A$ or a higher compensation share $\alpha$ encourages innovation, while a bigger innovation cost $c$ or a higher interest rate $r$ does the opposite. The first line of Eq. (17) holds because the innovators who enter at date 0 either receive no revenue from selling ideas at all (if $\alpha=0$ ) or get all the revenues (if $\alpha=1$ ), and in either scenario whether imitators can or cannot resell the innovation would not matter. For $\alpha \in(0,1)$, however, innovators' revenues get

discounted if they collect the payoff of ideas indirectly, so fewer enter in Regime 2 than in Regime 1. Because the two regimes share the same diffusion process, industry output is higher for all $t$ under Regime 1 due to its larger entry of innovators at date 0 .

Next, we obtain additional insights into how the diffusion rate $\gamma$ affects innovation.

Proposition 5 The effect of the diffusion rate $\gamma$ on innovation $k_{0}^{\mathbf{I}}$ and $k_{0}^{\mathbf{I I}}$ hinges on the values of $\alpha$ and $\beta$. Particularly,

- For inelastic demand $\beta>1$,

$$
k_{0}^{\mathbf{I}} \text { and } k_{0}^{\mathbf{I I}} \text { decrease with } \gamma \text { given that } \beta>1 \geq \alpha .
$$

- For unit elastic demand $\beta=1$,

$$
\begin{aligned}
k_{0}^{\mathbf{I}} \text { and } k_{0}^{\mathbf{I I}} \text { decrease with } \gamma \text { when } \beta & =1>\alpha, \\
k_{0}^{\mathbf{I}} \text { and } k_{0}^{\mathbf{I I}} \text { do not vary with } \gamma \text { when } \beta & =\alpha=1 .
\end{aligned}
$$

- For elastic demand $\beta<1$,

$$
k_{0}^{\mathbf{I}}\left\{\begin{array}{c}
\text { decreases with } \gamma \text { if } 0 \leq \alpha<\frac{1}{\frac{N}{k_{0}^{\mathbf{I}}}(1-\beta)+\beta}<1, \\
\text { increases with } \gamma \text { if } 1 \geq \alpha>\beta+\frac{k_{0}^{\mathbf{I}}}{N}(1-\beta)>0,
\end{array}\right.
$$

and

$$
k_{0}^{\mathbf{I I}}\left\{\begin{array}{l}
\text { decreases with } \gamma \text { if } 0 \leq \alpha<\beta+\frac{k_{0}^{\mathbf{I I}}}{N}(1-\beta)<1 \\
\text { increases with } \gamma \text { if } 1 \geq \alpha>\left(\frac{k_{0}^{\mathbf{I I}}}{N}\right)^{\alpha}(1-\beta)+\beta
\end{array}\right.
$$




\section{Proof. See Appendix A.5.}

The findings of Proposition 5 are intuitive. There are two channels through which diffusion affects innovation. One is the negative price effect, captured by $\beta$ - the faster the diffusion, the lower the revenue from selling the good. Another is the positive idea-selling effect, captured by $\alpha$ - the faster the diffusion, the more ideaselling revenue for the innovators. When demand is inelastic $(\beta>1)$, a faster inflow of imitators would reduce the industry revenue stream, so the price effect dominates and the innovators' value at date 0 would drop even with the highest bargaining share $(\alpha=1)$. This is also true for the unit demand elasticity case when $\alpha<\beta=1$. When demand is elastic $(\beta<1)$, a faster inflow of imitators would increase the industry revenue stream. If $\alpha$ is sufficiently high compared with $\beta$, the idea-selling effect dominates, which raises the incentive to innovate. Otherwise, the price effect dominates which dampens innovation.

\subsection{Market equilibrium: Illustration and applications}

The findings of our model help explain some industry observations and suggest policy impacts.

Intellectual property rights The protection of intellectual property rights raises innovation: Proposition 4 shows that more innovators enter in Regime 1 than in Regime 2 and in each regime, the higher the compensation share $\alpha$, the more innovators enter.

The findings can be visualized using an explicit example. Consider a unit demand elasticity case where $\beta=1$. We normalize $N=1$ and assume $\gamma=r$. Equations (13) and (14) can then be simplified as

$$
\begin{gathered}
\frac{2 k_{0}^{\mathbf{I}}}{(1+\alpha)}=\frac{A}{r c}, \\
\frac{1-k_{0}^{\mathrm{II}}}{\frac{k_{0}^{\mathrm{II}}}{(2-\alpha)\left(N-k_{0}^{\mathrm{II}}\right)}\left(\left(\frac{1}{k_{0}^{\mathrm{II}}}\right)^{2}-\left(\frac{1}{k_{0}^{\mathrm{II}}}\right)^{\alpha}\right)-1}=\frac{A}{r c} .
\end{gathered}
$$

Figure 2 plots the solutions of $k_{0}^{\mathbf{I}}$ and $k_{0}^{\mathbf{I I}}$. The solid lines stand for $k_{0}^{\mathbf{I}}$ and the dashed lines stand for $k_{0}^{\mathrm{II}}$. Cases with different values of $\alpha$ are plotted in different colors. The figure shows that both $k_{0}^{\mathbf{I}}$ and $k_{0}^{\mathbf{I I}}$ increase in $A$ and $\alpha$, but decrease in $c$. It also shows that, $k_{0}^{\mathbf{I}}=k_{0}^{\mathbf{I I}}$ for $\alpha \in\{0,1\}$, and that $k_{0}^{\mathbf{I}}>k_{0}^{\mathbf{I I}}$ for $\alpha \in(0,1)$. Moreover, with the assumed parameter values, condition (15) stated in Proposition 3 can be simplified as $\frac{A}{r c}<\frac{2}{1+\alpha}$, which needs to hold for $k_{0}^{\mathbf{I}}$ and $k_{0}^{\mathbf{I I}}$ to have interior solutions $(<N=1)$ as illustrated by the figure. 


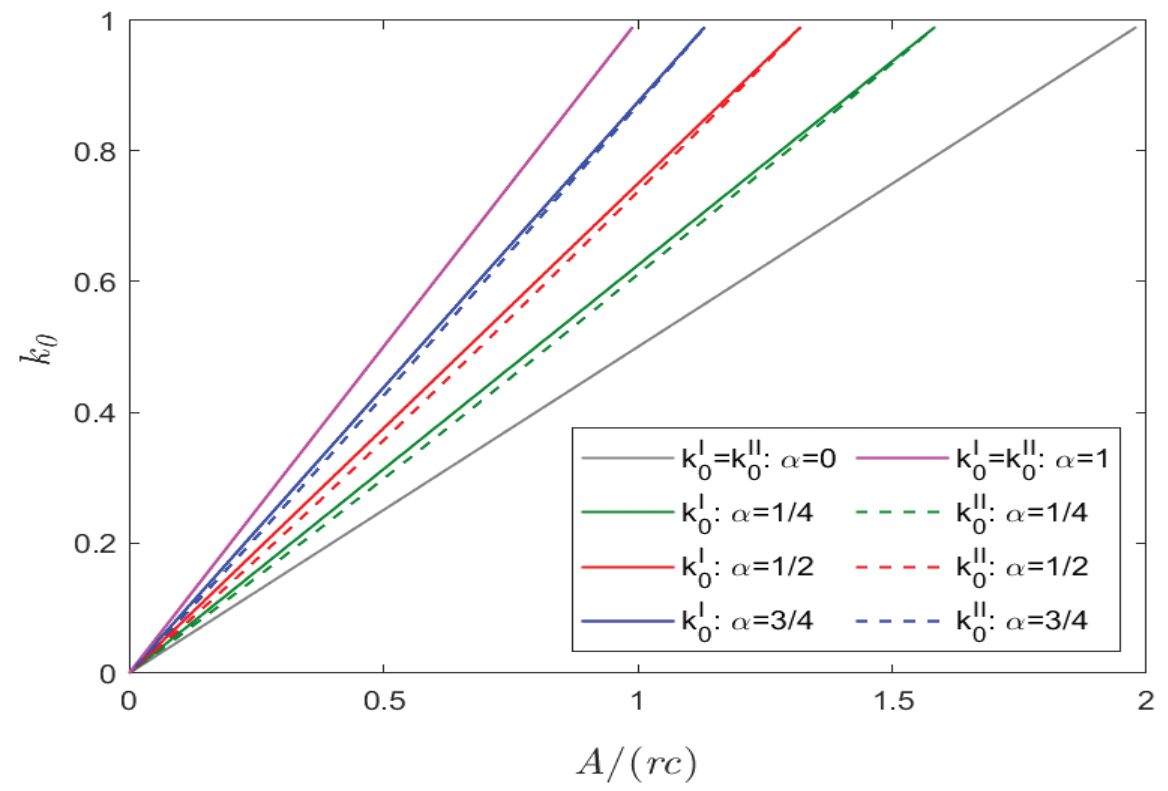

Fig. 2. Entry of Innovators: Model Implications

Restricting entry of imitators Proposition 5 sheds light on the impact of imitation on innovations, which has important bearings on industry policies. For example, one may consider employee spin-offs as imitators, who copy their previous employers' ideas but do not provide sufficient compensation. ${ }^{5}$ In this setting, the diffusion parameter $\gamma$ in our model may reflect the enforcement of non-compete contracts the stricter the enforcement, the lower the $\gamma^{6}{ }^{6}$ Klepper (2010), Samila and Sorenson (2011) and Cabral, Wang, and Xu (2018) find that employee spin-offs lead to industry clusters and that a ban on non-compete contracts is an important contributing factor. According to Saxenian (1994), Gilson (1999) and Franco and Mitchell (2008), because California bans non-compete contracts while Massachusetts enforces them, Silicon Valley overtook Massachusetts' Route 128 in developing high-tech industry.

Indeed, our model shows a mechanism that produces such an overtaking pattern. Suppose that Route 128 and Silicon Valley each specialize in some high-tech sectors, and the two locations face the same environment (i.e., same $A, \alpha, c$ and $N$ ) except that, because California bans non-compete contracts, $\gamma$ is higher than in Massachusetts where non-competes are enforced. Therefore, Route 128 would offer higher

\footnotetext{
${ }^{5}$ While our model does not include labor inputs in production, one may think of employees as people who meet innovators and learn about their innovation. For example, they could work in the same company but do not have to directly produce the new product.

${ }^{6}$ Non-compete contracts require that employees who leave incumbent firms may not conduct business to compete against their previous employers for a period of time. Among others, Shi (2022) analyzes the effects of non-compete contracts which in her model restrict the mobility of managers and reduce welfare. In practice, the enforcement of non-compete contracts varies substantially across the 50 U.S. states (See Bishara, 2011).
} 
incentives to innovators that result in a higher initial entry rate of firms (i.e., a higher $k_{0}$ ) than Silicon Valley. Later on, Silicon Valley's higher imitation rate would lead to its overtaking Route $128 .^{7}$
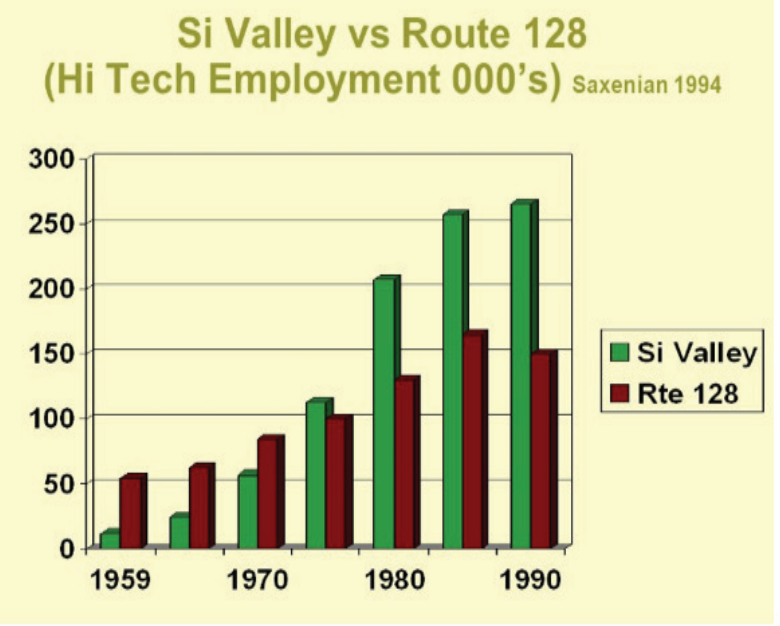

\section{Industry Overtaking: Model Simulation}

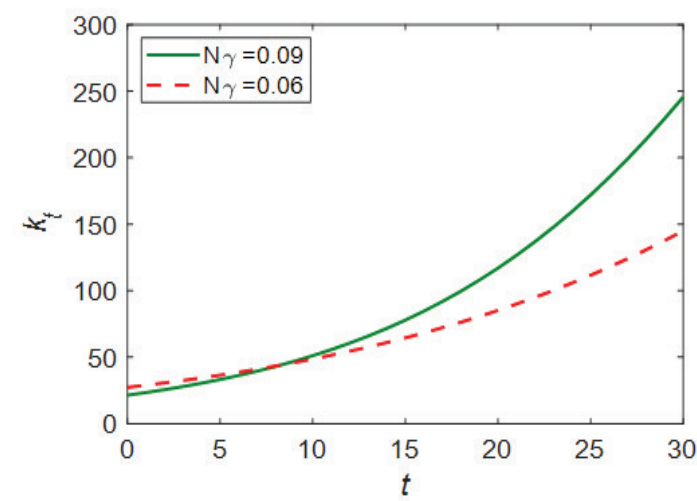

Fig. 3. Industry Overtaking: Data and Model

The left and right panels of Fig. 3 show the data from Saxenian (1994) and our model simulation, respectively. In the simulation, we assume $\beta=1$ and $\alpha=0$ in

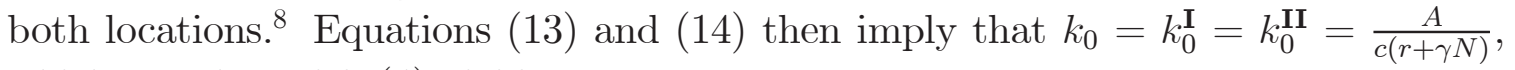
which together with (4) yields

$$
k_{t}=\frac{N e^{\gamma N t}}{e^{\gamma N t}+\frac{N c(r+\gamma N)}{A}-1} .
$$

We assume $A / c=3, N=1000, r=0.05$, and plot $k_{t}$ in two locations: One with a high diffusion rate $(\gamma N=0.09)$, the other with a low one $(\gamma N=0.06)$. As a result, the location with the lower $\gamma$ has more firms early on, but it gets overtaken by the other location after about ten years. In the following welfare analysis, we will show that a higher $\gamma$ also yields higher welfare in this simulation (cf. Proposition 10).

\footnotetext{
${ }^{7}$ Enforcing non-compete contracts may also increase the bargaining share $\alpha$ of innovators. If that happens, the entry of initial innovators will be larger in the enforcing location and the timing of overtaking will be postponed compared with the case where both locations have the same value of $\alpha$.

${ }^{8}$ One could think in one location, non-compete contracts are banned so innovators do not receive any compensation from their employee spin-offs. In another location, non-compete contracts are strictly enforced and the bilateral negotiation to buy out those contracts is too costly for the parties involved, so that spin-off entrants are largely blocked. As a result, both locations have $\alpha=0$ at equilibrium but the diffusion speed $\gamma$ differs.
} 


\section{Welfare analysis}

We now study the welfare implications of the model. Consumers' utility from consuming output $k$ is the integral under the demand curve. For $\beta \in(0,1)$, aggregate utility at output $k$ is

$$
U(k)=\int_{0}^{k} A s^{-\beta} d s=\frac{A}{1-\beta} k^{1-\beta} .
$$

For $\beta \geq 1$ the above integral is infinite; to ensure consumer surplus is finite, we put a maximum, $A \varepsilon^{-\beta}$, on the willingness to pay. Let $D(s)=\min \left(A \varepsilon^{-\beta}, A s^{-\beta}\right)$

and define aggregate utility as $U(k)=\int_{0}^{k} D(s) d s=A\left(\int_{0}^{\varepsilon} \varepsilon^{-\beta} d s+\int_{\varepsilon}^{k} s^{-\beta} d s\right)$ where $\varepsilon \ll k$. Accordingly, for $\beta=1$ we have

$$
U(k)=A(\ln k+1-\ln \varepsilon),
$$

and for $\beta>1$, we have

$$
U(k)=\frac{\beta A}{\beta-1} \varepsilon^{1-\beta}+\frac{A}{1-\beta} k^{1-\beta} .
$$

\subsection{Planner's problem}

The social planner would like to maximize social welfare $W_{0}$ given by

$$
W_{0}=\int_{0}^{\infty} e^{-r t} U\left(k_{t}\right) d t-c k_{0}
$$

where $k_{t}$ satisfies Eq. (4).

Proposition 6 (A) It is socially optimal to innovate only at date 0. (B) The socially optimal number of innovators $k_{0}^{*}$ solves

$$
\underbrace{\int_{0}^{\infty} e^{-(r+\gamma N) t}\left(\frac{k_{t}}{k_{0}}\right)^{2} A k_{t}^{-\beta} d t}_{\text {marginal social return to } k_{0}}=c .
$$

(C) The socially optimal entry number of innovators is an interior solution (i.e., $\left.k_{0}^{*}<N\right)$ if and only if the following condition holds:

$$
c>\frac{A N^{-\beta}}{(r+\gamma N)} .
$$

Proof. See Appendix A.6.

The results of Proposition 6 are intuitive. As of date $\tau \geq 0$, the social return to innovation is

$$
S R_{\tau}=\int_{\tau}^{\infty} e^{-r(t-\tau)} U\left(k_{t}\right) d t
$$


and one can verify that the marginal social return $\partial S R_{\tau} / \partial k_{\tau}$ is strictly decreasing in $k_{\tau}$. So if $k_{0}^{*}$ is chosen so that $\partial S R_{0} / \partial k_{0}=c$ at date 0 , thereafter $\partial S R_{\tau} / \partial k_{\tau}<c$ for any $\tau>0$. Hence, it is socially optimal to innovate only at date 0 . And the condition $\partial S R_{0} / \partial k_{0}=c$ yields Eq. (24). Finally, the social welfare given by Eq. (23) is strictly concave in $k_{0}$, so for $k_{0}^{*}<N$ to hold, one needs

$$
\left.\frac{d W_{0}}{d k_{0}}\right|_{k_{0}=N}<0,
$$

which yields condition (25). This condition is satisfied whenever condition (15) holds. In what follows we shall assume that condition (25) always holds.

Denote the socially optimal welfare by $W_{0}^{*}$. We have the following comparativestatic results.

Proposition 7 All else being equal,

$$
\begin{aligned}
& \text { (A) } k_{0}^{*}\left\{\begin{array}{c}
\text { increases with } A, \\
\text { decreases with } c \text { and } r, \\
\text { decreases with } \gamma \text { if } \beta>1-\frac{k_{0}^{*}}{N-k_{0}^{*}}
\end{array}\right. \\
& \text { (B) } W_{0}^{*}\left\{\begin{array}{r}
\text { increases with } A \text { and } \gamma, \\
\text { decreases with } c \text { and } r .
\end{array}\right.
\end{aligned}
$$

Proof. See Appendix A.7.

Thus $k_{0}^{*}$ and $W_{0}^{*}$ both increase in market size $A$ but decrease in innovation cost $c$ and interest rate $r$. Moreover, $k_{0}^{*}$ decreases with the diffusion rate $\gamma$ if the demand is not too elastic, while $W_{0}^{*}$ always increases with $\gamma$.

\subsection{Three policy instruments}

For a given level of $\gamma$, we now show that a planner can achieve $k_{0}^{*}$ by choosing the bargaining share of idea-sellers, $\alpha$, or by choosing an innovation subsidy (or tax) $s$. And if the planner could raise $\gamma$ by certain policies, we show that doing so would be desirable.

Optimal bargaining share.- Denote the socially optimal bargaining shares for

Regimes 1 and 2 by $\alpha^{\mathbf{I} *}$ and $\alpha^{\mathbf{I I} *}$. A comparison of Eqs. (13), (14) and (24) yields the following result:

\section{Proposition 8}

$$
0<\alpha^{\mathbf{I} *}<\alpha^{\mathbf{I I} *}<1 .
$$




\section{Proof. See Appendix A.8.}

The findings of (28) hold because if $\alpha^{\mathbf{I} *}=\alpha^{\mathbf{I I} *}=0$, no innovator would internalize knowledge spillovers they create for imitators, so fewer innovators enter than the social optimum. On the other hand, if $\alpha^{\mathbf{I} *}=\alpha^{\mathbf{I I} *}=1$, innovators would not fully internalize the congestion externality they impose on one another, so more innovators enter than the social optimum. The congestion arises because an innovator's meeting rate $\frac{d k_{t} / d t}{k_{t}}=\gamma\left(N-k_{t}\right)$ decreases with $k_{t}$ while an imitators' meeting rate $\frac{d k_{t} / d t}{N-k_{t}}=\gamma k_{t}$ increases with $k_{t}{ }^{9}$

Optimal innovation subsidy or tax.-Whenever $\alpha \neq \alpha^{*}$ in each regime, the planner can use a subsidy (or a tax if the subsidy is negative) to achieve the social optimum. Denote the socially optimal subsidy for Regimes 1 and 2 by $s^{\mathbf{I} *}$ and $s^{\mathbf{I} *}$. We obtain the following result:

Proposition 9 Social optimum implies $s^{\mathbf{I} *}<s^{\mathbf{I I} *}$ for $\alpha \in(0,1), s^{\mathbf{I} *}=s^{\mathbf{I I} *}>0$ for $\alpha=0$, and $s^{\mathbf{I} *}=s^{\mathbf{I I} *}<0$ for $\alpha=1$.

Proof. See Appendix A.9.

The intuition for Proposition 9 is as follows. Whenever $\alpha \neq \alpha^{*}$ in each regime, the number of innovators $k_{0}$ differs from the social optimum $k_{0}^{*}$, in which case offering an innovation subsidy (i.e., $s^{*}>0$ whenever $\alpha<\alpha^{*}$ ) or a tax (i.e., $s^{*}<0$ whenever $\left.\alpha>\alpha^{*}\right)$ to adjust the innovation cost $c$ would help restore the social optimum. Recall that when $\alpha \in\{0,1\}$, Regimes 1 and 2 coincide. When $\alpha=0$, too fewer innovators enter than the social optimum, so both regimes would need a positive subsidy to reduce $c$ to achieve $k_{0}^{*}$. When $\alpha=1$, a negative subsidy (i.e., a tax) is needed. Moreover, for $\alpha \in(0,1)$, according to Proposition 4(B), if a given pair of $\alpha$ and $\left(c-s^{\mathbf{I} *}\right)$ lead to the social optimum $k_{0}^{*}$ in Regime 1, the same parameter values would result in a $k_{0}^{\mathbf{I I}}<k_{0}^{*}$ in Regime 2. Therefore, a higher subsidy (or a smaller tax) $s^{\mathbf{I I} *}$ is needed for adjusting $c$ to achieve $k_{0}^{*}$ in Regime 2 given that $k_{0}^{\mathbf{I I}}$ decreases with $c$ as shown by Proposition 4(A).

Optimal diffusion rate.- Suppose that incumbents are not compensated by imitators for spreading ideas, so that $\alpha=0$. From the social welfare point of view, should the planner reduce the diffusion speed $\gamma$ (e.g., by restricting entry of imitators) to enhance incentives for innovation?

\footnotetext{
${ }^{9}$ Assuming a Cobb-Douglas matching function, Hopenhayn and Shi (2020) show that the socially optimal compensation share for innovators should be the innovators' share in the matching function, as in Hosios (1990). However, the same condition would not mechanically apply to our case where we assume a quadratic matching function and solve for the full dynamic path in contrast to a steady state equilibrium. In fact, it is easy to see when $N$ is finite, the parallel to Hosios' condition does not hold. In the quadratic matching function, the shares of $k$ and $N-k$ are equal, which also happens in the Cobb-Douglas case when $\theta=1 / 2$. Yet as we show in this section, the socially optimal $\alpha^{*}$ does not have to be $1 / 2$, and it varies by regime and with other parameters in the model.
} 
Note that if $\alpha=0$, Proposition 5 shows that the entry of innovators decreases with $\gamma$ for any $\beta>0$. Therefore, a policy that reduces the diffusion rate $\gamma$ would boost the entry of innovators. Such policy, however, does not necessarily increase welfare. In fact, we prove the following result for the unit demand elasticity case (i.e., $\beta=1$ ).

Proposition 10 For $\alpha=0$ and $\beta=1$, social welfare always increases with the diffusion rate $\gamma$ for Regimes 1 and 2.

Proof. See Appendix A.10.

Recall that Regimes 1 and 2 coincide when $\alpha=0$ as shown in Proposition 4 . Note that

$$
\frac{d W_{0}}{d \gamma}=\frac{\partial W_{0}}{\partial k_{0}} \frac{\partial k_{0}}{\partial \gamma}+\frac{\partial W_{0}}{\partial \gamma}
$$

From Proposition 8, we know that $\alpha=0$ is below the socially optimal level $\alpha^{*}$, so $\frac{\partial W_{0}}{\partial k_{0}}>0$. Proposition 5 shows that $\frac{\partial k_{0}}{\partial \gamma}<0$ for $\alpha=0$, so $\frac{\partial W_{0}}{\partial k_{0}} \frac{\partial k_{0}}{\partial \gamma}<0$. However, holding $k_{0}$ fixed, $\frac{\partial W_{0}}{\partial \gamma}>0$. Ultimately, Proposition 10 finds that the positive effect of $\frac{\partial W_{0}}{\partial \gamma}$ (i.e., gains from knowledge spillovers) dominates the negative effect of $\frac{\partial W_{0}}{\partial k_{0}} \frac{\partial k_{0}}{\partial \gamma}$ (i.e., disincentives to innovation). This finding suggests that in the numerical example above (cf. Fig. 3), a higher value of $\gamma$ not only helps Silicon Valley overtake Route 128 in industry size, but also yields higher social welfare. In the simulation exercises in Section 4.3 and empirical analysis in Section 5, we find that the result of Proposition 10 actually holds more generally for other values of $\alpha$ and $\beta$.

\subsection{Welfare analysis: Illustration and applications}

We illustrate our welfare analysis with the following examples and applications.

Optimal compensation for idea sellers One can solve for $\alpha^{*} \in(0,1)$ that yields the social optimum. Consider an explicit example used in the above analysis, where $\beta=1, N=1$ and $\gamma=r$. In this example, Eq. (24) simplifies to

$$
\frac{\left(1-k_{0}^{*}\right)^{2}}{\frac{1}{k_{0}^{*}}-1-\ln \frac{1}{k_{0}^{*}}}=\frac{A}{c r} \text {. }
$$

In Regime 1 where imitators cannot resell the innovation, Eqs. (18) and (30) imply that

$$
\alpha^{\mathbf{I} *}=\frac{2\left(1-k_{0}^{*}+k_{0}^{*} \ln k_{0}^{*}\right)}{\left(1-k_{0}^{*}\right)^{2}}-1 .
$$

Alternatively, in Regime 2 where imitators can resell ideas, Eqs. (19) and (30) imply that $\alpha^{\mathbf{I I} *}$ solves

$$
\frac{1}{\frac{k_{0}^{*}}{(2-\alpha)\left(1-k_{0}^{*}\right)}\left(\left(\frac{1}{k_{0}^{*}}\right)^{2}-\left(\frac{1}{k_{0}^{*}}\right)^{\alpha}\right)-1}=\frac{1-k_{0}^{*}}{\frac{1}{k_{0}^{*}}-1-\ln \frac{1}{k_{0}^{*}}} .
$$



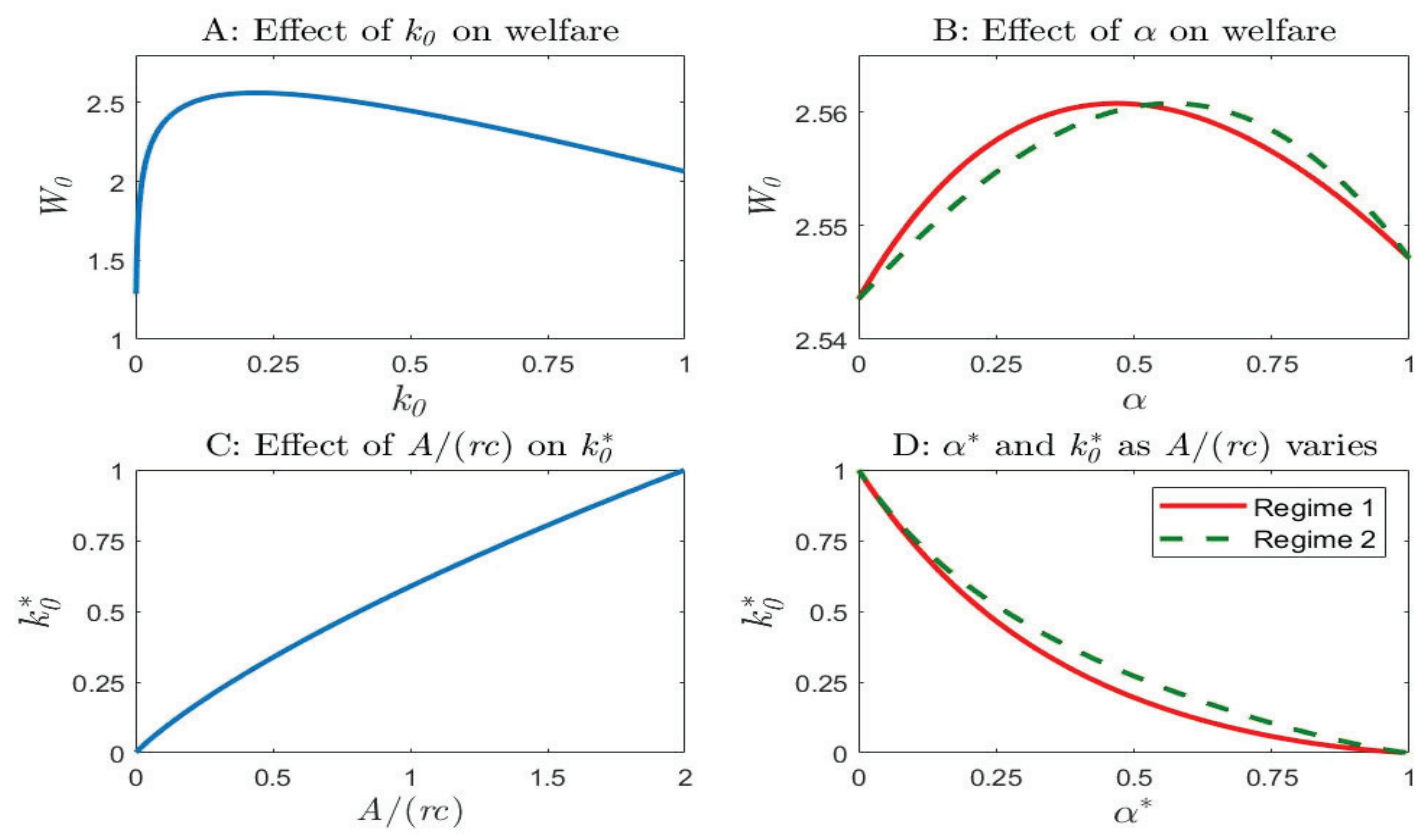

Fig. 4. Properties of the Social Optimum

Figure 4 illustrates this example. Assuming $A /(r c)=0.3$, Fig. 4 A plots the relation between $W_{0}$ and $k_{0}$, given by Eq. (23). ${ }^{10}$ The result shows that welfare maximizes at $k_{0}^{*}=0.22$. Note that Fig. $4 \mathrm{~A}$ has the planner controlling $k_{0}$ directly, so $\alpha$ plays no role. Figure $4 \mathrm{~B}$ shows that this welfare maximum can be implemented via market equilibrium by either setting $\alpha^{\mathbf{I} *}=0.47$ under Regime 1 or setting $\alpha^{\mathbf{I I} *}=0.57$ under Regime 2. Figures $4 \mathrm{C}$ and $4 \mathrm{D}$ extend the results to the full domain of $A /(r c)$ where $k_{0}^{*}$ has an interior solution. Figure $4 \mathrm{C}$ plots the relation between $k_{0}^{*}$ and $A /(r c)$ given by Eq. (30), and Fig. $4 \mathrm{D}$ traces out the relation between $k_{0}^{*}$ and $\alpha^{*}$ that satisfies Eqs. (31) or (32). The negative relation between two endogenous variables, $k_{0}^{*}$ and $\alpha^{*}$, is induced by changes in $A /(r c)$ - as $A /(r c)$ rises, so does $k_{0}^{*}$ but $\alpha^{*}$ falls. ${ }^{11}$ For a given value of $k_{0}^{*}$ (or the corresponding $A /(r c)$ ), the value of $\alpha^{\mathbf{I} *}$ is always smaller than $\alpha^{\mathbf{I I} *}$.

Optimal innovation subsidy or tax Alternatively, if the planner does not control $\alpha$, he could use an innovation subsidy if $\alpha$ is below $\alpha^{*}$ or an innovation tax if $\alpha>\alpha^{*}$.

Consider again the case where $\beta=1, N=1$ and $\gamma=r$. In Fig. 5A, we plot Eq. (30) using a black solid line and overlay it on Fig. 2 introduced in Section 3.2. Figure

\footnotetext{
${ }^{10}$ Equations (21) and (23) show that $c$ and $\varepsilon$ are just scaling parameters and they do not affect the maximization of $W_{0}$, so without loss of generality we set $c=1$ and $\varepsilon=0.0001$ for plotting Figs. $4 \mathrm{~A}$ and $4 \mathrm{~B}$.

${ }^{11}$ Intuitively, a lower $c$ or a higher $A$ leads to a higher $k_{0}^{*}$ at the social optimum, and because such conditions also encourage entry of innovators at market equilibrium and exacerbate the congestion externality, they would require a lower $\alpha^{*}$ to achieve $k_{0}^{*}$ (see Section 5.3.1 for more discussion of the comparative statics for $\alpha^{*}$ ).
} 
$5 \mathrm{~A}$ shows that for a given level of $\frac{A}{r c}, k_{0}^{*}$ always exceeds the market equilibrium level (i.e., $k_{0}^{\mathbf{I}}$ or $k_{0}^{\mathbf{I I}}$ ) when $\alpha=0$, but falls short when $\alpha=1$. Moreover, for any value of $\alpha \in(0,1)$, the socially optimal entry $k_{0}^{*}$ can be achieved by adding an appropriate subsidy or tax to $c$. In the figure, the difference between a market equilibrium path (associated with a particular $\alpha$ and a regime) and the socially optimal path indicates the amount of adjustment to $\frac{A}{r c}$ (i.e., $\frac{A}{r\left(c-s^{I *}\right)}$ or $\frac{A}{r\left(c-s^{I+*}\right)}$ ) needed to achieve each socially optimal level of $k_{0}^{*}$. Figure 5B plots the subsidy (scaled by the innovation $\operatorname{cost} c$ ) needed to achieve the social optimum. The figure shows that the scaled subsidies, $s^{\mathbf{I} *} / c$ and $s^{\mathbf{I} \mathbf{*}} / c$, both decrease in $\frac{A}{r c}$ and $\alpha$, and can turn negative (i.e., become taxes) if $\frac{A}{r c}$ or $\alpha$ becomes sufficiently large. Moreover, $s^{\mathbf{I} *}=s^{\mathbf{I I} *}>0$ (i.e., a subsidy) for $\alpha=0, s^{\mathbf{I} *}=s^{\mathbf{I} *}<0$ (i.e., a tax) for $\alpha=1$, and $s^{\mathbf{I} *}<s^{\mathbf{I} *}$ for $0<\alpha<1$.
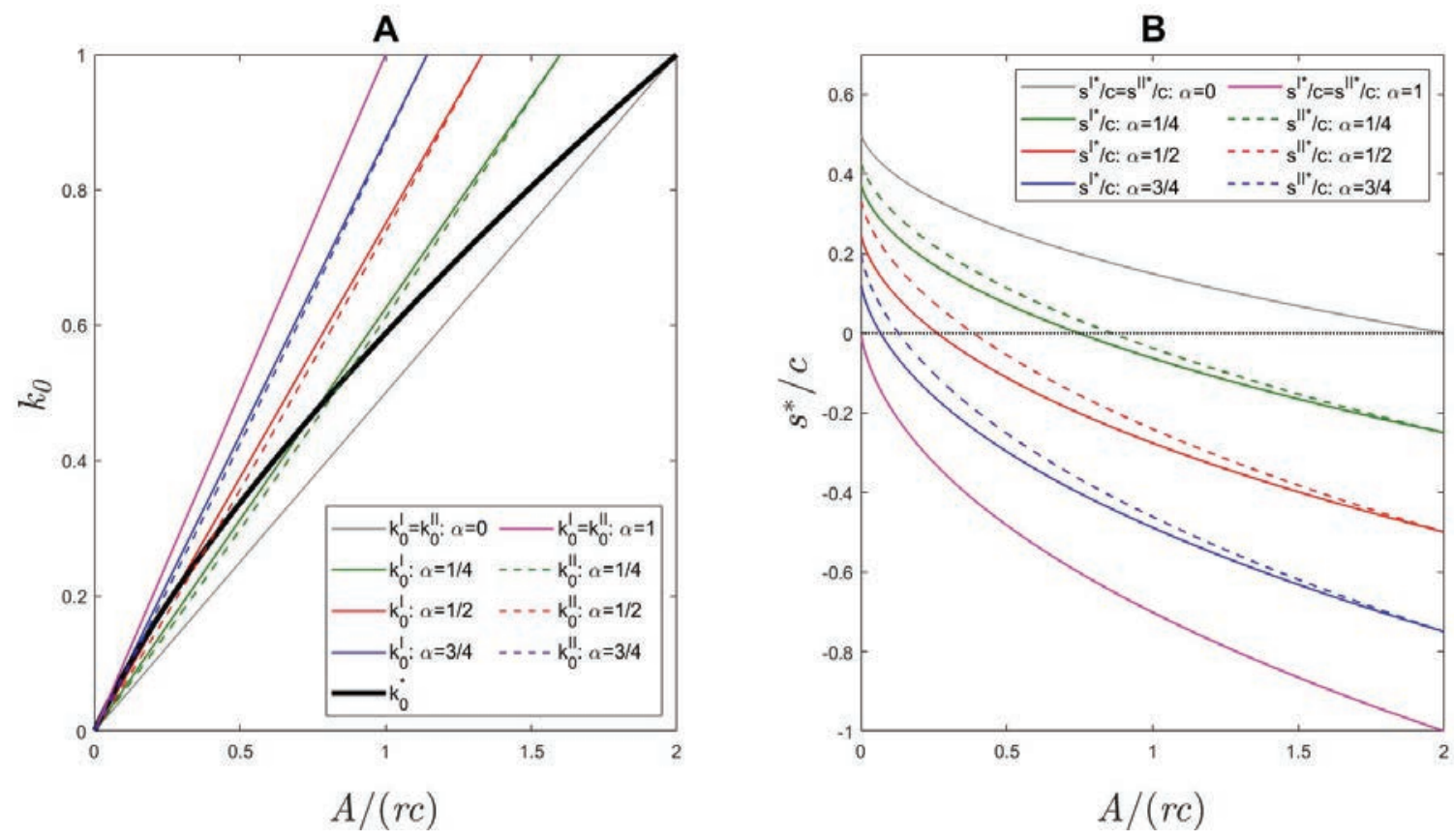

Fig. 5. Socially Optimal Subsidy or Tax

Optimal diffusion rate Our model also sheds light on diffusion policies. Proposition 10 shows that from the social welfare point of view, the planner may not want to slow down the diffusion speed $\gamma$ (e.g., by restricting entry of imitators) even when it could enhance incentives for innovation.

Figure 6 extends the discussion to other values of $\alpha$ for the unit elastic demand case (i.e., $\beta=1$ ). ${ }^{12}$ The figure shows that in both Regimes 1 and 2, for any $\alpha$ in the unit interval, a lower diffusion rate $\gamma$ raises the entry of innovators $k_{0}$ but always lowers social welfare $W_{0}$. In fact, one can prove the result formally for Regime 1 .

\footnotetext{
${ }^{12}$ For illustration, we assume $N=1, A /(r c)=0.3, r=0.05$ in the simulation, and we compare a high $\gamma(\gamma=0.5)$ case versus a low $\gamma(\gamma=0.25)$ case.
} 
Proposition 11 For $\beta=1$ and for any $\alpha \in[0,1]$, social welfare always increases with the diffusion rate $\gamma$ in Regime 1.

Proof. See Appendix A.11.
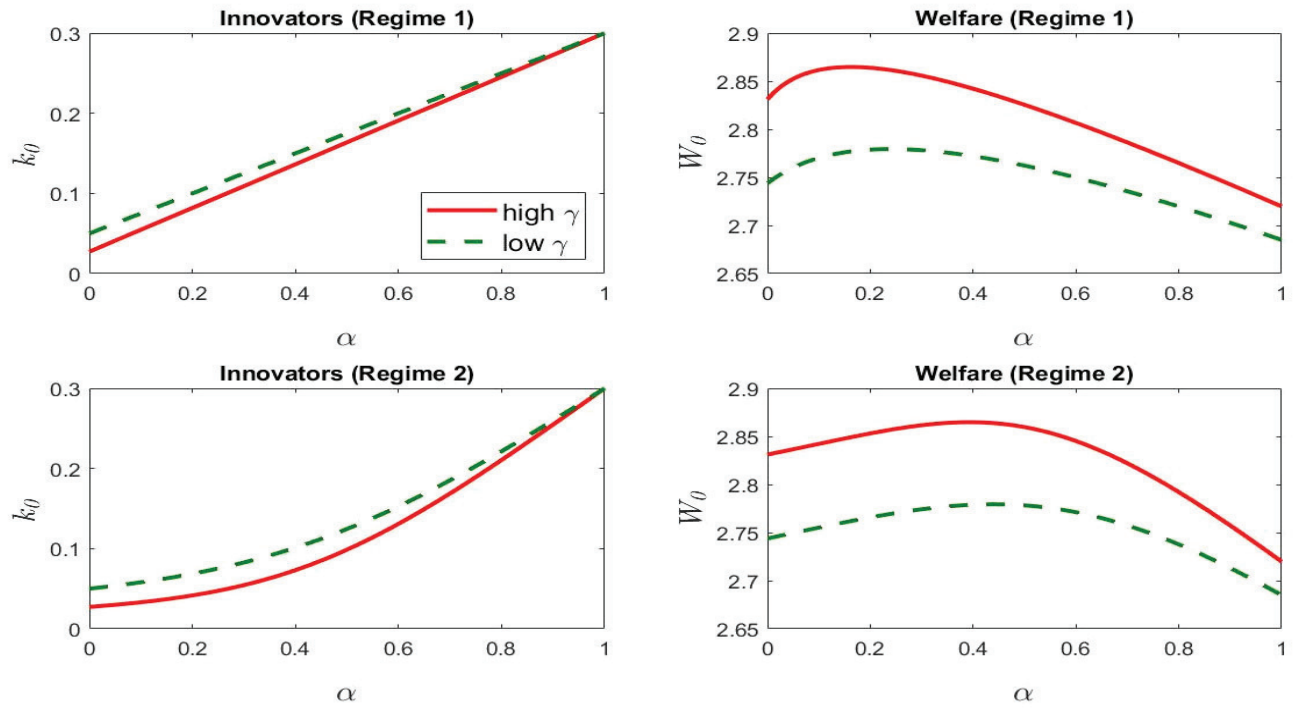

Fig. 6. Effects of the Diffusion Rate $\gamma$

This exercise suggests that the planner may not want to slow down diffusion by lowering $\gamma$ even when $k_{0}^{\mathbf{I}}$ and $k_{0}^{\mathbf{I I}}$ are below $k^{*}$. Rather, the planner should address the compensation to innovators (i.e., $\alpha$ ) directly. This finding highlights the importance of technology diffusion to welfare and lends support to public policies that accommodate diffusion. In Section 5, we carry the analysis to empirical studies on the U.S. auto and $\mathrm{PC}$ industries where demands are price elastic (i.e., $\beta<1$ ) and show this finding continues to hold. ${ }^{13}$

\section{$5 \quad$ Empirical applications}

In this section, we apply our model to data. We consider two historically important industries: automobile and personal computer, where idea diffusion played an important role in the industries' development. ${ }^{14}$ Using model calibration and counterfactual exercises, we evaluate and quantify our theoretical predictions.

\footnotetext{
${ }^{13}$ Note that the finding does not rule out the possibility that policymakers can exploit the welfare gain of temporarily restricting $\gamma$. For example, policymakers could promise to restrict $\gamma$ initially to achieve the socially optimal entry of innovators $k^{*}$, and then free up the limitation. However, such a policy is time-inconsistent and would be futile if market participants anticipate that ex post policymakers cannot commit to that promise (Kydland and Prescott, 1977). Presumably policy must apply more broadly, not just to one instance, but to future products and future instances of $k$.

${ }^{14}$ E.g., Klepper (2010) documents how the spawning of employee spin-offs and entry by firms in related industries drove the development of the automobile and the semiconductor industries.
} 


\subsection{Parameter estimation}

We first estimate the model parameters using auto and PC industry data. The data comes from the following sources:

Auto.- Smith (1970) lists every make of passenger cars produced commercially in the United States from 1895-1969. Smith's list of car makes is used to derive the number of auto firms each year. Thomas (1977) provides annual data of average car price and output from 1900-1929.

PC.-Firm numbers are from Stavins (1995) and the Thomas Register of American Manufacturers, which include desktop and portable computers. Price and quantity information is from the Information Technology Industry Data Book.

In addition, Williamson (2020) provides annual data of U.S. population, real GDP, and the GDP deflator.

\subsubsection{Auto Industry}

The U.S. automobile industry started in 1890s and grew from a small infant industry to a major sector of the economy in a few decades. Starting with 3 firms in 1895, the number of auto producers exceeded 200 around 1910. A shakeout then followed when a major process innovation, the assembly line, was introduced in the early 1910s. As a result, the number of firms declined sharply while the industry output expanded tremendously. Eventually, only 24 firms survived into 1930s. Figure 7 plots the number of firms and output per firm in the U.S. auto industry from 1895-1929.

Our model describes the auto industry development well for the pre-shakeout period (1895-1910). As shown in Fig. 7, during that period, the time path of firm numbers followed an $S$-shaped curve and the average output per firm stayed flat which reflects firms' production capacity constraint. To calibrate the model, we focus on the pre-shakeout era. We assume the shakeout to be an unexpected shock in the benchmark analysis, and we then extend the model to incorporate the shakeout as an anticipated shock in Section 5.4.2.
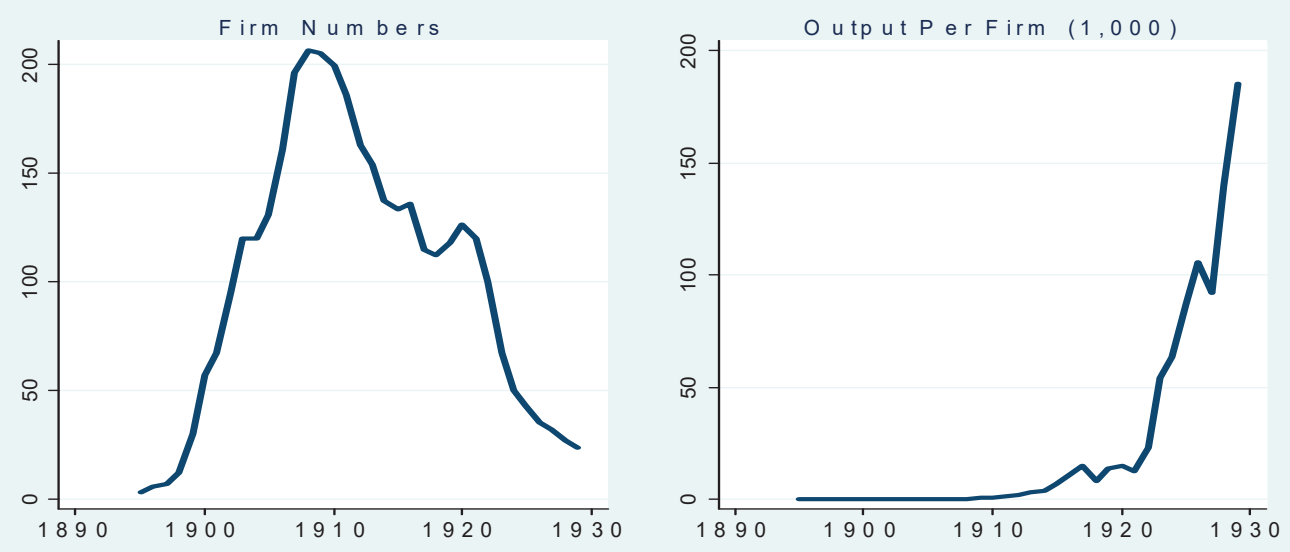

Fig. 7. Auto Firm Numbers and Output Per Firm 
Diffusion estimation We first use the data of firm numbers in the pre-shakeout period, 1895-1910, to estimate the diffusion parameters. In doing so, we rewrite Eq. (4) to estimate the diffusion process of $k_{t}$ as follows:

$$
\ln \frac{k_{t}}{N-k_{t}}=z+\lambda t
$$

where $z=\ln \frac{k_{0}}{N-k_{0}}$, and $\lambda=\gamma N .^{15}$

We assume that the shakeout started after almost all the potential firms had entered the industry. Accordingly, we set $N=210$ and run the regression model. ${ }^{16}$ The result shows that

$$
\ln \frac{k_{t}}{N-k_{t}}=\underset{(0.26)^{* * *}}{-4.13}+\underset{(0.03)^{* * *}}{0.53} t
$$

and the standard errors are reported in the parentheses. The estimates of $z$ and $\lambda$ are both statistically significant at $1 \%$ level (noted by three stars), and adjusted $R^{2}=0.96$. The fit of estimation is shown in Fig. 8. Based on the estimates of diffusion parameters, we calibrate $\gamma N=0.53$ and $k_{0}=3.31$ (i.e., $\ln \frac{k_{0}}{N-k_{0}}=-4.13$ ).

For robustness checks, we also estimated the diffusion process using the matching function (3) which allows differencing the data. The regression results, reported in Appendix B.1, are consistent with the estimates above.

Demand estimation We then estimate the auto demand function using annual data of real auto prices $p_{t}$ (in 2012 price) and industry output $Q_{t}$ from 1900-1929. Equation (1) suggests a simple log-log demand function:

$$
\ln \left(Q_{t}\right)=a-\phi \ln \left(p_{t}\right) .
$$

To address potential endogeneity of the price variable, we use the output per firm (lagged by a year) as an instrumental variable to estimate the demand elasticity parameter $\phi$ in a two-stage least-squares regression. Output per firm, while assumed fixed in our theory, did grow over the long term in data due to technological progress. As a result, it can serve as a valid supply shifter to trace out the demand curve.

The first-stage regression result (adj. $R^{2}=0.87$ ) is given by

$$
\ln \left(p_{t}\right)=\underset{(0.14)^{* * *}}{11.37}-\underset{(0.02)^{* * *}}{0.24} \times \ln (\text { output per firm })_{t-1},
$$

and the second-stage regression result $\left(R^{2}=0.82\right)$ is

$$
\ln \left(Q_{t}\right)=\underset{(2.75)^{* * *}}{47.05}-\underset{(0.29)^{* * *}}{3.61} \times \ln \left(p_{t}\right) .
$$

All the estimates are statistically significant at $1 \%$ level (noted by three stars). The fit of estimation is shown in Fig. 8.

\footnotetext{
${ }^{15}$ Note that Eq. (4) implies $\frac{k_{t}}{N-k_{t}}=\frac{e^{\gamma N t}}{\frac{N}{k_{0}}-1}$, which leads to Eq. (33).

${ }^{16}$ We try an alternative assumption for $N$ in Section 5.4.1 as a robustness check.
} 

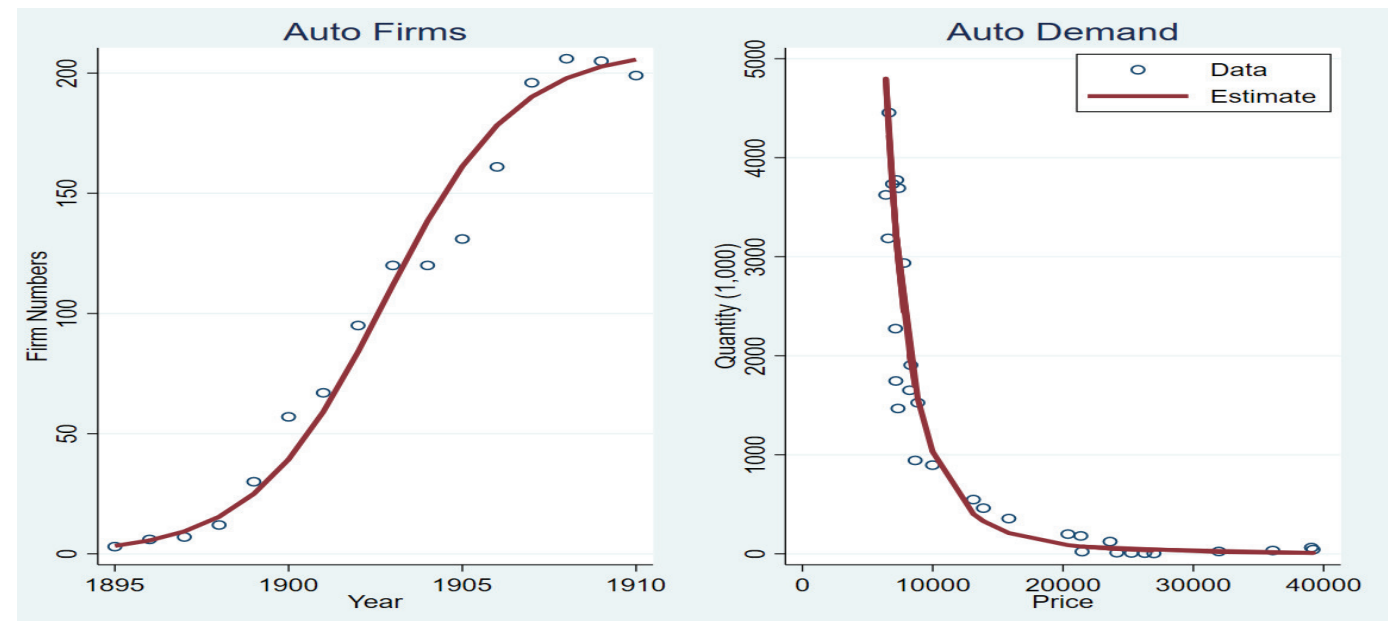

Fig. 8. Auto Diffusion and Demand Estimates

The IV estimation gives $\phi=3.61$ and $a=47.05$. Because our model specifies an inverse demand function (1) that implies

$$
\ln Q_{t}=\frac{1}{\beta} \ln \tilde{A}-\frac{1}{\beta} \ln p_{t},
$$

this yields that $\beta=0.28$ (i.e., $\frac{1}{\beta}=\phi=3.61$ ) and $\tilde{A}=45,737$ (i.e., $\frac{1}{\beta} \ln \tilde{A}=47.05$ ). ${ }^{17}$

For robustness checks, we also re-ran the IV regressions by controlling changes of population and per capita income over time, and the results are very similar (see Appendix B.2). Cabral, Wang and Xu (2018) estimated the auto demand function for the same sample period. They used a different instrumental variable, the share of spin-off firms in the auto industry. The idea is that the founders of spin-off firms are more experienced than de novo entrants, so spin-off firms tend to perform better (Klepper, 2010). They show that their instrument variable performs well and the estimated demand elasticity $\phi=3.39$, which is very close to ours.

\subsubsection{PC industry}

The personal computer industry was developed 80 years later than the automobile industry, but the industry evolution was not much different. Starting with two firms in 1975, the number of PC producers exceeded 430 in 1992. A shakeout then started when the number of firms fell sharply while the industry output continued to expand. Figure 9 plots the number of firms and output per firm in U.S. PC industry from 1975-1999. Like in the auto industry case, our model describes the pre-shakeout period (1975-1992) of the PC industry well. As shown in Fig. 9, during that period, the time path of firm numbers followed an $S$-shaped curve and the average output per firm stayed flat.

\footnotetext{
${ }^{17}$ In the model, we normalize a firm's output to 1 , so $Q_{t}=k_{t}$ and the inverse demand function is $p_{t}=A k_{t}^{-\beta}$. In the empirical analysis, we denote a firm's output by $q$, so $Q_{t}=q k_{t}$ and the corresponding inverse demand function becomes $p_{t}=\tilde{A} Q_{t}^{-\beta}$.
} 

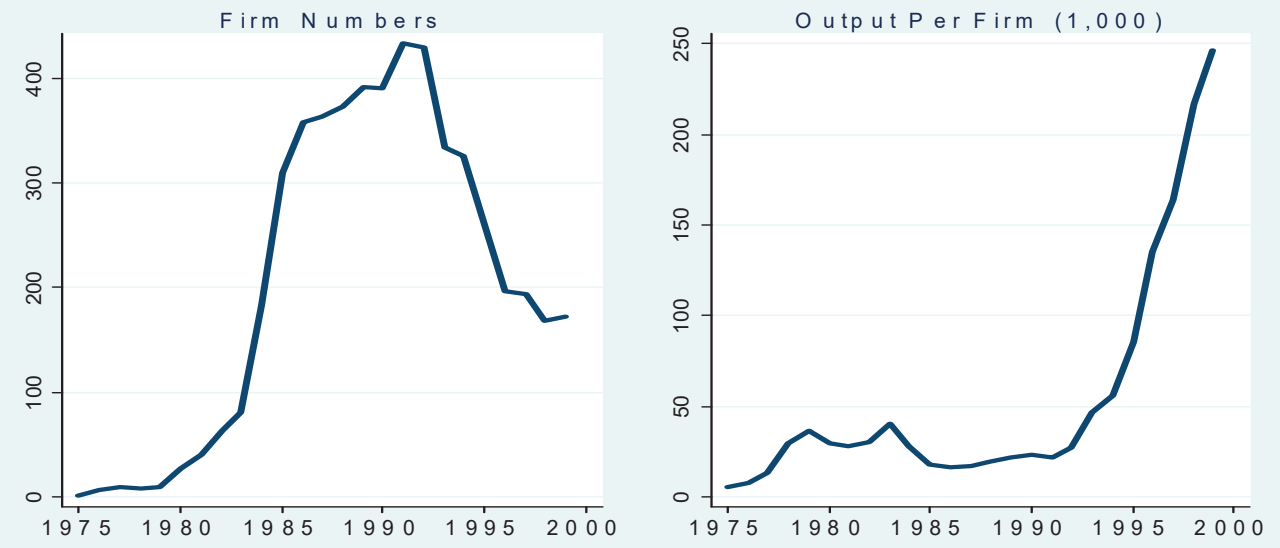

Fig. 9. PC Firm Numbers and Output Per Firm

Diffusion estimation We first use the data of firm numbers in the pre-shakeout period, 1975-1992, to estimate the diffusion parameters. We assume the shakeout started after almost all the potential PC firms had entered the industry. Accordingly, we set $N=435$ and run the following regression model (36). ${ }^{18}$ The result shows that

$$
\ln \frac{k_{t}}{N-k_{t}}=\underset{(0.29)^{* * *}}{-5.49}+\underset{(0.03)^{* * *}}{0.58} t,
$$

with the standard errors reported in the parentheses. All the coefficient estimates are statistically significant at $1 \%$ level, and adjusted $R^{2}=0.96$. The fit of estimation is shown in Fig. 10. Based on the estimates of diffusion parameters, we calibrate $\gamma N=0.58$, and $k_{0}=1.78$ (i.e., $\ln \frac{k_{0}}{N-k_{0}}=-5.49$ ).

For robustness checks, we also estimated the diffusion process using the matching function (3) which allows differencing the data. The regression results, reported in Appendix B.3, are consistent with the estimates above.

Demand estimation We then estimate the PC demand function using annual data of real PC prices $p_{t}$ (in 2012 price) and industry output $Q_{t}$ from 1975-1992. As before, in order to address potential endogeneity of the price variable, we use average output per firm (lagged by a year) as an instrumental variable to estimate the demand elasticity $\phi$.

The first-stage regression result (adj. $R^{2}=0.23$ ) is given by

$$
\ln \left(p_{t}\right)=\underset{(0.50)^{* * *}}{9.62}-\underset{(0.05)^{* *}}{0.12} \times \ln (\text { output per firm })_{t-1},
$$

and the second-stage regression result $\left(R^{2}=0.94\right)$ is

$$
\ln \left(Q_{t}\right)=\underset{(12.52)^{* * *}}{137.15}-\underset{(1.49)^{* * *}}{14.58} \times \ln \left(p_{t}\right) .
$$

\footnotetext{
${ }^{18}$ We try an alternative assumption for $N$ in Section 5.4.1 as a robustness check.
} 
Standard errors are reported in the parentheses, with two and three stars indicating statistical significance at $5 \%$ and $1 \%$ levels, respectively. The fit of estimation is shown in Fig. 10.

The IV estimation gives $\phi=14.58$ and $a=137.15$. This yields $\beta=0.07$ (i.e., $\frac{1}{\beta}=\phi=14.58$ ) and $\tilde{A}=12,170$ (i.e., $\frac{1}{\beta} \ln \tilde{A}=a=137.15$ ). For robustness checks, we also re-ran the IV regressions by controlling changes of population and per capita income over time, and the results are very similar (see Appendix B.4).
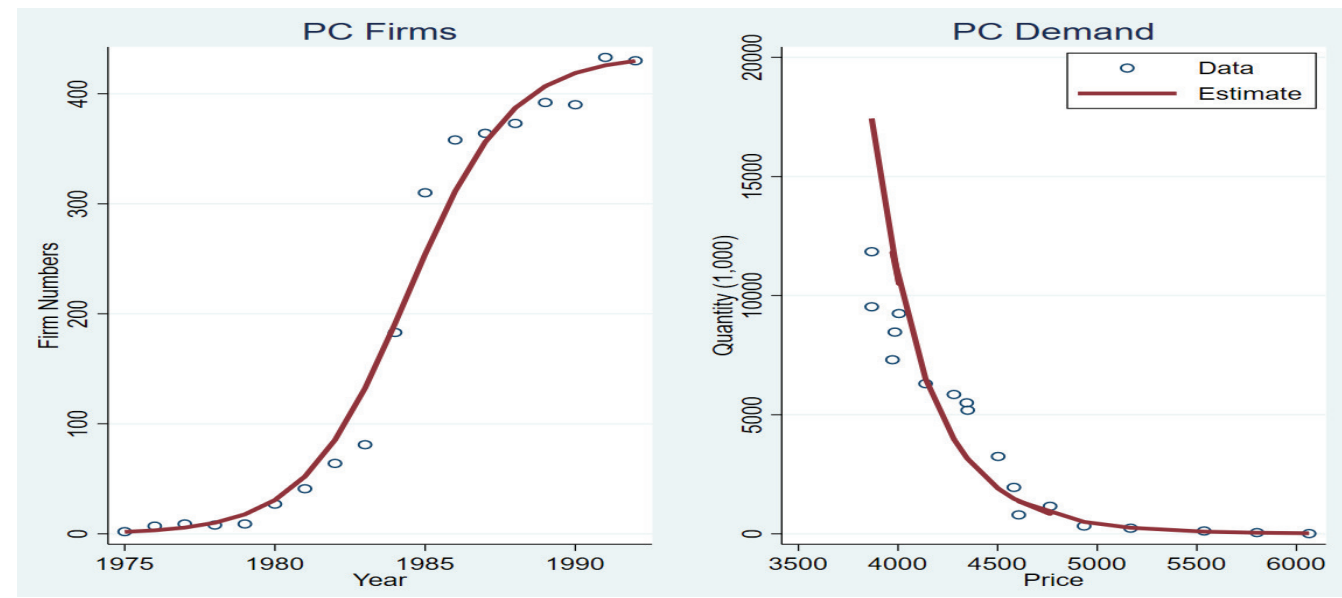

Fig. 10. PC Diffusion and Demand Estimates

\subsection{Model calibration}

To calibrate the model, we first pick values for $N, \gamma N$ and $k_{0}$ from the diffusion estimation for the auto and the PC industries, respectively. We then pick values for $\beta$ and $A$ from the demand estimation. Note that in the model, a firm's output is normalized to 1 per period. While this does not affect the theoretical analysis, we account for a firm's production size in the empirical applications. In doing so, we denote $q$ a firm's output and $Q$ the industry output, so $Q_{t}=q k_{t}$ at date $t$. Accordingly, we revise Eqs. (13), (14) and (24) as follows by replacing $A$ with $\tilde{A} q^{1-\beta}$ (where $\tilde{A}$ and $\beta$ are from the demand function estimation above):

$$
\begin{gathered}
\text { Regime 1: } \frac{1}{N-k_{0}} \int_{0}^{\infty} e^{-r t}\left(\alpha \frac{N}{k_{0}}+(1-\alpha) \frac{N}{k_{t}}-1\right) \tilde{A} q^{1-\beta} k_{t}^{1-\beta} d t=c ; \\
\text { Regime 2: } \frac{1}{N-k_{0}} \int_{0}^{\infty} e^{-r t}\left(\left(\frac{N}{k_{0}}\right)^{\alpha}\left(\frac{N}{k_{t}}\right)^{1-\alpha}-1\right) \tilde{A} q^{1-\beta} k_{t}^{1-\beta} d t=c ; \\
\text { Social optimum: } \quad \int_{0}^{\infty} e^{-(r+\gamma N) t}\left(\frac{k_{t}}{k_{0}}\right)^{2} \tilde{A} q^{1-\beta} k_{t}^{-\beta} d t=c .
\end{gathered}
$$

In the auto case, a firm on average produced less than 1,000 cars a year up to 1910, and we calibrate $q=900$ based on output per firm in 1910 and $\tilde{A} q^{1-\beta}=61.28$ 
(million). In the PC case, using output per firm in 1992, we calibrate $q=27,500$ and $\tilde{A} q^{1-\beta}=163.63$ (million).

We then set $r=0.05$. The two remaining parameters are $\alpha$ and $c$. Because we have no direct information about them, we assume $\alpha=0$ to pin down $c$ in the benchmark analysis. Since Regimes 1 and 2 coincide when $\alpha=0$, one can use either Eq. (38) or Eq. (39) to solve for $c$. Table 1 summarizes the benchmark parameter values calibrated for the auto and the PC industries. Because the values of $\alpha, r$ and $N$ are chosen by assumption, we will consider alternative values for them in Section 5.4 for robustness checks.

Table 1. Model Parameterization

\begin{tabular}{lccccccc}
\hline \hline & $\alpha$ & $r$ & $N$ & $\gamma N$ & $k_{0}$ & $\beta$ & $\tilde{A} q^{1-\beta}$ \\
\hline Auto & 0 & 0.05 & 210 & 0.53 & 3.31 & 0.28 & 61.28 \\
\hline PC & 0 & 0.05 & 435 & 0.58 & 1.78 & 0.07 & 163.63 \\
\hline \hline
\end{tabular}

Figure 11 plots the calibrated model dynamics for the auto industry. The number of firms $k_{t}$ grows along a logistic curve. Meanwhile, $v_{t}$ decreases while $u_{t}$ increases over time. ${ }^{19}$ The initial difference $v_{0}-u_{0}$ equals the innovation cost $c_{\text {Auto }}=\$ 173.73$ million (in 2012 price). By 1910, the value of a producer $v_{t}$ comes down to $\$ 274$ million and the value of a future imitator rises to $\$ 250$ million. Because almost all the potential entrants $N$ have entered the industry by then, the total value of firms $v_{1910} k_{1910}$ is very close to the present value of the industry revenue $p_{1910} Q_{1910} / r$.
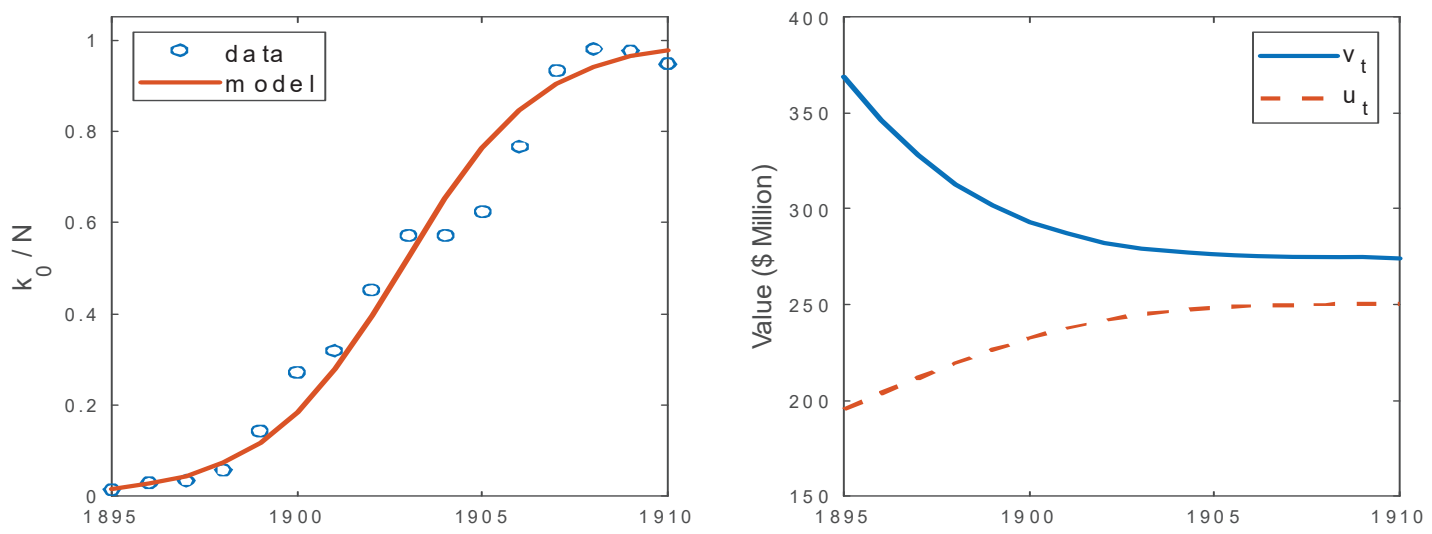

Fig. 11. Model Calibration: Auto

\footnotetext{
${ }^{19}$ Because we assume $\alpha=0$ in the benchmark calibration, Regimes 1 and 2 coincide. The modelimplied time paths of $v_{t}$ and $u_{t}$ are consistent with the proof in Appendix A.1, which shows that $v_{t}$ decreases in $t$ when $\alpha=0$ and $u_{t}$ increases in $t$ when $\beta<1$. More broadly, for $\alpha \in(0,1)$, Regimes 1 and 2 do not coincide and the time paths of $v_{t}$ and $u_{t}$ may look differently between the two regimes. For example, with certain parameter values, $v_{t}$ in Regime 1 may initially increase and later decrease in $t$, but $v_{t}$ in Regime 2 always decreases in $t$. Regardless, no innovator would enter after date 0 in either regime because the entry value of an innovator minus his option value of waiting to imitate always decreases in $t$, as shown in the proofs in Appendix A.1 and A.2.
} 
Figure 12 plots the calibration results for the PC industry. Again, the number of firms $k_{t}$ grows along a logistic curve, and $v_{t}$ decreases while $u_{t}$ increases over time. The initial difference $v_{0}-u_{0}$ equals the innovation cost $c_{\mathrm{PC}}=\$ 986.87$ million (in 2012 price). By 1992, the value of a producer $v_{t}$ comes down to $\$ 2.14$ billion and the value of a future imitator rises to $\$ 1.97$ billion. Because almost all the potential entrants have entered the industry by then, the total value of firms $v_{1992} k_{1992}$ is very close to the present value of the industry revenue $p_{1992} Q_{1992} / r$.
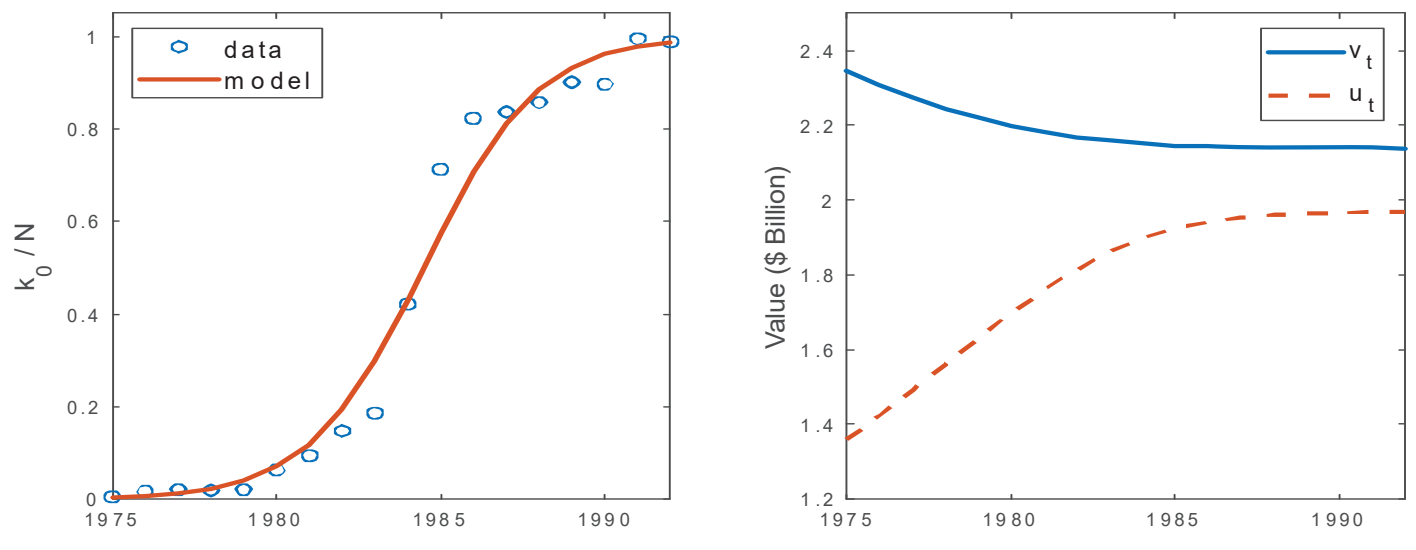

Fig. 12. Model Calibration: PC

\subsection{Counterfactual analysis}

Given the calibrated model parameter values, we then conduct counterfactual analysis and evaluate welfare.

\subsubsection{Optimal compensation for idea sellers}

We first evaluate the effect of the compensation share $\alpha$ in Regimes 1 and 2, and start with the auto industry. Given the innovation cost $c_{\text {Auto }}$ derived from the model calibration, we solve the equilibrium industry dynamics for each counterfactual value of $\alpha \in(0,1]$. Particularly, Eqs. (38) and (39) allow us to pin down the counterfactual entry number of innovators $k_{0}$ at date 0 . Figure 13 shows that $k_{0}$ strictly increases with $\alpha$ for both Regimes 1 and 2 when $0<\alpha<0.61$ and Regime 1 has a higher value of $k_{0}$ than Regime 2 . For $\alpha \geq 0.61$, the values of $k_{0}$ in both regimes reaches the corner solution $k_{0}=N$. Equation (40) pins down the socially optimal entry number of innovators $k_{0}^{*} / N$ to be 0.151 , which can be achieved by choosing $\alpha_{\text {Auto }}^{\mathbf{I} *}=0.07$ in Regime 1 and $\alpha_{\text {Auto }}^{\mathbf{I I} *}=0.167$ in Regime 2. The social optimum yields a social surplus $W_{0, \text { Auto }}^{*}=\$ 64.45$ billion (in 2012 price). 

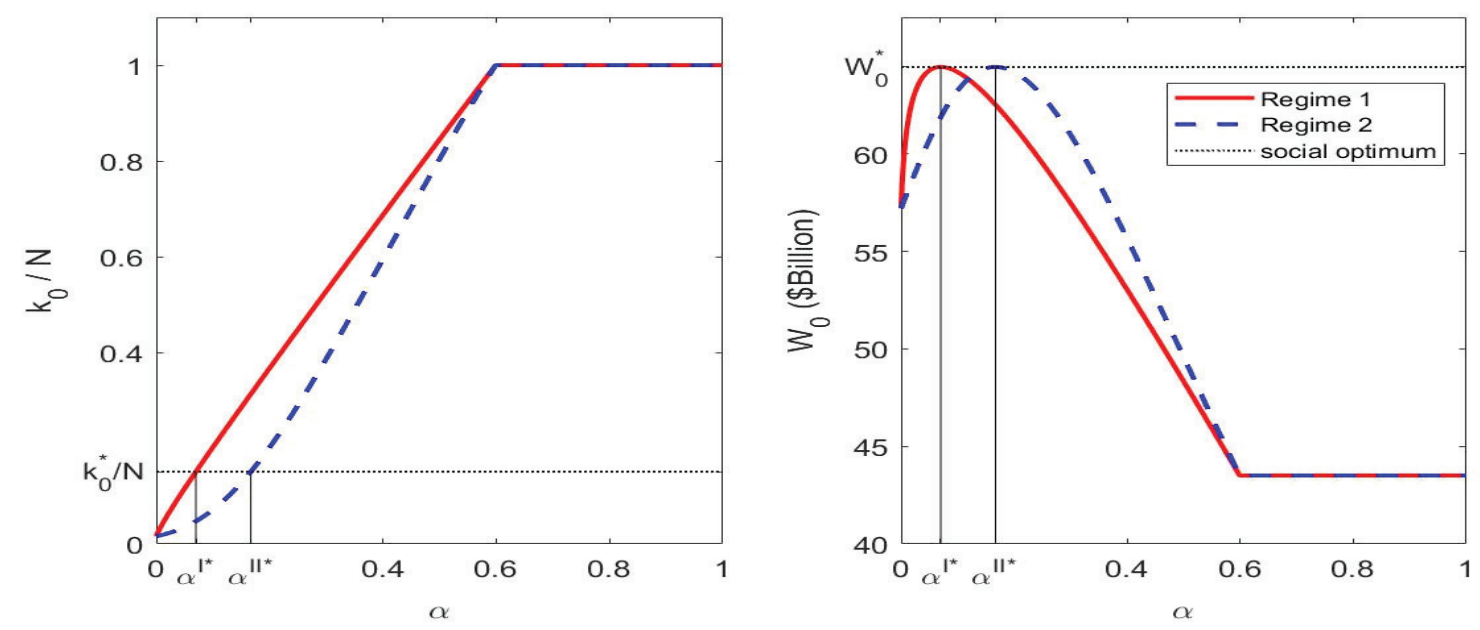

Fig. 13. Effect of $\alpha$ : Auto

We then look into the PC industry. Given the innovation $\operatorname{cost} c_{\mathrm{PC}}$ derived from the model calibration, Eqs. (38) and (39) pin down the entry number of innovators $k_{0}$ for each counterfactual value of $\alpha \in(0,1]$. Figure 14 shows that $k_{0}$ strictly increases with $\alpha$ for both Regimes 1 and 2 when $0<\alpha<0.42$ and Regime 1 has a higher value of $k_{0}$ than Regime 2 . For $\alpha \geq 0.42$, the values of $k_{0}$ in both regimes reaches the corner solution $k_{0}=N$. The socially optimal entry number of innovators $k_{0}^{*} / N$ is 0.164 , which can be achieved by choosing $\alpha_{\mathrm{PC}}^{\mathbf{I} *}=0.055$ in Regime 1 and $\alpha_{\mathrm{PC}}^{\text {II } *}=0.135$ in Regime 2. The social optimum yields a social surplus $W_{0, \mathrm{PC}}^{*}=\$ 798.9$ billion (in 2012 price).
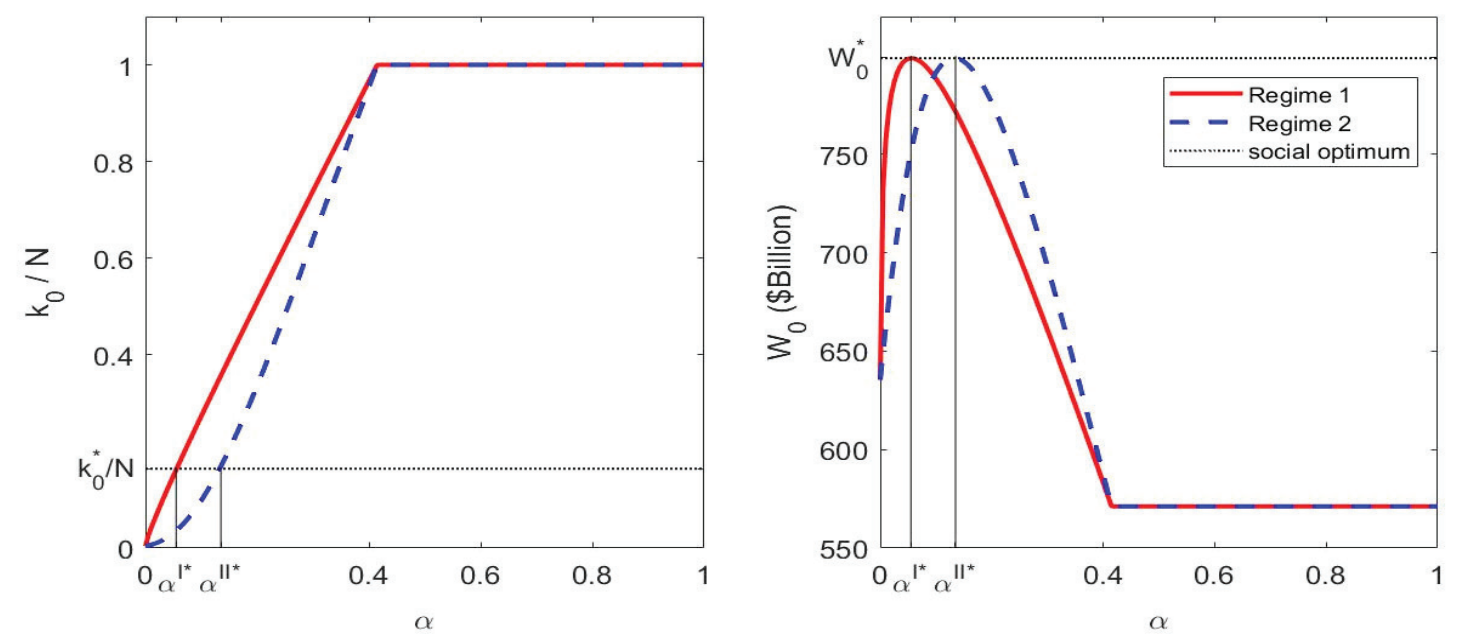

Fig. 14. Effect of $\alpha$ : PC

Comparative statics for $\alpha^{*}$. - Figure 15 plots comparative statics for the socially optimal compensation share $\alpha^{*}$ under Regimes 1 and 2 based on the auto calibration. The results show the following: 
- $\alpha^{*}$ increases with $\beta$.-A higher $\beta$ means a lower price elasticity, which leads price to decline faster which discourages $k_{0}$. This makes the congestion externality less of a concern, so $\alpha^{*}$ rises.

- $\alpha^{*}$ decreases with $\gamma$ (holding $N$ fixed, when $\gamma$ is sufficiently large).-A higher $\gamma$ implies a better imitation technology, so the planner would need less innovation when $\gamma$ is sufficiently large and so $\alpha^{*}$ falls.

- $\alpha^{*}$ rises with $c$ but falls with $\bar{A}\left(\equiv \tilde{A} q^{1-\beta}\right)$. - A higher $c$ or a lower $\bar{A}$ discourages $k_{0}$. This makes the congestion externality less of a concern, so $\alpha^{*}$ rises.

- $\alpha^{*}$ rises with $N$ (holding $\gamma N=\lambda$ fixed). - A higher $N$ leads to faster price decline which discourages $k_{0}$. This, together with a larger pool of potential adopters $N$, makes the congestion externality less of a concern, so $\alpha^{*}$ rises.

- Comparison of Regimes 1 and 2.- $\alpha^{*}$ is higher under Regime 2 than under Regime 1, and the difference rises with $\beta, \gamma, c / \bar{A}$, and $N$.
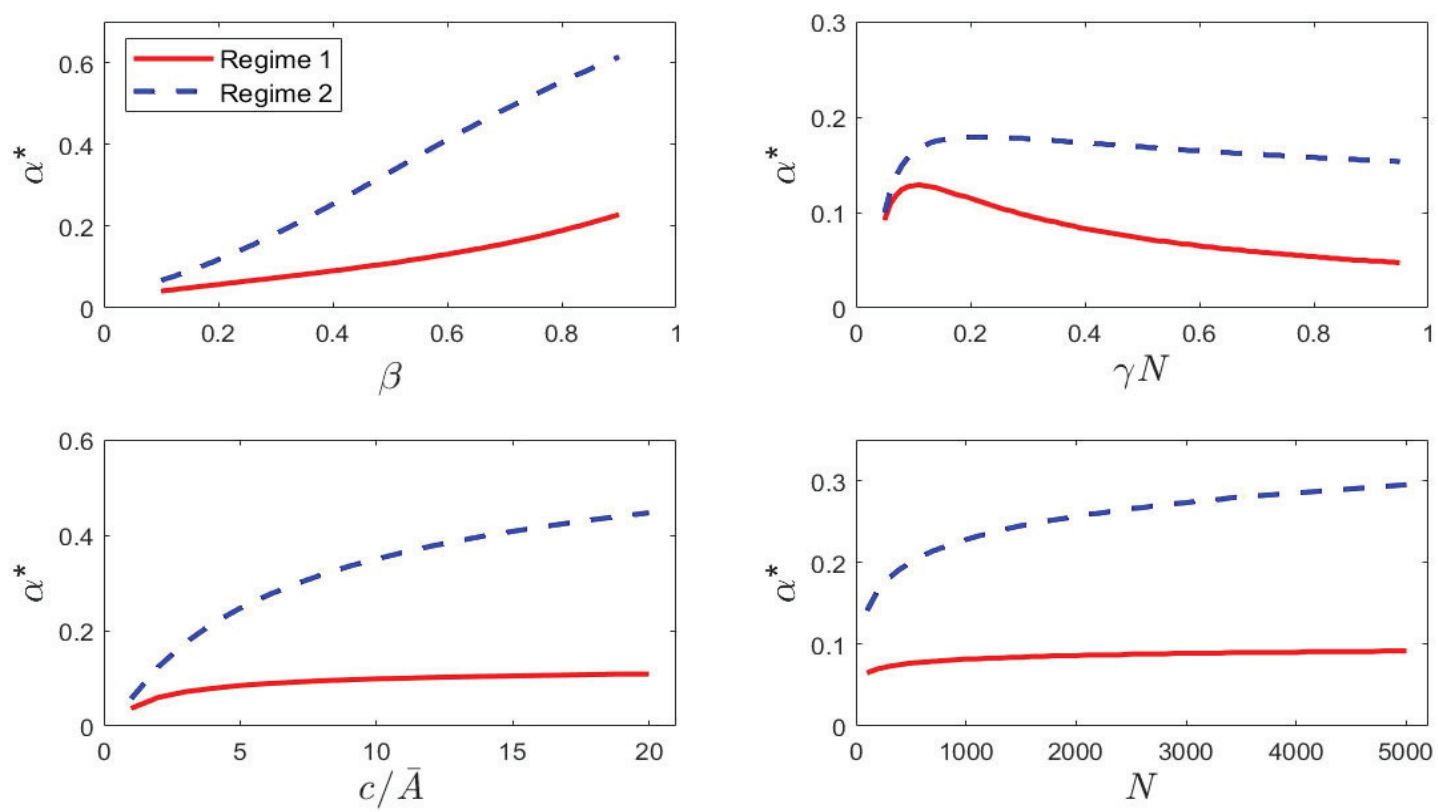

Fig. 15. Comparative Statics for $\alpha^{*}$ under Regimes 1 And 2

The comparative statics help explain the difference in $\alpha^{*}$ between the auto and the $\mathrm{PC}$ industries. Compared to the auto, the $\mathrm{PC}$ industry has a smaller $\beta$ and a larger $\gamma N$, and these two dominate the offsetting forces of the larger $c / \bar{A}$ and larger $N$ and hence $\alpha_{\mathrm{PC}}^{*}<\alpha_{\mathrm{Auto}}^{*}$ under each regime. Quantitatively, by comparing counterfactuals that let one industry take on the other industry's parameter values, we find that the smaller $\beta$ (i.e., the higher price elasticity) accounts most for the smaller $\alpha_{\mathrm{PC}}^{*}$. 


\subsubsection{Optimal innovation subsidy}

Given $\alpha=0$, the entry of innovators is lower than the socially optimal level. Providing innovators a subsidy $s$, instead of setting a socially optimal $\alpha^{*}$, can also help achieve the social optimality.

Note that with the subsidy, $c-s$ is the net entry cost for innovators. Figure 16 plots the effect of $s$ on the entry of innovators $k_{0}$ and welfare $W_{0}$. The results show that $k_{0}$ increases with $s$, and the social welfare peaks at $s^{*} / c=0.61$ for the auto industry and $s^{*} / c=0.62$ for the PC industry.
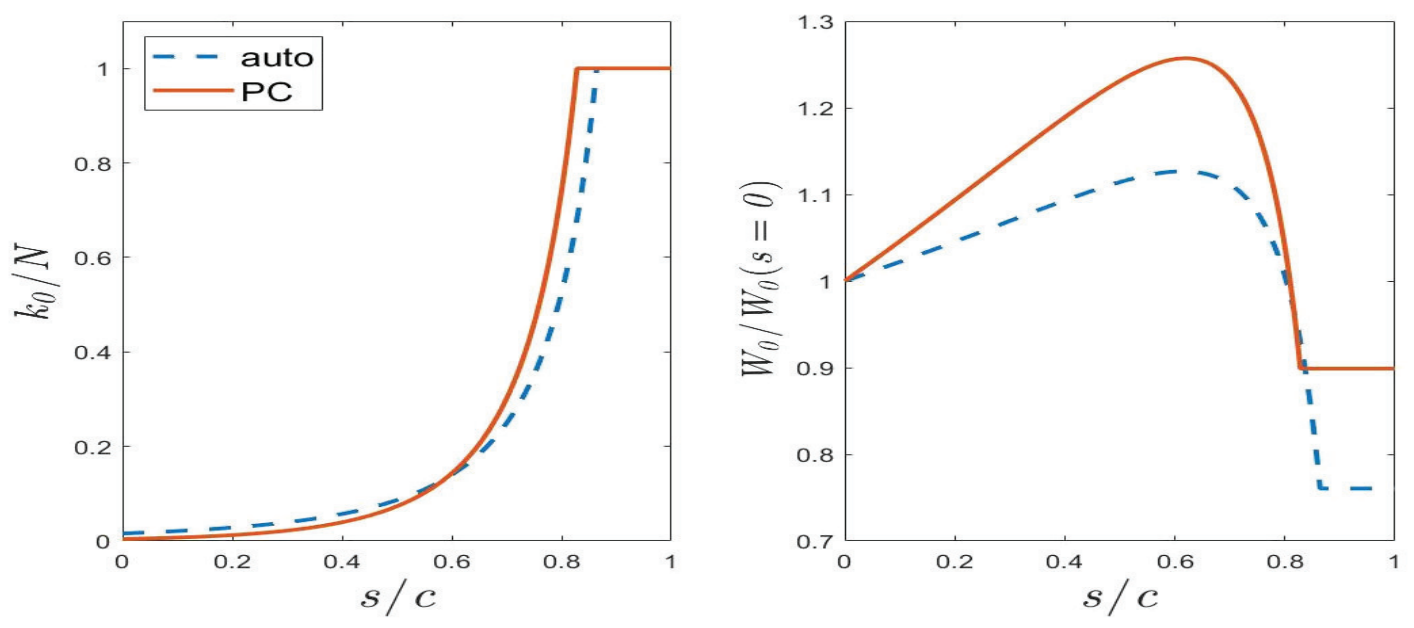

Fig. 16. Effect of the Subsidy $s$

\subsubsection{Optimal diffusion rate}

We can similarly evaluate the effects of varying the diffusion rate $\gamma$ (holding $N$ fixed). Consider again the scenario where incumbents are not compensated by imitators, so $\alpha=0$. Should the planner slow down the diffusion?
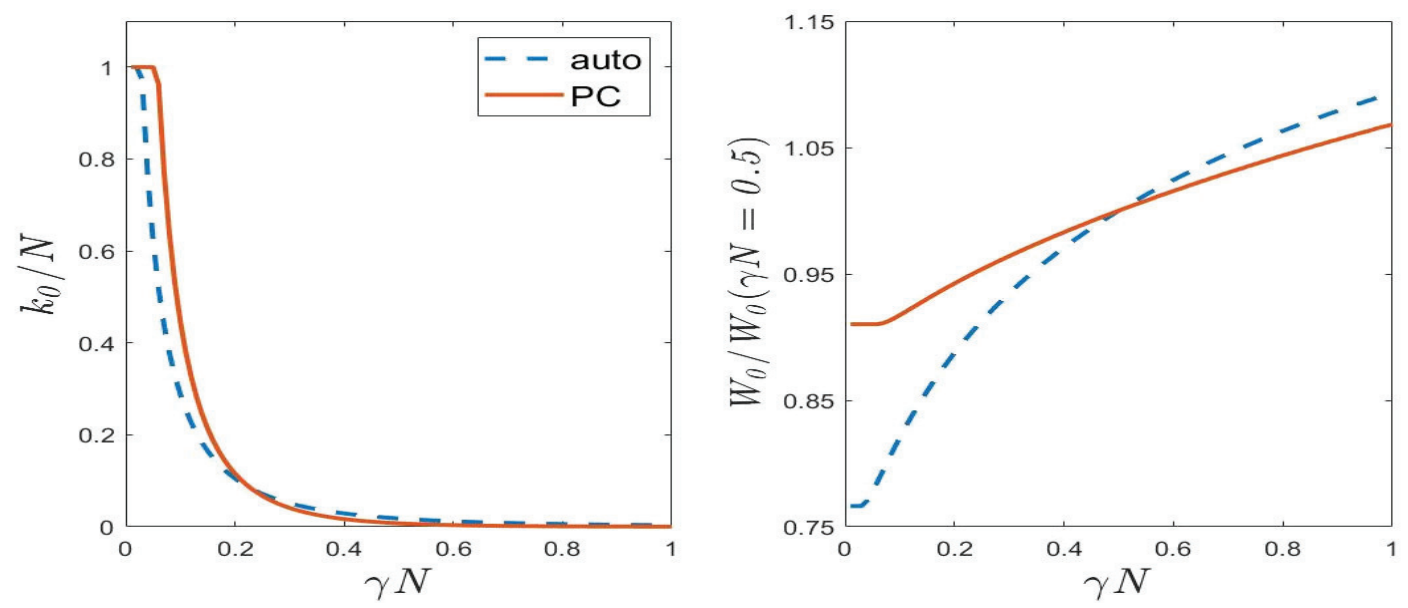

Fig. 17. Effect of the Diffusion Rate $\gamma$ 
Figure 17 shows that for both the auto and the PC industries, $k_{0}$ decreases with $\gamma$ while $W_{0}$ increases with $\gamma$. Therefore, if the planner were to push down $\gamma$, the entry of innovators $k_{0}$ would increase but social welfare would decline. The intuition is that while slowing down diffusion could encourage entry of innovators, it would forego too much free learning and the welfare effect of the latter dominates.

\subsection{Robustness checks}

For robustness checks, we redo the above exercises with alternative assumptions on $N, r$ and $\alpha$. The results are consistent with our previous findings.

\subsubsection{Pool of potential entrants}

In the benchmark analysis, we assumed that the shakeout started after almost all the potential firms had entered the industry. Alternatively, one could consider that the shakeout started in the middle of the diffusion process, so there might be a larger pool of potential entrants. For example, we may assume $N=1,000$ (instead of 210) for the auto case and $N=2,000$ (instead of 425) for the PC case. In each case, we then obtain a smaller $\gamma N$ from the diffusion estimation. The new estimates imply that it would take 30 years for each industry to reach $99 \%$ adoption rate among potential producers had the shakeout not happened, doubling what is assumed in the benchmark calibration.

We then re-do the calibration and counterfactual exercises with the alternative $N$. Regarding the socially optimal compensation for idea sellers, we now find for the auto case, $\alpha_{\text {Auto }}^{\mathbf{I} *}=0.134$ under Regime 1 or $\alpha_{\text {Auto }}^{\mathbf{I I} *}=0.309$ under Regime 2, while for the PC case, $\alpha_{\mathrm{PC}}^{\mathbf{I} *}=0.095$ under Regime 1 or $\alpha_{\mathrm{PC}}^{\mathbf{I I} *}=0.215$ under Regime 2 . These estimates of $\alpha^{*}$ are larger than those found in the benchmark analysis, due to the larger $N$ and smaller $\gamma N$ and higher $c$ from the re-calibrated models, which is consistent with the prediction of our comparative-statics analysis (cf. Fig. 15). We also find that the social optimum can be achieved by subsidizing 65.7 percent of the innovation cost in the auto case, or 62.1 percent in the PC case.

\subsubsection{Anticipated shakeout}

Our model can also be extended to allow the shakeout being anticipated. Specifically, we could assume that the industry expects a disruptive innovation to arrive at a Poisson rate $\rho$. This innovation would make obsolete existing technologies and drive firm values to zero. ${ }^{20}$

\footnotetext{
${ }^{20}$ For example, an industry may expects a disruptive innovation (e.g., the assembly line in the auto case) to arrive at a Poisson rate $\rho$. This innovation would require an incumbent firm to incur a big capital investment to produce a newly designed product at a large scale. When that innovation does arrive, the new (and lower) equilibrium price can only support the capital investment made by a few firms and the rest have to exit. As a result, the present value of an investing firm (net of its
} 
Accordingly, the value of an incumbent firm under Regime 2 satisfies

$$
r v_{t}=p_{t}+\gamma\left(N-k_{t}\right) \alpha v_{t}-\rho v_{t}+\frac{d v_{t}}{d t}
$$

i.e.,

$$
(r+\rho) v_{t}=p_{t}+\gamma\left(N-k_{t}\right) \alpha v_{t}+\frac{d v_{t}}{d t}
$$

Note that $\rho>0$ in Eq. (41) is equivalent to raising $r$ to $r+\rho$ in Eq. (10). Similarly, we can revise the value function conditions for outsiders as well as for Regime 1 and for the social planner's problem. The original functional forms of our model hold, except that $r$ becomes $r+\rho$.

Considering that the shakeout occurred in the 16th year for the auto industry and in the 18th year for the PC industry, we take the average and calibrate $\rho=1 / 17=$ 0.06. Accordingly, we set $r+\rho=0.05+0.06=0.11$ and redo the model calibration and counterfactual analysis.

Regarding the socially optimal compensation for idea sellers, we now find in the auto case, $\alpha_{\text {Auto }}^{\mathbf{I} *}=0.147$ under Regime 1 or $\alpha_{\text {Auto }}^{\mathbf{I I} *}=0.296$ under Regime 2, while in the PC case, $\alpha_{\mathrm{PC}}^{\mathbf{I} *}=0.115$ under Regime 1 or $\alpha_{\mathrm{PC}}^{\mathbf{I I} *}=0.235$ under Regime 2 . The values of $\alpha^{*}$ are larger than the benchmark analysis due to the higher discount $r+\rho$ in spite of lower $c$ implied by the re-calibrated models. We also find that the social optimum can be achieved by subsidizing 59.2 percent of the innovation cost in the auto case or 58.4 percent in the PC case.

\subsubsection{Idea sellers' bargaining share}

We assumed $\alpha=0$ in the benchmark model calibration. For robustness checks, we re-do the calibration for other values $\alpha \in(0,1]$. The results are plotted in Fig. 18 .
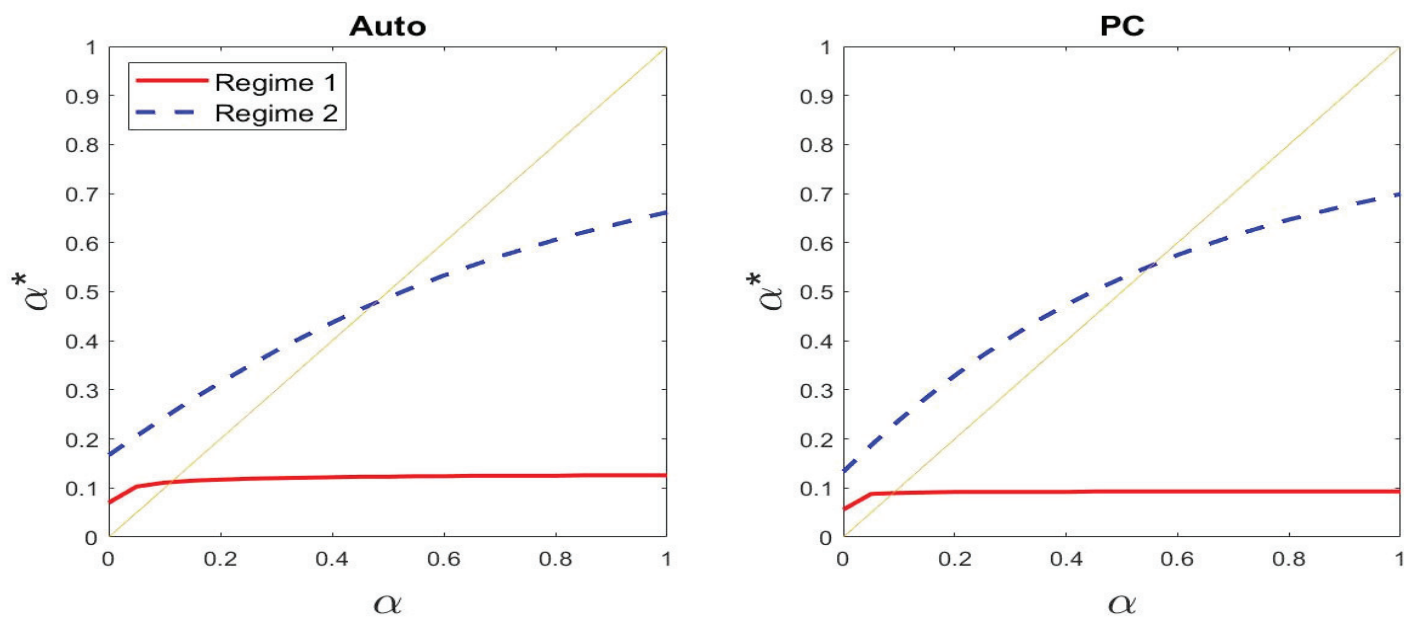

Fig. 18. Assumed $\alpha$ And Implied $\alpha^{*}$

investment costs) is zero, and the value of an exiting firm is also zero. 
Note that the larger value $\alpha$ we assume in the calibration, the higher innovation cost $c$ must be to rationalize the observations. But then, as Fig. 18 shows, the implied rise in $c$ means that $\alpha^{*}$ rises, consistent with our comparative-statics analysis shown in Fig. 15.

Moreover, Fig. 18 shows that $\alpha^{*} \in(0,1)$ and that it crosses the 45 -degree line. In the range where $\alpha^{*}>\alpha$, an innovation subsidy could help improve welfare; otherwise, a tax would do so.

Finally, for all $\alpha \in[0,1], \alpha^{*}$ remains small in Regime 1 between $7 \%-12.6 \%$ for the auto and between 5.6\%-9.3\% for the PC. In Regime 2, as the assumed $\alpha$ gets larger, $\alpha^{*}$ does increase quite a bit for both industries and the optimal $\alpha^{*}$ for the PC eventually exceeds that for the auto as the effect of $c$ starts to dominate. However, to the extent that Regime 2 applies naturally to scenarios of non-patented know-how, a small value of assumed $\alpha$ is more realistic, which would also imply a small $\alpha^{*}$. Note that both autos and PCs appear to have highly elastic demand curves - we estimate $\beta_{\mathrm{PC}}$ and $\beta_{\text {Auto }}$ to be quite small. Imitator entry then drives prices down slowly, and that encourages innovation, and raises the congestion externality that innovators face. Then $\alpha_{\text {Auto }}^{*}$ and $\alpha_{\mathrm{PC}}^{*}$ should both be low, especially $\alpha_{\mathrm{PC}}^{*}$, because PC demand is more price elastic.

\section{$6 \quad$ The $N \rightarrow \infty$ limit}

In this section, we study a limiting version of our model as $N \rightarrow \infty$ which yields a constant growth of firm numbers. Because the limiting model does not incorporate the congestion externality in the diffusion process, it implies that letting initial innovators extract the entire rents (i.e., $\alpha=1$ ) is socially optimal.

The special case where $N \rightarrow \infty$ does not fit the industry data well, but we present it here because of its simplicity and because it relates to earlier models of competitive innovation: Boldrin and Levine (2008) and Quah (2002) assume a simple diffusion process

$$
\frac{d k_{t}}{d t}=\lambda k_{t}
$$

so that

$$
k_{t}=k_{0} e^{\lambda t}
$$

The following proposition connects our model with theirs. We shall now characterize the limiting model by letting $N$ get large while at the same time reducing $\gamma$ so that the logistic diffusion process (3) converges to the one in Eq. (42):

Proposition 12 Let $\gamma \rightarrow 0$ and $N \rightarrow \infty$ in such a way that $\gamma N \rightarrow \lambda>0$, a constant. The incumbents' meeting rate then converges to a constant: $\left.\frac{d k_{t} / d t}{k_{t}}\right|_{N \rightarrow \infty} \rightarrow \lambda$ for all $t \geq 0$. 
Proof. See Appendix A.12.

Therefore, a constant growth of firm numbers can be seen as a limiting version of the logistic diffusion studied in our model as $N \rightarrow \infty$. In what follows we shall assume that

$$
\lambda<r
$$

to ensure that social welfare derived from the innovation is bounded.

\subsection{Equilibrium when $N \rightarrow \infty$}

The following propositions characterize the equilibria for the limiting model.

Proposition 13 If condition (44) holds, in Regime 1 an innovator's value at date $t$ is

$$
v_{t}=\frac{A k_{0}^{-\beta} e^{-\beta \lambda t}}{r+\beta \lambda}+\frac{\alpha \lambda A k_{0}^{-\beta}}{(r+\beta \lambda)(r+(\beta-1) \lambda)} e^{-(\beta-1) \lambda t},
$$

an imitator's value is

$$
\omega_{t}=\frac{A k_{0}^{-\beta}}{r+\beta \lambda} e^{-\beta \lambda t}
$$

and an outsider's value is

$$
u_{t}=0 \text {. }
$$

Entry of innovators at date 0 is

$$
k_{0}^{\mathbf{I}}=\left(\frac{A(r+(\beta-1) \lambda+\alpha \lambda)}{c(r+\beta \lambda)(r+(\beta-1) \lambda)}\right)^{\frac{1}{\beta}},
$$

which is valid for $\beta \geq 1$ or for all $\beta>0$ if $\alpha=0$. No innovator enters after date 0 .

Proof. See Appendix A.13.

Proposition 14 If condition (44) holds, in Regime 2 the value of a producer (innovator or imitator) at date $t$ is

$$
v_{t}=\frac{A k_{0}^{-\beta} e^{-\beta \lambda t}}{r+(\beta-\alpha) \lambda}
$$

and an outsider's value is

$$
u_{t}=0 \text {. }
$$

Entry of innovators at date 0 is

$$
k_{0}^{\mathbf{I I}}=\left(\frac{A}{c(r+(\beta-\alpha) \lambda)}\right)^{\frac{1}{\beta}},
$$

which is valid for all $\beta>0$. No innovator enters after date 0 . 
Proof. See Appendix A.14.

Comparing the two regimes, we have the following findings.

\section{Proposition 15}

$$
\text { (A) } k_{0}^{\mathbf{I}} \text { and } k_{0}^{\mathbf{I I}}\left\{\begin{array}{l}
\text { increase with } \alpha \text { and } A, \\
\text { decrease with } c \text { and } r, \\
\text { decreases with } \lambda \text { if } \beta>\alpha .
\end{array}\right.
$$

(B) All parameters being equal across the two regimes,

$$
\frac{k_{0}^{\mathbf{I}}}{k_{0}^{\mathbf{I I}}}=\left\{\begin{array}{lr}
1 & \text { for } \alpha \in\{0,1\} \\
>1 & \text { for } \alpha \in(0,1)
\end{array} .\right.
$$

Proof. See Appendix A.15.

Proposition 15 shows that the limiting model yields comparative statics for $k_{0}$ consistent with our previous findings. However, the welfare implications can be quite different, as shown in the following.

\subsection{Welfare analysis when $N \rightarrow \infty$}

Optimal compensation for idea sellers The limiting model implies that allowing the original innovators to extract the entire rents from succeeding imitators would yield the socially optimal incentive for innovation. This result holds for both Regimes 1 and 2. Formally, we assume that condition (44) holds and prove the following result:

Proposition 16 (A) It is socially optimal to innovate only at date 0. (B) The socially optimal number of innovators is

$$
k_{0}^{*}=\left(\frac{A}{(r+(\beta-1) \lambda) c}\right)^{\frac{1}{\beta}} .
$$

(C) Social optimum implies $\alpha^{*}=1$ for both Regimes 1 and 2.

Proof. See Appendix A.16.

Why does the limiting model yield an optimal compensation share for idea sellers different from our previous finding? The key is that in the model with logistic diffusion there is a congestion externality that an innovator creates and ignores - He reduces the meeting rate for other innovators. However, in the limiting model, the meeting rate for an innovator is fixed ( cf. $\frac{d k_{t} / d t}{k_{t}}=\lambda$ ). Therefore, by considering a more realistic logistic diffusion process, we not only fit the industry evolution pattern better but also uncover novel implications of congestion externalities that take place during the idea diffusion process. 
Optimal innovation subsidy Given that the optimal compensation for idea sellers is $\alpha^{*}=1$, the planner could offer an innovation subsidy $s^{*}>0$ to achieve the social optimum whenever $\alpha<1$. With our solutions for $k_{0}^{\mathbf{I}}, k_{0}^{\mathbf{I I}}$ and $k_{0}^{*}$ (cf. Propositions 13, 14 and 16), we prove the following result:

Proposition 17 Social optimum implies $0<s^{\mathbf{I} *}<s^{\mathbf{I} * *}$ for $\alpha \in(0,1), s^{\mathbf{I} *}=s^{\mathbf{I I} *}=$ $\frac{c \lambda}{r+\beta \lambda}>0$ for $\alpha=0$, and $s^{\mathbf{I} *}=s^{\mathbf{I I} *}=0$ for $\alpha=1$.

Proof. See Appendix A.17.

Note that the socially optimal subsidy never goes negative (i.e., becomes a tax), which is in contrast to our finding with logistic diffusion. Again, this is because the limiting model incorporates only knowledge spillovers but not the meeting congestion.

Optimal diffusion rate Parallel to the finite- $N$ case where we showed that the planner does not want to reduce $\gamma$, here the planner does not want to reduce $\lambda$, as shown in the following claim:

Proposition 18 For any values of $\alpha$ and $\beta$ permitted by the limiting model, social welfare increases with $\lambda$ for both Regimes 1 and 2.

Proof. See Appendix A.18.

\section{Conclusion}

We modeled an innovation and its diffusion in one industry and discussed policy and welfare. Capacity constraints imply that licensing raises the revenues of innovators and that licensing is also socially beneficial to a degree. We showed that the welfare outcome depends on whether imitators can resell the innovation, and on how much the innovators are compensated for transferring the innovation.

The socially optimal bargain allocation hinges on the diffusion process, particularly the congestion externality in meetings between innovators and imitators. Our analysis also showed that slowing down diffusion encourages innovation and raises initial capacity, but that it lowers imitation so that capacity grows more slowly. We argued that this may help explain why Silicon Valley overtook Route 128.

We calibrated the model to data for the U.S. automobile and personal computer industries. Though starting nearly one century apart, the industries shared the basic feature of an $S$-shaped diffusion prior to the shakeout. Our empirical findings match well the expansion of firm numbers prior to the shakeout in each industry and quantify the theoretical predictions of the model. 


\section{References}

[1] Atkeson, A. (2020) "What will be the economic impact of Covid-19 in the US? Rough estimates of disease scenarios." NBER Working Paper 26867.

[2] Bass, F. (1969). "A new product growth for model consumer durables." Management Science, 15(5), 215-227.

[3] Bass, F. (2004). "Comments on "A new product growth for model consumer durables": The Bass model." Management Science, 50(12), 1833-1840.

[4] Benhabib, J., Perla, J., \& Tonetti, C. (2021). "Reconciling models of diffusion and innovation: A theory of the productivity distribution and technology frontier." Econometrica, 89(5), 2261-2301.

[5] Bishara, N. D. (2011). "Fifty ways to leave your employer: Relative enforcement of covenants not to compete, trends, and implications for employee mobility policy." University of Pennsylvania Journal of Business Law, 13, 751-795.

[6] Boldrin, M., \& Levine, D. K. (2008). "Perfectly competitive innovation." Journal of Monetary Economics, 55(3), 435-453.

[7] Burdett, K., \& Coles, M. G. (2001). "Transplants and implants: The economics of self-improvement." International Economic Review, 42(3), 597-616.

[8] Cabral, L., Wang, Z., \& Xu, D. Y. (2018). "Competitors, complementors, parents and places: Explaining regional agglomeration in the U.S. Auto Industry." Review of Economic Dynamics, 30, 1-29.

[9] Chade, H., Eeckhout, J., \& Smith, L. (2017). "Sorting through search and matching models in economics." Journal of Economic Literature, 55(2), 493-544.

[10] David, P. A. (1969). "A contribution to the theory of diffusion." Stanford University Research Center in Economic Growth Memorandum 71.

[11] Filson, D. (2001). "The nature and effects of technological change over the industry life cycle." Review of Economic Dynamics, 4(2), 460-494.

[12] Franco, A. M., \& Mitchell, M. F. (2008). "Covenants not to compete, labor mobility, and industry dynamics." Journal of Economics $\&$ Management Strategy, $17(3), 581-606$.

[13] Garibaldi, P., Moen, E. R., \& Pissarides, C. A. (2020). "Modelling contacts and transitions in the SIR epidemics model." Covid Economics, Vetted and RealTime Papers, 5, 1-20. 
[14] Gilson, R. J. (1999). "The legal infrastructure of high technology industrial districts: Silicon Valley, Route 128, and Covenants Not to Compete." New York University Law Review, 74, 575-629.

[15] Gort, M., \& Klepper, S. (1982). "Time paths in the diffusion of product innovations." Economic Journal, 92, 630-653.

[16] Griliches, Z. (1957). "Hybrid corn: An exploration in the economics of technical change." Econometrica 25(4), 501-522.

[17] Hayashi, F., Li, B. G., \& Wang, Z. (2017). "Innovation, deregulation, and the life cycle of a financial service industry." Review of Economic Dynamics, 26, 180-203.

[18] Hopenhayn, H., \& Shi, L. (2020). "Knowledge creation and diffusion with limited appropriation." Working Paper.

[19] Hosios, A. J. (1990). "On the efficiency of matching and related models of search and unemployment." Review of Economic Studies, 57(2), 279-298.

[20] Information Technology Industry Data Book. (1998). Washington, DC: Information Technology Industry Council.

[21] Jovanovic, B., \& MacDonald, G. (1994). "The life cycle of a competitive industry." Journal of Political Economy, 102 (2), 322-347.

[22] Klepper, S. (1996). "Entry, exit, growth, and innovation over the product life cycle." American Economic Review, 86, 562-583.

[23] Klepper, S. (2010). "The origin and growth of industry clusters: The making of Silicon Valley and Detroit." Journal of Urban Economics, 67(1), 15-32.

[24] Kydland, F. E., \& Prescott, E. C. (1977). "Rules rather than discretion: The inconsistency of optimal plans." Journal of Political Economy, 85(3), 473-492.

[25] Lauermann, S., Nöldeke, G., \& Tröger, T. (2020). "The balance condition in search-and-matching models." Econometrica, 88(2), 595-618.

[26] Manea, M. (2021). "Bottleneck links, essential intermediaries and competing paths of diffusion in networks." Theoretical Economics, 16(3), 1017-1053.

[27] Mansfield, E. (1961). "Technical change and the rate of imitation." Econometrica, 29(4), 741-766.

[28] Manuelli, R., \& Seshadri, A. (2014). "Frictionless Technology Diffusion: The Case of Tractors." American Economic Review, 104(4), 1368-91. 
[29] Nöldeke, G., \& Samuelson, L. (2015). "Investment and competitive matching." Econometrica, 83(3), 835-896.

[30] Quah, D. (2002). “24/7 Competitive innovation.” London School of Economics Centre for Economic Performance Working Paper 1218.

[31] Samila, S., \& Sorenson, O. (2011). "Noncompete covenants: Incentives to innovate or impediments to growth." Management Science, 57(3), 425-438.

[32] Saxenian, A. (1994). Regional advantage: Culture and competition in Silicon Valley and Route 128. Cambridge, MA: Harvard University Press.

[33] Shi, L. (2022). "Optimal regulation of noncompete contracts." Econometrica, forthcoming.

[34] Smith, P. H. (1970). Wheels within wheels: A short history of American motor car manufacturing. Funk and Wagnalls, New York.

[35] Stavins, J. (1995). "Model entry and exit in a differentiated-product industry: The personal computer market." Review of Economics and Statistics 77(4), 571584 .

[36] Thomas Register of American Manufacturers (1958-1999). Springfield, IL: Thomas.

[37] Thomas, R. P. (1977). An analysis of the pattern of growth of the automobile industry 1895-1929. Arno Press, New York.

[38] Utterback, J., \& Suarez, F. (1993). "Innovation, competition, and industry structure." Research Policy, 22, 1-21.

[39] Williamson, S. H. (2020). "What was the U.S. GDP then?" MeasuringWorth.

[40] Young, H. P. (2009). "Innovation diffusion in heterogeneous populations: Contagion, social influence, and social learning." American Economic Review, 99, 1899-1924. 


\title{
Appendix to "Idea Diffusion and Property Rights"
}

\author{
Boyan Jovanovic and Zhu Wang
}

\section{A. Proofs.}

\section{A.1. Proof of Proposition 1}

Proof. Solving the differential equations (7)-(9) directly is only feasible for special cases, so we develop an alternative approach to prove Proposition 1 . We then provide a special-case example for which we solve the differential equations (7)-(9) directly for a cross check.

(A) In Regime 1, imitators cannot resell ideas to other imitators. A potential adopter can copy an idea from an imitator but the fee goes to the idea's original innovator. We first assume that at equilibrium, innovators only enter at date 0 , so the time path of firm numbers is determined by Eq. (4) that

$$
k_{t}=\frac{N e^{\gamma N t}}{e^{\gamma N t}+\frac{N}{k_{0}}-1} .
$$

We then check if any agent would want to deviate by entering as an innovator at a date $\tau>0$.

The entry of a measure-zero innovator at $\tau>0$ would not change the industry quantity and price through Eq. (4). Upon entry, the value of this innovator is determined by two sources: One is that he will receive a fraction $1 / k_{t}$ of the total industry revenues $A k_{t}^{1-\beta}$ at each date $t \geq \tau$ by selling goods; the other is that he will get a chance $1 / k_{\tau}$ to collect idea-sale revenues from new imitators at each date $t \geq \tau$ (Note that $k_{\tau}$ is the number of incumbent firms, including both innovators and imitators, at his entry date $\tau$ ). At each date $t \geq \tau$, a fraction $\frac{k_{t}-k_{\tau}}{k_{t}}$ of firms in the industry are imitators who enter between date $\tau$ and date $t$, so this new innovator at his entry date $\tau$ expects to have $1 / k_{\tau}$ chance to receive the discounted sum of the fraction $\alpha\left(k_{t}-k_{\tau}\right) / k_{t}$ of the total industry revenues $A k_{t}^{1-\beta}$ as idea-sale revenues starting from date $\tau .^{21}$

Therefore, the value of a new innovator at his entry date $\tau$, denoted by $v_{\tau}^{\tau}$, can be written as

$$
v_{\tau}^{\tau}=\int_{\tau}^{\infty} e^{-r(t-\tau)}\left(\frac{1}{k_{t}}+\frac{\alpha}{k_{\tau}}\left(\frac{k_{t}-k_{\tau}}{k_{t}}\right)\right) A k_{t}^{1-\beta} d t .
$$

Note that the entry value of an innovator $v_{\tau}^{\tau}$ varies by entry date $\tau$ because the number of existing firms $k_{\tau}$ increases with $\tau$. Also, $v_{\tau}^{\tau}$ is different from the value of an

\footnotetext{
${ }^{21}$ In Regime 1, $\omega_{t}$ is an imitator's date- $t$ present value of revenues from selling goods. It is not feasible to characterize $v_{\tau}^{\tau}$ by calculating the integral of $\omega_{t}$ over time except for special cases (e.g., $\beta=1)$. Instead, we calculate the date- $\tau$ present value of imitators' shares of industry revenues.
} 
existing innovator at date $\tau$ who entered before $\tau$. In fact, an innovator who enters at date 0 should have the value at date $\tau$ :

$$
v_{\tau}^{0}=\int_{\tau}^{\infty} e^{-r(t-\tau)}\left(\frac{1}{k_{t}}+\frac{\alpha}{k_{0}}\left(\frac{k_{t}-k_{\tau}}{k_{t}}\right)\right) A k_{t}^{1-\beta} d t,
$$

So $v_{\tau}^{\tau}<v_{\tau}^{0}$ for any $\tau>0$, and $v_{\tau}^{\tau}=v_{\tau}^{0}$ for $\tau=0$.

Equation (4) implies that for any date $t \geq \tau$,

$$
k_{t}=\frac{N e^{\gamma N(t-\tau)}}{e^{\gamma N(t-\tau)}+\left(\frac{N}{k_{\tau}}-1\right)}
$$

and

$$
\frac{k_{t}}{k_{\tau}}=\frac{N e^{\gamma N(t-\tau)}}{N+\left(e^{\gamma N(t-\tau)}-1\right) k_{\tau}} .
$$

We can rewrite Eq. (48) as

$$
v_{\tau}^{\tau}=\int_{\tau}^{\infty} e^{-r(t-\tau)}\left(1-\alpha+\alpha \frac{k_{t}}{k_{\tau}}\right) A k_{t}^{-\beta} d t .
$$

Defining $s=t-\tau$, Eq. (52) becomes

$$
v_{\tau}^{\tau}=\int_{0}^{\infty} e^{-r s}\left(1-\alpha+\alpha \frac{k_{\tau+s}}{k_{\tau}}\right) A k_{\tau+s}^{-\beta} d s .
$$

Note that Eqs. (50) and (51) imply that

$$
\frac{k_{\tau+s}}{k_{\tau}}=\frac{N e^{\gamma N s}}{N+\left(e^{\gamma N s}-1\right) k_{\tau}}, \quad k_{\tau+s}^{-\beta}=\left(\frac{N e^{\gamma N s}}{e^{\gamma N s}+\left(\frac{N}{k_{\tau}}-1\right)}\right)^{-\beta},
$$

which both decrease in $k_{\tau}$. In Eq. (53), because $k_{\tau}$ increases in $\tau, \frac{k_{\tau+s}}{k_{\tau}}$ and $k_{\tau+s}^{-\beta}$ decrease in $\tau$, and hence $v_{\tau}^{\tau}$ decreases in $\tau$.

Similarly, because an imitator can keep $(1-\alpha)$ share of his output, the total value of outsiders $u_{\tau}\left(N-k_{\tau}\right)$ at date $\tau$ equals the imitators' share of the total discounted industry revenues from date $\tau$ and onward. Therefore, we have

$$
u_{\tau}\left(N-k_{\tau}\right)=\int_{\tau}^{\infty} e^{-r(t-\tau)}\left(\frac{(1-\alpha)\left(k_{t}-k_{\tau}\right)}{k_{t}}\right) A k_{t}^{1-\beta} d t,
$$

which implies

$$
\left.u_{\tau}=\int_{\tau}^{\infty} e^{-r(t-\tau)}\left(\frac{(1-\alpha)\left(k_{t}-k_{\tau}\right)}{k_{t}\left(N-k_{\tau}\right)}\right)\right) A k_{t}^{1-\beta} d t .
$$

Inserting Eq. (51) into Eq. (54), we derive

$$
u_{\tau}=\int_{\tau}^{\infty} e^{-r(t-\tau)}\left(\frac{(1-\alpha)\left(e^{\gamma N(t-\tau)}-1\right)}{N e^{\gamma N(t-\tau)}}\right) A k_{t}^{1-\beta} d t
$$


Again, defining $s=t-\tau$, Eq. (55) becomes

$$
u_{\tau}=\int_{0}^{\infty} e^{-r s}\left(\frac{(1-\alpha)\left(e^{\gamma N s}-1\right)}{N e^{\gamma N s}}\right) A k_{\tau+s}^{1-\beta} d s .
$$

Equation (56) yields that if $\beta=1, u_{\tau}$ is a constant that does not vary with $\tau$; if $\beta>1, k_{\tau+s}^{1-\beta}$ decreases with $k_{\tau}$ so $u_{\tau}$ decreases with $\tau$; and if $\beta<1, k_{\tau+s}^{1-\beta}$ increases with $k_{\tau}$ so $u_{\tau}$ increases with $\tau$. Moreover, combining Eqs. (53) and (56), we have

$$
v_{\tau}^{\tau}-u_{\tau}=\int_{0}^{\infty} e^{-r s}\left(1-\alpha+\alpha \frac{k_{\tau+s}}{k_{\tau}}-\frac{(1-\alpha)\left(e^{\gamma N s}-1\right)}{N e^{\gamma N s}} k_{\tau+s}\right) A k_{\tau+s}^{-\beta} d s .
$$

Within the integral of Eq. (57), both terms $\left(1-\alpha+\alpha \frac{k_{\tau+s}}{k_{\tau}}-\frac{(1-\alpha)\left(e^{\gamma N s}-1\right)}{N e^{\gamma N s}} k_{\tau+s}\right)$ and $k_{\tau+s}^{-\beta}$ decrease in $\tau$, so $v_{\tau}^{\tau}-u_{\tau}$ decreases with $\tau$. Therefore, given the free entry condition $v_{0}^{0}-u_{0}=c$, we have $v_{\tau}^{\tau}-u_{\tau}<c$ for any $\tau>0$, so no innovator would enter the industry after date 0 .

(B) Note that $v_{0}=v_{0}^{0}$. Equations (49) and (54) yield that

$$
v_{0}-u_{0}=\frac{1}{N-k_{0}} \int_{0}^{\infty} e^{-r t}\left(\alpha \frac{N}{k_{0}}+(1-\alpha) \frac{N}{k_{t}}-1\right) A k_{t}^{1-\beta} d t
$$

The free entry condition $v_{0}-u_{0}=c$ then pins down the entry of innovators $k_{0}$ at date 0, as shown by Eq. (13).

Full dynamic paths. - The proof above confirms that innovators only enter at date 0 , so the time path of firm numbers is given by Eq. (4). Following that, the full dynamic paths of $\omega_{t}, v_{t}$ and $v_{t}^{\tau}$ for any $t \geq 0$ can be derived. Besides, the time path of $u_{t}$ has been solved above (cf. Eq. (56)). Recall Eq. (7) that

$$
r \omega_{t}=p_{t}+\frac{d \omega_{t}}{d t}
$$

which yields that

$$
\omega_{t}=\int_{t}^{\infty} e^{-r(s-t)} p_{s} d s=\int_{t}^{\infty} e^{-r(s-t)} A k_{s}^{-\beta} d s
$$

Because $k_{s}$ increases in $s, \omega_{t}$ declines in $t$.

Consider a marginal innovator who enters at date $\tau \geq 0$. From any date $t \geq \tau$, he collects $A k_{s}^{-\beta}$ in each period $s \geq t$ by selling goods, and collect a fraction $\frac{\alpha\left(k_{s}-k_{t}\right)}{k_{s} k_{\tau}}$ of the total industry revenues $A k_{s}^{1-\beta}$ from new entrants after date $t$ by selling ideas. Therefore, his value at date $t$ is determined by

$$
v_{t}^{\tau}=A \int_{t}^{\infty} e^{-r(s-t)} k_{s}^{-\beta} d s+\frac{A}{k_{\tau}} \int_{t}^{\infty} e^{-r(s-t)} \alpha\left(1-\frac{k_{t}}{k_{s}}\right) k_{s}^{1-\beta} d s .
$$


Because $k_{\tau}$ increases in $\tau, v_{t}^{\tau}$ declines in $\tau$.

Finally, Eq. (60) suggests that an innovator who entered at date 0 has value $v_{t}$ for any $t \geq 0$ :

$$
v_{t}=v_{t}^{0}=A \int_{t}^{\infty} e^{-r(s-t)} k_{s}^{-\beta} d s+\frac{A}{k_{0}} \int_{t}^{\infty} e^{-r(s-t)} \alpha\left(1-\frac{k_{t}}{k_{s}}\right) k_{s}^{1-\beta} d s .
$$

Equation (61) suggests that the time path of $v_{t}$ depends on parameter values. For example, it is easy to see $v_{t}$ decreases in $t$ when $\alpha=0$ or when $\beta \geq 1$, and one can also prove that $v_{t}$ may initially increase and later decrease in $t$ if $\alpha$ is close to $1, \beta$ is close to zero, and $c$ is large enough (A formal proof is available upon request).

A special-case example. - The differential equations (7)-(9) can be solved directly when demand is unit elastic (i.e., $\beta=1$ ). We show the result is consistent with our general solution above. Again, we start with the conjecture that at equilibrium no agent would enter as an innovator after date 0 , so the number of firms $k_{t}$ evolve as described by Eq. (4). To simplify the notation, we define $b=\frac{N}{k_{0}}-1$.

An imitator's value $\omega_{t}$ satisfies the ordinary differential equation (ODE) introduced by Eq. (7):

$$
r \omega_{t}=p_{t}+\frac{d \omega_{t}}{d t}=A\left(\frac{N e^{N \gamma t}}{e^{N \gamma t}+b}\right)^{-1}+\frac{d \omega_{t}}{d t}
$$

The ODE has the unique bounded solution satisfying $\omega_{t \rightarrow \infty}<\infty$ that

$$
\omega_{t}=\frac{A}{N r}+\frac{A b e^{-N \gamma t}}{N(r+N \gamma)} \text {. }
$$

An innovator's value $v_{t}$ satisfies the ODE introduced by Eq. (8):

$$
\begin{aligned}
r v_{t} & =p_{t}+\frac{d k_{t} / d t}{k_{0}} \alpha \omega_{t}+\frac{d v_{t}}{d t} \\
& =A\left(\frac{N e^{N \gamma t}}{e^{N \gamma t}+b}\right)^{-1}+\frac{\gamma(1+b) \alpha A b e^{N \gamma t}}{\left(e^{N \gamma t}+b\right)^{2}}\left(\frac{1}{r}+\frac{b}{e^{N \gamma t}(r+N \gamma)}\right)+\frac{d v_{t}}{d t}
\end{aligned}
$$

Solving the ODE and imposing $v_{t}$ is bounded as $t \rightarrow \infty$ yields

$$
v_{t}=\frac{A}{N r}+\frac{A\left((r+b \alpha N \gamma+\alpha N \gamma) b e^{N \gamma t}+b^{2} r\right)}{N r(r+N \gamma)\left(e^{2 N \gamma t}+b e^{N \gamma t}\right)}
$$

Equation (63) implies that at date 0, we have

$$
v_{0}=\frac{A}{N r}+\frac{A b(\alpha N \gamma+r)}{N r(r+N \gamma)}
$$

and the value of being an innovator declines over time (i.e., $d v_{t} / d t<0$ ). 
The option value of being an outsider $u_{t}$ satisfies the ODE introduced by Eq. (9):

$$
\begin{aligned}
r u_{t} & =\gamma k_{t}\left[(1-\alpha) \omega_{t}-u_{t}\right]+\frac{d u_{t}}{d t} \\
& =\frac{\gamma N e^{N \gamma t}}{e^{N \gamma t}+b}\left[(1-\alpha)\left(\frac{A}{N r}+\frac{A b e^{-N \gamma t}}{N(r+N \gamma)}\right)-u_{t}\right]+\frac{d u_{t}}{d t} .
\end{aligned}
$$

The unique bounded solution for $u_{t}$ is a constant:

$$
u_{t}=u=\frac{A \gamma(1-\alpha)}{r^{2}+N \gamma r}
$$

Note that if an agent deviates from the equilibrium and enters at date $t>0$, he would have a lower value than an innovator who entered at date 0 (i.e., $v_{t}^{t}<v_{t}^{0}$ ) because the latter would have a larger family of imitators to disseminate his idea and collect idea-sale revenues. Therefore, the finding that $v_{t}-u_{t}$ declines in $t$ implies that $v_{t}^{t}-u_{t}<c$ at any date $t>0$, so no agent would enter as an innovator after date 0 .

At $t=0$, given the free entry condition requires $v_{0}-u_{0}=c$, Eqs. (64) and (65) yield

$$
b=\frac{c N r(r+N \gamma)-A N \gamma \alpha-A r}{A(\alpha N \gamma+r)} .
$$

Since $b=\frac{N}{k_{0}}-1$, we derive the equilibrium entry of innovators at date 0 , denoted by $k_{0}^{\mathbf{I}}$ for Regime 1 , to be

$$
k_{0}^{\mathbf{I}}=\frac{A(\alpha N \gamma+r)}{c r(r+N \gamma)},
$$

which is consistent with the general solution above, shown by Eq. (13) for $\beta=1$.

\section{A.2. Proof of Proposition 2}

Proof. (A) In Regime 2, all firms at date- $t$ share the same value $v_{t}$ regardless of their entry date or type. In this case, we can characterize the differential equations (10)-(11) directly. We first conjecture that no agent would enter as an innovator after date 0 , so the time path of firm numbers is determined by Eq. (4) that

$$
k_{t}=\frac{N e^{\gamma N t}}{e^{\gamma N t}+\frac{N}{k_{0}}-1} .
$$

Then, $v_{t}$ is determined by Eq. (10) that

$$
\begin{aligned}
r v_{t} & =p_{t}+\gamma\left(N-k_{t}\right) \alpha v_{t}+\frac{d v_{t}}{d t} \\
\frac{d v_{t}}{d t}-\left[r-\gamma\left(N-k_{t}\right) \alpha\right] v_{t} & =-p_{t} .
\end{aligned}
$$


Defining $z_{t}=\exp \left(\int-\left[r-\gamma\left(N-k_{t}\right) \alpha\right] d t\right)$, we can rewrite Eq. (67) as

$$
\frac{d\left(z_{t} v_{t}\right)}{d t}=-z_{t} p_{t}
$$

which yields the general solution

$$
v_{t}=z_{t}^{-1} \int-z_{t} p_{t} d t+z_{t}^{-1} C
$$

where $C$ is a constant of integration.

Given that $k_{t}=\frac{N e^{\gamma N t}}{e^{\gamma N t}+\frac{N}{k_{0}}-1}$, we can solve $z_{t}$ :

$$
z_{t}=\exp \left(\int-\left[r-\gamma\left(N-k_{t}\right) \alpha\right] d t\right)=e^{-r t}\left(\frac{N-k_{0}}{k_{0}} e^{-\gamma N t}+1\right)^{-\alpha} .
$$

Accordingly,

$$
\begin{aligned}
v_{t}= & e^{r t}\left(\frac{N-k_{0}}{k_{0}} e^{-\gamma N t}+1\right)^{\alpha} \int_{\alpha}-e^{-r t}\left(\frac{N-k_{0}}{k_{0}} e^{-\gamma N t}+1\right)^{-\alpha} p_{t} d t \\
& +e^{r t}\left(\frac{N-k_{0}}{k_{0}} e^{-\gamma N t}+1\right)^{\alpha} C,
\end{aligned}
$$

which requires $C=0$ given that $v_{t}$ needs to be bounded as $t \rightarrow \infty$. We then solve $v_{t}$ as follows:

$$
\begin{aligned}
v_{t} & =e^{r t}\left(\frac{N-k_{0}}{k_{0}} e^{-\gamma N t}+1\right)^{\alpha} \int-e^{-r t}\left(\frac{N-k_{0}}{k_{0}} e^{-\gamma N t}+1\right)^{-\alpha} p_{t} d t \\
& =e^{r t}\left(\frac{N-k_{0}}{k_{0}} e^{-\gamma N t}+1\right)^{\alpha} \int_{t}^{\infty} e^{-r s}\left(\frac{N-k_{0}}{k_{0}} e^{-\gamma N s}+1\right)^{-\alpha} p_{s} d s \\
& =\int_{t}^{\infty} e^{-r(s-t)}\left(\frac{\frac{N-k_{0}}{k_{0}} e^{-\gamma N s}+1}{\frac{N-k_{0}}{k_{0}} e^{-\gamma N t}+1}\right)^{-\alpha} p_{s} d s \\
& =\int_{t}^{\infty} e^{-r(s-t)}\left(1-\frac{1-e^{-\gamma N(s-t)}}{\frac{k_{0}}{N-k_{0}} e^{\gamma N t}+1}\right)^{-\alpha} p_{s} d s .
\end{aligned}
$$

Defining $i=s-t$, we can rewrite Eq.(68) as

$$
v_{t}=\int_{0}^{\infty} e^{-r i}\left(1-\frac{1-e^{-\gamma N i}}{\frac{k_{0}}{N-k_{0}} e^{\gamma N t}+1}\right)^{-\alpha} p_{t+i} d i
$$

In the integral, both terms $\left(1-\frac{1-e^{-\gamma N i}}{\frac{k_{0}}{N-k_{0}} e^{\gamma N t}+1}\right)^{-\alpha}$ and $p_{t+i}=A k_{t+i}^{-\beta}$ decrease in $t$. Therefore, $v_{t}$ decreases with $t$. 
Next, we show $v_{t}-u_{t}$ decreases with $t$. Recall that in Regime $2, u_{t}$ is determined by Eq. (11) that

$$
r u_{t}=\gamma k_{t}\left((1-\alpha) v_{t}-u_{t}\right)+\frac{d u_{t}}{d t}
$$

which, together with Eq. (10), implies that

$$
\frac{d\left(v_{t}-u_{t}\right)}{d t}=\left(r+\gamma k_{t}\right)\left(v_{t}-u_{t}\right)-\left(p_{t}+\gamma N \alpha v_{t}\right)
$$

Defining $\psi_{t} \equiv v_{t}-u_{t}$, we can rewrite Eq. (69) as

$$
\frac{d \psi_{t}}{d t}-\left(r+\gamma k_{t}\right) \psi_{t}=-\left(p_{t}+\gamma N \alpha v_{t}\right)
$$

Define $z_{t}=\exp \int-\left(r+\gamma k_{t}\right) d t$. We then have

$$
\frac{d\left(z_{t} \psi_{t}\right)}{d t}=-z_{t}\left(p_{t}+\gamma N \alpha v_{t}\right)
$$

which yields the general solution

$$
\psi_{t}=z_{t}^{-1} \int-z_{t}\left(p_{t}+\gamma N \alpha v_{t}\right) d t+z_{t}^{-1} C
$$

where $C$ is a constant of integration.

Given that $k_{t}=\frac{N e^{\gamma N t}}{e^{\gamma N t}+\frac{N}{k_{0}}-1}$, we can solve $z_{t}$ :

$$
z_{t}=e^{-r t} \frac{k_{0}}{N-k_{0}+k_{0} e^{\gamma N t}}
$$

Again, $\psi_{t}$ needs to be bounded as $t \rightarrow \infty$, so $C=0$. We then have

$$
\begin{aligned}
\psi_{t} & =z_{t}^{-1} \int-z_{t}\left(p_{t}+\gamma N \alpha v_{t}\right) d t \\
& =e^{r t} \frac{N-k_{0}+k_{0} e^{\gamma N t}}{k_{0}} \int-e^{-r t} \frac{k_{0}}{N-k_{0}+k_{0} e^{\gamma N t}}\left(p_{t}+\gamma N \alpha v_{t}\right) d t \\
& =e^{r t} \frac{N-k_{0}+k_{0} e^{\gamma N t}}{k_{0}} \int_{t}^{\infty} e^{-r s} \frac{k_{0}}{N-k_{0}+k_{0} e^{\gamma N s}}\left(p_{s}+\gamma N \alpha v_{s}\right) d s \\
& =\int_{t}^{\infty} e^{-r(s-t)}\left(1-\frac{e^{\gamma N(s-t)}-1}{\left(\frac{N-k_{0}}{k_{0}}\right) e^{-\gamma N t}+e^{\gamma N(s-t)}}\right)\left(p_{s}+\gamma N \alpha v_{s}\right) d s .
\end{aligned}
$$

Define $i=s-t$. We can rewrite Eq. (70) as

$$
\psi_{t}=\int_{0}^{\infty} e^{-r i}\left(1-\frac{e^{\gamma N i}-1}{\left(\frac{N-k_{0}}{k_{0}}\right) e^{-\gamma N t}+e^{\gamma N i}}\right)\left(p_{t+i}+\gamma N \alpha v_{t+i}\right) d i .
$$


Note that in the integral, both terms $\left(1-\frac{e^{\gamma N i}-1}{\left(\frac{N-k_{0}}{k_{0}}\right) e^{-\gamma N t}+e^{\gamma N i}}\right)$ and $\left(p_{t+i}+\gamma N \alpha v_{t+i}\right)$ decrease in $t$, hence $\psi_{t}=v_{t}-u_{t}$ strictly decreases with $t$. Given the free entry condition that $v_{0}-u_{0}=c$ at date 0 , we know $v_{t}-u_{t}<c$ at any date $t>0$, so no agent would enter as an innovator after date 0 .

(B) Equation (68) implies that

$$
v_{t}=\int_{t}^{\infty} e^{-r(s-t)}\left(\frac{k_{s}}{k_{t}}\right)^{\alpha} p_{s} d s
$$

so that

$$
v_{0}=\int_{0}^{\infty} e^{-r t}\left(\frac{k_{t}}{k_{0}}\right)^{\alpha} A k_{t}^{-\beta} d t
$$

At date 0 , the total industry discounted revenue, $\int_{0}^{\infty} e^{-r t} A k_{t}^{1-\beta} d t$, is shared by the two groups - the initial incumbents $k_{0}$ and the outsiders $N-k_{0}$. With the free entry condition $v_{0}-c=u_{0}$ we have

$$
\int_{0}^{\infty} e^{-r t} A k_{t}^{1-\beta} d t=v_{0} k_{0}+u_{0}\left(N-k_{0}\right)=v_{0} N-c\left(N-k_{0}\right) .
$$

Plugging Eq. (71) into Eq. (72) yields Eq. (14).

\section{A.3. Proof of Proposition 3}

Proof. In Regime 1, $k_{0}^{\mathbf{I}}$ is determined by Eq. (13) that

$$
\frac{1}{N-k_{0}} \int_{0}^{\infty} e^{-r t}\left(\alpha \frac{N}{k_{0}}+(1-\alpha) \frac{N}{k_{t}}-1\right) A k_{t}^{1-\beta} d t=c .
$$

Note that as $k_{0} \rightarrow N$, we have $k_{t} \rightarrow N$. So both the numerator and the denominator of the left hand side of Eq. (13) goes to 0 as $k_{0} \rightarrow N$. Applying L'Hôpital's rule, the left hand side converges to

$$
\begin{aligned}
& \lim _{k_{0} \rightarrow N} \frac{\int_{0}^{\infty} e^{-r t}\left(\alpha \frac{N}{k_{0}}+(1-\alpha) \frac{N}{k_{t}}-1\right) A k_{t}^{1-\beta} d t}{N-k_{0}} \\
= & \frac{\lim _{k_{0} \rightarrow N} \int_{0}^{\infty} e^{-r t}\left\{\begin{array}{c}
\left(-\alpha k_{0}^{-2} N-(1-\alpha) N k_{t}^{-2} \frac{\partial k_{t}}{\partial k_{0}}\right) A k_{t}^{1-\beta} \\
+\left(\alpha \frac{N}{k_{0}}+(1-\alpha) \frac{N}{k_{t}}-1\right)(1-\beta) A k_{t}^{-\beta} \frac{\partial k_{t}}{\partial k_{0}}
\end{array}\right\} d t}{-1} \\
= & \lim _{k_{0} \rightarrow N} \int_{0}^{\infty} e^{-r t}\left(\alpha+(1-\alpha) e^{-\gamma N t}\right) A N^{-\beta} d t=\frac{\alpha \gamma N+r}{r(r+\gamma N)} A N^{-\beta} .
\end{aligned}
$$

Proposition 4 shows that $d k_{0}^{\mathbf{I}} / d c<0$. Therefore, the model has an interior solution $k_{0}^{\mathbf{I}}<N$ in Regime 1 iff $c>\frac{(\alpha \gamma N+r)}{r(r+\gamma N)} A N^{-\beta}$. 
Similarly, $k_{0}^{\mathbf{I I}}$ is determined by Eq. (14) that

$$
\frac{1}{N-k_{0}} \int_{0}^{\infty} e^{-r t}\left(\left(\frac{N}{k_{0}}\right)^{\alpha}\left(\frac{N}{k_{t}}\right)^{1-\alpha}-1\right) A k_{t}^{1-\beta} d t=c .
$$

As $k_{0} \rightarrow N$, applying L'Hôpital's rule, the left hand side of Eq. (14) converges to $\frac{\alpha \gamma N+r}{r(r+\gamma N)} A N^{-\beta}$. Proposition 4 shows that $d k_{0}^{\mathbf{I I}} / d c<0$. Therefore, the model has an interior solution $k_{0}^{\mathbf{I I}}<N$ in Regime 2 iff $c>\frac{(\alpha \gamma N+r)}{r(r+\gamma N)} A N^{-\beta}$.

\section{A.4. Proof of Proposition 4}

Proof. (A) We first prove that $k_{0}^{\mathbf{I}}$ increases with $\alpha$ and $A$, but decreases with $c$ and $r$. Rewrite Eq. (13) as

$$
G=\int_{0}^{\infty} e^{-r t} F\left(t ; k_{0}\right) d t-c=0
$$

where

$$
F\left(t ; k_{0}\right)=\frac{1}{N-k_{0}}\left(\alpha \frac{N}{k_{0}}+(1-\alpha) \frac{N}{k_{t}}-1\right) A k_{t}^{1-\beta}
$$

and

$$
k_{t}=\frac{N e^{\gamma N t}}{e^{\gamma N t}+\frac{N}{k_{0}}-1} .
$$

We verify that $\frac{\partial F\left(t ; k_{0}\right)}{\partial k_{0}}<0$, so that $\frac{\partial G}{\partial k_{0}}<0$. Therefore,

$$
\begin{gathered}
\frac{\partial k_{0}^{\mathbf{I}}}{\partial \alpha}=-\frac{\partial G / \partial \alpha}{\partial G / \partial k_{0}^{\mathbf{I}}}>0 ; \quad \frac{\partial k_{0}^{\mathbf{I}}}{\partial A}=-\frac{\partial G / \partial A}{\partial G / \partial k_{0}^{\mathbf{I}}}>0 ; \\
\frac{\partial k_{0}^{\mathbf{I}}}{\partial c}=-\frac{\partial G / \partial c}{\partial G / \partial k_{0}^{\mathbf{I}}}<0 ; \frac{\partial k_{0}^{\mathbf{I}}}{\partial r}=-\frac{\partial G / \partial r}{\partial G / \partial k_{0}^{\mathbf{I}}}<0 .
\end{gathered}
$$

Similarly, with Eq. (14), we can prove that $k_{0}^{\mathbf{I I}}$ increases with $\alpha$ and $A$, but decreases with $c$ and $r$.

(B) First, it is straightforward to verify Eqs. (13) and (14) are identical when $\alpha \in\{0,1\}$, so $k_{0}^{\mathbf{I}}=k_{0}^{\mathbf{I I}}$.

Second, for any $\alpha \in(0,1)$ and $t>0$, we can apply the mean-value theorem and derive

$$
\left(\frac{N}{k_{0}}\right)^{\alpha}\left(\frac{N}{k_{t}}\right)^{1-\alpha}=\left(\frac{N}{k_{t}}\right)\left(\frac{k_{t}}{k_{0}}\right)^{\alpha}=\left(\frac{N}{k_{t}}\right)\left(1+\alpha\left(\frac{k^{\prime}}{k_{0}}\right)^{\alpha-1} \frac{\left(k_{t}-k_{0}\right)}{k_{0}}\right)
$$

where $k_{t}>k^{\prime}>k_{0}$. Therefore,

$$
\left(\frac{N}{k_{0}}\right)^{\alpha}\left(\frac{N}{k_{t}}\right)^{1-\alpha}<\left(\frac{N}{k_{t}}\right)\left(1+\alpha \frac{\left(k_{t}-k_{0}\right)}{k_{0}}\right)=\alpha \frac{N}{k_{0}}+(1-\alpha) \frac{N}{k_{t}} .
$$


Given that $k_{0}^{\mathbf{I}}$ and $k_{t}^{\mathbf{I}}$ satisfy Eq. (13) that

$$
\int_{0}^{\infty} e^{-r t} \frac{1}{N-k_{0}^{\mathbf{I}}}\left(\alpha \frac{N}{k_{0}^{\mathbf{I}}}+(1-\alpha) \frac{N}{k_{t}^{\mathbf{I}}}-1\right) A\left(k_{t}^{\mathbf{I}}\right)^{1-\beta} d t=c,
$$

the same $k_{0}^{\mathbf{I}}$ and $k_{t}^{\mathbf{I}}$ would not satisfy Eq. (14). Instead,

$$
\int_{0}^{\infty} e^{-r t} \frac{1}{N-k_{0}^{\mathbf{I}}}\left(\left(\frac{N}{k_{0}^{\mathbf{I}}}\right)^{\alpha}\left(\frac{N}{k_{t}^{\mathbf{I}}}\right)^{1-\alpha}-1\right) A\left(k_{t}^{\mathbf{I}}\right)^{1-\beta} d t<c,
$$

given the inequality (73).

The left-hand side of Eq. (14) can be written as

$$
L H S=\int_{0}^{\infty} e^{-r t} F\left(t ; k_{0}\right) d t
$$

where

$$
F\left(t ; k_{0}\right)=\frac{1}{N-k_{0}}\left(\left(\frac{N}{k_{0}}\right)^{\alpha}\left(\frac{N}{k_{t}}\right)^{1-\alpha}-1\right) A k_{t}^{1-\beta}
$$

and

$$
k_{t}=\frac{N e^{\gamma N t}}{e^{\gamma N t}+\frac{N}{k_{0}}-1} .
$$

We verify that $\frac{\partial F\left(t ; k_{0}\right)}{\partial k_{0}}<0$, so that $\frac{\partial L H S}{\partial k_{0}}<0$. Therefore, the solution $k_{0}^{\text {II }}$ that satisfies (14) has to satisfy $k_{0}^{\text {II }}<k_{0}^{\mathbf{I}}$.

\section{A.5. Proof of Proposition 5}

Proof. (i) We first prove the results for Regime 1. Rewrite Eq. (13) as

$$
G=\int_{0}^{\infty} e^{-r t} F(t ; \gamma) d t-\frac{c}{A N^{1-\beta}}=0,
$$

where

$$
F(t ; \gamma) d t=\frac{1}{\left(N-k_{0}\right)}\left(\alpha \frac{N}{k_{0}}-1+(1-\alpha)\left(1+\left(\frac{N}{k_{0}}-1\right) e^{-\gamma N t}\right)\right)\left(1+\left(\frac{N}{k_{0}}-1\right) e^{-\gamma N t}\right)^{\beta-1} .
$$

Note that

$$
\frac{F(t ; \gamma)}{\partial \gamma} \propto\left\{\begin{array}{c}
-(1-\alpha)\left(1+\left(\frac{N}{k_{0}}-1\right) e^{-\gamma N t}\right) \\
-\left(\alpha \frac{N}{k_{0}}-1+(1-\alpha)\left(1+\left(\frac{N}{k_{0}}-1\right) e^{-\gamma N t}\right)\right)(\beta-1)
\end{array}\right\} .
$$


Therefore, for $\beta \geq 1$, we have $\partial G / \partial \gamma<0$ (except $\partial G / \partial \gamma=0$ when $\beta=\alpha=1$ ). Recall that $\partial G / \partial k_{0}<0$ from the proof of Proposition 4 , so we derive

$$
\frac{\partial k_{0}^{\mathbf{I}}}{\partial \gamma}=-\frac{\partial G / \partial \gamma}{\partial G / \partial k_{0}^{\mathbf{I}}}<0\left(\text { except } \frac{\partial k_{0}^{\mathbf{I}}}{\partial \gamma}=0 \text { when } \beta=\alpha=1\right) \text {. }
$$

In contrast, for $\beta<1$,

$$
\frac{F(t ; \gamma)}{\partial \gamma}<0 \Longleftrightarrow(1-\alpha) \beta\left(1+\left(\frac{N}{k_{0}}-1\right) e^{-\gamma N t}\right)>\left(\alpha \frac{N}{k_{0}}-1\right)(1-\beta),
$$

which holds for any $t \geq 0$ if

$$
(1-\alpha) \beta>\left(\alpha \frac{N}{k_{0}}-1\right)(1-\beta) \Longleftrightarrow \alpha<\frac{1}{\frac{N}{k_{0}}(1-\beta)+\beta} .
$$

Therefore, we have

$$
\frac{\partial k_{0}^{\mathbf{I}}}{\partial \gamma}=-\frac{\partial G / \partial \gamma}{\partial G / \partial k_{0}^{\mathbf{I}}}<0 \text { if } \alpha<\frac{1}{\frac{N}{k_{0}^{\mathbf{I}}}(1-\beta)+\beta}
$$

Similarly, for $\beta<1$,

$$
\frac{F(t ; \gamma)}{\partial \gamma}>0 \Longleftrightarrow(1-\alpha)\left(1+\left(\frac{N}{k_{0}}-1\right) e^{-\gamma N t}\right) \beta<\left(\alpha \frac{N}{k_{0}}-1\right)(1-\beta),
$$

which holds for any $t \geq 0$ if

$$
(1-\alpha)\left(\frac{N}{k_{0}}\right) \beta<\left(\alpha \frac{N}{k_{0}}-1\right)(1-\beta) \Longleftrightarrow \alpha>\beta+\frac{k_{0}}{N}(1-\beta) .
$$

Therefore, we have

$$
\frac{\partial k_{0}^{\mathbf{I}}}{\partial \gamma}=-\frac{\partial G / \partial \gamma}{\partial G / \partial k_{0}^{\mathbf{I}}}>0 \text { if } \alpha>\beta+\frac{k_{0}^{\mathbf{I}}}{N}(1-\beta) .
$$

(ii) We now prove the results for Regime 2. Rewrite Eq. (14) as

$$
G=\int_{0}^{\infty} e^{-r t} F(t ; \gamma) d t-\frac{c}{A N^{1-\beta}}=0
$$

where

$$
F(t ; \gamma) d t=\left(\left(\frac{N}{k_{0}}\right)^{\alpha}\left(1+\left(\frac{N}{k_{0}}-1\right) e^{-\gamma N t}\right)^{1-\alpha}-1\right)\left(1+\left(\frac{N}{k_{0}}-1\right) e^{-\gamma N t}\right)^{\beta-1} .
$$


Note that

$$
\begin{aligned}
& \frac{\partial F(t ; \gamma)}{\partial \gamma} \propto \\
& \left\{\begin{array}{c}
-\left(\frac{N}{k_{0}}\right)^{\alpha}(1-\alpha)\left(1+\left(\frac{N}{k_{0}}-1\right) e^{-\gamma N t}\right)^{1-\alpha} \\
+\left(\left(\frac{N}{k_{0}}\right)^{\alpha}\left(1+\left(\frac{N}{k_{0}}-1\right) e^{-\gamma N t}\right)^{1-\alpha}-1\right)(1-\beta)
\end{array}\right\} .
\end{aligned}
$$

Therefore, for $\beta \geq 1$, we have $\partial G / \partial \gamma<0$ (except $\partial G / \partial \gamma=0$ when $\beta=\alpha=1$ ). Recall that $\partial G / \partial k_{0}<0$ from the proof of Proposition 4 so we derive

$$
\frac{\partial k_{0}^{\mathbf{I I}}}{\partial \gamma}=-\frac{\partial G / \partial \gamma}{\partial G / \partial k_{0}^{\mathbf{I I}}}<0\left(\text { except } \frac{\partial k_{0}^{\mathbf{I I}}}{\partial \gamma}=0 \text { when } \beta=\alpha=1\right) .
$$

In contrast, for $\beta<1$,

$$
\frac{\partial F(t ; \gamma)}{\partial \gamma}<0 \Longleftrightarrow\left(\frac{N}{k_{0}}\right)^{\alpha}\left(1+\left(\frac{N}{k_{0}}-1\right) e^{-\gamma N t}\right)^{1-\alpha}(\alpha-\beta)<(1-\beta),
$$

which holds for any $t \geq 0$ if

$$
\left(\frac{N}{k_{0}}\right)(\alpha-\beta)<(1-\beta) \Longleftrightarrow \alpha<\frac{k_{0}}{N}(1-\beta)+\beta .
$$

Therefore, we have

$$
\frac{\partial k_{0}^{\mathbf{I I}}}{\partial \gamma}=-\frac{\partial G / \partial \gamma}{\partial G / \partial k_{0}^{\mathbf{I I}}}<0 \text { if } \alpha<\frac{k_{0}^{\mathbf{I I}}}{N}(1-\beta)+\beta .
$$

Similarly, for $\beta<1$,

$$
\frac{\partial F(t ; \gamma)}{\partial \gamma}>0 \Longleftrightarrow\left(\frac{N}{k_{0}}\right)^{\alpha}\left(1+\left(\frac{N}{k_{0}}-1\right) e^{-\gamma N t}\right)^{1-\alpha}(\alpha-\beta)>(1-\beta),
$$

which holds for any $t \geq 0$ if

$$
\left(\frac{N}{k_{0}}\right)^{\alpha}(\alpha-\beta)>(1-\beta) \Longleftrightarrow \alpha>\left(\frac{k_{0}}{N}\right)^{\alpha}(1-\beta)+\beta .
$$

Therefore, we have

$$
\frac{\partial k_{0}^{\mathbf{I I}}}{\partial \gamma}=-\frac{\partial G / \partial \gamma}{\partial G / \partial k_{0}^{\mathbf{I I}}}>0 \text { if } \alpha>\left(\frac{k_{0}^{\mathbf{I I}}}{N}\right)^{\alpha}(1-\beta)+\beta .
$$




\section{A.6. Proof of Proposition 6}

Proof. (A) Consider the case $\beta<1$ first. For any date $\tau \geq 0$, if no further innovators enter, the number of firms at dates $t \geq \tau$ is

$$
k_{t}=\frac{N e^{\gamma N(t-\tau)}}{e^{\gamma N(t-\tau)}+\frac{N}{k_{\tau}}-1} \quad \text { for } t \geq \tau .
$$

As of date $\tau$, the social return to innovation is

$$
S R_{\tau}=\int_{\tau}^{\infty} e^{-r(t-\tau)} \frac{A}{1-\beta} k_{t}^{1-\beta} d t .
$$

The current cost of innovation is $c$ per unit, and its marginal social return (even if no further innovations are made) is

$$
\frac{\partial S R_{\tau}}{\partial k_{\tau}}=\int_{\tau}^{\infty} e^{-r(t-\tau)} A k_{t}^{-\beta} \frac{\partial k_{t}}{\partial k_{\tau}} d t,
$$

where

$$
\frac{\partial k_{t}}{\partial k_{\tau}}=\frac{N^{2} e^{\gamma N(t-\tau)}}{\left(N+\left(e^{\gamma N(t-\tau)}-1\right) k_{\tau}\right)^{2}},
$$

which is strictly decreasing in $k_{\tau}$. And since $k_{t}$ is increasing in $t$,

$$
e^{-r(t-\tau)} k_{t}^{-\beta} \frac{\partial k_{t}}{\partial k_{\tau}}
$$

is also decreasing in $k_{\tau}$. Therefore $\frac{\partial S R_{\tau}}{\partial k_{\tau}}$ is also strictly decreasing in $k_{\tau}$ and so if at date zero $k_{0}$ is chosen so that $\frac{\partial S R_{0}}{\partial k_{0}}=c$, thereafter $\frac{\partial S R_{\tau}}{\partial k_{\tau}}<c$. Similarly, we can prove the result holds for $\beta \geq 1$. Hence, it is socially optimal to innovate only at date zero.

(B) Since it is socially optimal to innovate only at date zero, the planner should choose $k_{0}^{*}$ to maximize social welfare:

$$
\max _{k_{0}}\left\{\int_{0}^{\infty} e^{-r t} U\left(k_{t}\right) d t-c k_{0}\right\},
$$

subject to Eq. (4). We can verify that the objective function is strictly concave in $k_{0}$, so the socially optimal number of innovators $k_{0}^{*}$ is pinned down by the first-order condition which is Eq. (24) for any $\beta>0$.

(C) Given that the social welfare function (78) is strictly concave in $k_{0}$, for $k_{0}^{*}<N$ to hold, one needs

$$
\left.\frac{d\left\{\int_{0}^{\infty} e^{-r t} U\left(k_{t}\right) d t-c k_{0}\right\}}{d k_{0}}\right|_{k_{0}=N}<0,
$$

which yields condition (25). 


\section{A.7. Proof of Proposition 7}

Proof. (A) Rewriting Eq. (24), we define

$$
G=\int_{0}^{\infty} e^{-(r+\gamma N) t} \frac{A}{k_{0}^{* 2}}\left(\frac{N e^{\gamma N t}}{e^{\gamma N t}+\left(\frac{N}{k_{0}^{*}}-1\right)}\right)^{2-\beta} d t-c=0 .
$$

It follows that

$$
\frac{\partial k_{0}^{*}}{\partial A}=-\frac{\partial G / \partial A}{\partial G / \partial k_{0}^{*}}>0 .
$$

Similarly, we can prove $\partial k_{0}^{*} / \partial c<0$ and $\partial k_{0}^{*} / \partial r<0$.

The sign of $\partial k_{0}^{*} / \partial \gamma$ depends on $\partial G / \partial \gamma$ and requires some discussions.

$\frac{\partial G}{\partial \gamma} \propto \int_{0}^{\infty} e^{-r t}\left\{\begin{array}{c}(1-\beta)\left(1+\left(\frac{N}{k_{0}^{*}}-1\right) e^{-\gamma N t}\right)^{\beta-2}\left(e^{\gamma N t}+\left(\frac{N}{k_{0}^{*}}-1\right)\right)^{-1}\left(\frac{N}{k_{0}^{*}}-1\right) e^{-\gamma N t} N t \\ -\left(1+\left(\frac{N}{k_{0}^{*}}-1\right) e^{-\gamma N t}\right)^{\beta-1}\left(e^{\gamma N t}+\left(\frac{N}{k_{0}^{*}}-1\right)\right)^{-2} e^{\gamma N t} N t\end{array}\right\} d t$

This implies $\frac{\partial G}{\partial \gamma}<0$ if $\beta \geq 1$. When $\beta<1$, the sign of $\frac{\partial G}{\partial \gamma}<0$ if $(1-\beta)\left(\frac{N}{k_{0}^{*}}-1\right)<e^{\gamma N t}$. A sufficient condition is that

$$
(1-\beta)\left(\frac{N}{k_{0}^{*}}-1\right)<1
$$

$\Longleftrightarrow$

$$
\beta>1-\frac{k_{0}^{*}}{N-k_{0}^{*}} .
$$

(B) Social planner's problem (78) requires $\frac{\partial W_{0}^{*}}{\partial k_{0}}=0$. Applying the envelope theorem, we have

$$
\frac{d W_{0}^{*}}{d \gamma}=\frac{\partial W_{0}^{*}}{\partial k_{0}} \frac{\partial k_{0}}{\partial \gamma}+\frac{\partial W_{0}^{*}}{\partial \gamma}=\int_{0}^{\infty} e^{-r t} \frac{\partial U\left(k_{t}\right)}{\partial \gamma} d t>0
$$

for any $\beta>0$. Similarly, we can prove $d W_{0}^{*} / d A>0, d W_{0}^{*} / d c<0$, and $d W_{0}^{*} / d r<0$.

\section{A.8. Proof of Proposition 8}

Proof. Given that condition (25) holds, we have $k_{0}^{*}<N$. Proposition 6 shows that the socially optimal innovation $k_{0}^{*}$ satisfies Eq. (24) that

$$
\int_{0}^{\infty} e^{-(r+\gamma N) t}\left(\frac{k_{t}}{k_{0}}\right)^{2} A k_{t}^{-\beta} d t=c .
$$


When $\alpha=0$, condition (25) is equivalent to condition (15). We have $k_{0}^{\mathbf{I}}=k_{0}^{\mathbf{I I}}<N$ and they satisfy Eq. (13) or Eq. (14) so that

$$
\begin{aligned}
& \int_{0}^{\infty} e^{-r t} \frac{1}{N-k_{0}}\left(\frac{N}{k_{t}}-1\right) A k_{t}^{1-\beta} d t=c . \\
& \int_{0}^{\infty} e^{-(r+\gamma N) t}\left(\frac{k_{0}}{k_{t}}\right)\left(\frac{k_{t}}{k_{0}}\right)^{2} A k_{t}^{-\beta} d t=c .
\end{aligned}
$$

Note that the left hand side of Eq. (79) is smaller than the left hand side of Eq. (24) given that $\frac{k_{0}}{k_{t}}<1$ for $t>0$. Therefore, the solution $k_{0}^{\mathbf{I}}=k_{0}^{\mathbf{I I}}$ to Eq. (79) would cause the left hand side of Eq. (24) greater than $c$. Because the left hand side of (24) decreases with $k_{0}$, the value of $k_{0}^{*}$ needs to be larger. Proposition $4(\mathrm{~A})$ shows that $\partial k_{0}^{\mathbf{I}} / \partial \alpha>0$ and $\partial k_{0}^{\mathbf{I I}} / \partial \alpha>0$, which implies that $\alpha^{\mathbf{I} *}>0$ and $\alpha^{\mathbf{I I} *}>0$.

When $\alpha=1$, if condition (15) holds, we have $k_{0}^{\mathbf{I}}=k_{0}^{\mathbf{I I}}<N$ and they satisfy Eq. (13) or Eq. (14) so that

$$
\begin{gathered}
\int_{0}^{\infty} e^{-(r+\gamma N) t}\left(\frac{k_{t}}{k_{0}}\right)^{2} A k_{t}^{-\beta} d t=c . \\
\int_{0}^{\infty} e^{-(r+\gamma N) t}\left(\frac{k_{0}}{N} e^{\gamma N t}-\frac{k_{0}}{N}+1\right)\left(\frac{k_{t}}{k_{0}}\right)^{2} A k_{t}^{-\beta} d t=c .
\end{gathered}
$$

Note that the left hand side of Eq. (80) is greater than the left hand side of Eq. (24) given that $\frac{k_{0}}{N} e^{\gamma N t}-\frac{k_{0}}{N}+1>1$ for $t>0$. Therefore, the solution $k_{0}^{\mathbf{I}}=k_{0}^{\mathbf{I I}}$ to Eq. (80) would cause the left hand side of (24) smaller than $c$. Because the left hand side of Eq. (24) decreases with $k_{0}$, the value of $k_{0}^{*}$ needs to be smaller. Proposition $4(\mathrm{~A})$ shows that $\partial k_{0}^{\mathbf{I}} / \partial \alpha>0$ and $\partial k_{0}^{\mathbf{I I}} / \partial \alpha>0$, therefore $\alpha^{\mathbf{I} *}<1$ and $\alpha^{\mathbf{I I} *}<1$. Note that in case condition (15) does not hold when $\alpha=1$, we have $k_{0}^{\mathbf{I}}=k_{0}^{\mathbf{I I}}=N$ and a smaller value of $\alpha<1$ is needed to achieve $k_{0}^{*}<N$.

Because Eqs. (13) and (14) are continuous functions, there exist $0<\alpha^{\mathbf{I} *}<1$ and $0<\alpha^{\mathbf{I I} *}<1$ to achieve $k_{0}^{*}$ in Regime 1 and 2, respectively. Proposition 4(B) shows that for any given $\alpha \in(0,1)$, we have $k_{0}^{\mathbf{I}}>k_{0}^{\mathbf{I I}}$. Accordingly, if the bargaining share $\alpha^{\mathbf{I} *}$ leads to the social optimum $k_{0}^{\mathbf{I}}=k_{0}^{*}$ in Regime 1, the same bargaining share would lead to a $k_{0}^{\mathbf{I I}}<k_{0}^{*}$ in Regime 2 . This implies that a higher bargaining share $\alpha^{\mathbf{I I} *}>\alpha^{\mathbf{I} *}$ is needed to achieve $k_{0}^{*}$ in Regime 2 given that $\partial k_{0}^{\mathbf{I I}} / \partial \alpha>0$ as shown by Proposition 4(A).

\section{A.9. Proof of Proposition 9}

Proof. In Regime 1, for a given value of $\alpha$, Eq. (13) yields the market equilibrium entry of innovators $k_{0}^{\mathbf{I}}$. Proposition 8 suggests that whenever $\alpha \neq \alpha^{\mathbf{I} *}$, the number of innovators $k_{0}^{\mathbf{I}}$ from Eq. (13) differs from the social optimum $k_{0}^{*}$, in which case offering an innovation subsidy (or tax) to adjust the innovation cost $c$ would help restore the 
social optimum. This implies that $k_{0}^{*}$ can be achieved by a subsidy (or a tax if the subsidy is negative) $s^{\mathbf{I} *}$ as follows:

$$
\frac{1}{N-k_{0}^{*}} \int_{0}^{\infty} e^{-r t}\left(\alpha \frac{N}{k_{0}^{*}}+(1-\alpha) \frac{N}{k_{t}^{*}}-1\right) A k_{t}^{* 1-\beta} d t=c-s^{\mathbf{I} *} .
$$

The same logic applies to Regime 2 that

$$
\frac{1}{N-k_{0}^{*}} \int_{0}^{\infty} e^{-r t}\left(\left(\frac{N}{k_{0}^{*}}\right)^{\alpha}\left(\frac{N}{k_{t}^{*}}\right)^{1-\alpha}-1\right) A k_{t}^{* 1-\beta} d t=c-s^{\mathbf{I I} *} .
$$

Recall that when $\alpha \in\{0,1\}$, Regimes 1 and 2 coincide. When $\alpha=0$, both regimes would need more entry of innovators, so a positive subsidy is needed to achieve that, and when $\alpha=1$, a negative subsidy (tax) is needed. Moreover, for $\alpha \in(0,1)$, according to Proposition 4(B), if a given pair of $\alpha$ and $\left(c-s^{\mathbf{I} *}\right)$ lead to the social optimum $k_{0}^{*}$ in Regime 1 , the same parameter values would result in a $k_{0}^{\mathbf{I I}}<k_{0}^{*}$ in Regime 2. Therefore, a higher subsidy (or a smaller tax) $s^{\mathbf{I I} *}$ is needed for adjusting $c$ to achieve $k_{0}^{*}$ in Regime 2 given that $k_{0}^{\mathbf{I I}}$ decreases with $c$ as shown by Proposition $4(\mathrm{~A})$.

\section{A.10. Proof of Proposition 10}

Proof. This is a special case of Proposition 11 by taking $\alpha=0$, and Regimes 1 and 2 coincide in this case.

\section{A.11. Proof of Proposition 11}

Proof. With $\beta=1$ under Regime 1, Eq. (13) can be simplified as

$$
k_{0}^{\mathbf{I}}=\frac{A(\alpha \gamma N+r)}{c r(r+\gamma N)} .
$$

Given Eq. (81) and $\beta=1$, social surplus is

$W_{0}=-\frac{A(1-\alpha)}{(r+\gamma N)}+A \int_{0}^{\infty} e^{-r t}\left[\gamma N t-\ln \left(e^{\gamma N t}+\frac{N c r(r+\gamma N)}{A(\alpha \gamma N+r)}-1\right)\right] d t+$ constant.

This suggests that

$$
\frac{d W_{0}}{d \gamma}=\frac{A(1-\alpha) N}{(r+\gamma N)^{2}}+A \int_{0}^{\infty} e^{-r t} N t d t-A \int_{0}^{\infty} e^{-r t}\left[\frac{N t e^{\gamma N t}+\frac{c r N^{2}}{A(\alpha \gamma N+r)}-\frac{\alpha c r(r+\gamma N) N^{2}}{A(\alpha \gamma N+r)^{2}}}{e^{\gamma N t}+\frac{N c r(r+\gamma N)}{A(\alpha \gamma N+r)}-1}\right] d t
$$


We then verify that

$$
\begin{aligned}
& \left.d\left[\frac{\left.N t e^{\gamma N t}+\frac{c r N^{2}}{A(\alpha \gamma N+r)}-\frac{\alpha c r(r+\gamma N) N^{2}}{A(\alpha \gamma N+r)^{2}}\right] / d c}{e^{\gamma N t}+\frac{N c r(r+\gamma N)}{A(\alpha \gamma N+r)}-1}\right] / \frac{N c r(r+\gamma N)}{A(\alpha \gamma N+r)}-1\right) \\
\propto & \left(\frac{(1-\alpha) r}{(\alpha \gamma N+r)}\right)\left(e^{\gamma N t}+\frac{\alpha+\gamma}{\alpha r}(r+\gamma N) N\right. \\
& -\left(t e^{\gamma N t}+\frac{c r N}{A(\alpha \gamma N+r)}-\frac{\alpha(\alpha \gamma N+r)^{2}}{A(\alpha+\gamma N)}\right) \\
< & 0
\end{aligned}
$$

for any $t>0$. Equation (81) implies that $c>\frac{A(\alpha \gamma N+r)}{N r(r+\gamma N)}$ for $k_{0}^{\mathbf{I}}$ to be interior solution (i.e., $\left.k_{0}^{\mathbf{I}}<N\right)$. Accordingly, given that $c>\frac{A(\alpha \gamma N+r)}{N r(r+\gamma N)}$, we have

$A \int_{0}^{\infty} e^{-r t}\left[\frac{N t e^{\gamma N t}+\frac{c r N^{2}}{A(\alpha \gamma N+r)}-\frac{\alpha c r(r+\gamma N) N^{2}}{A(\alpha \gamma N+r)^{2}}}{e^{\gamma N t}+\frac{N c r(r+\gamma N)}{A(\alpha \gamma N+r)}-1}\right] d t<A \int_{0}^{\infty} e^{-r t}\left[\frac{N t e^{\gamma N t}+\frac{N}{r+\gamma N}-\frac{\alpha N}{(\alpha \gamma N+r)}}{e^{\gamma N t}}\right] d t$

Therefore,

$$
\begin{aligned}
\frac{d W_{0}}{d \gamma} & >\frac{A(1-\alpha) N}{(r+\gamma N)^{2}}+A \int_{0}^{\infty} e^{-r t} N t d t-A \int_{0}^{\infty} e^{-r t}\left[\frac{N t e^{\gamma N t}+\frac{N}{r+\gamma N}-\frac{\alpha N}{(\alpha \gamma N+r)}}{e^{\gamma N t}}\right] d t \\
& =\frac{A(1-\alpha) N}{(r+\gamma N)^{2}}-\frac{A}{(r+\gamma N)}\left(\frac{N}{r+\gamma N}-\frac{\alpha N}{(\alpha \gamma N+r)}\right) \\
& =\frac{A \alpha N}{(r+\gamma N)}\left(\frac{1}{r+\alpha \gamma N}-\frac{1}{r+\gamma N}\right) \geq 0 \text { for any } \alpha \in[0,1] .
\end{aligned}
$$

\section{A.12. Proof of Proposition 12}

Proof. Equations (3) and (4) imply that for given $k_{t}$,

$$
\frac{d k_{t} / d t}{k_{t}}=\gamma\left(N-k_{t}\right)=\gamma N\left(1-\frac{e^{\lambda t}}{e^{\lambda t}+\frac{N}{k_{0}}-1}\right) .
$$

Given that the demand curve is downward slopping, $k_{0}$ has to be finite as $N \rightarrow \infty$; otherwise $p_{0} \rightarrow 0$, and no innovator would enter at date 0 . Therefore, Eq. (82) implies that

$$
\left.\frac{d k_{t} / d t}{k_{t}}\right|_{N \rightarrow \infty} \rightarrow \lambda
$$

i.e., the incumbents' meeting rate converges to a constant. 


\section{A.13. Proof of Proposition 13}

Proof. Assume that condition (44) holds (i.e., $\lambda<r$ ) so that social welfare derived from the innovation is bounded. We start with the conjecture that no agent would enter as an innovator after date 0, so firm numbers evolve as described by Eq. (43). Given that an imitator cannot resell the idea, his only revenue comes from selling the good, and his value $\omega_{t}$ satisfies the ordinary differential equation (ODE):

$$
r \omega_{t}=p_{t}+\frac{d \omega_{t}}{d t}=A\left(k_{0} e^{\lambda t}\right)^{-\beta}+\frac{d \omega_{t}}{d t} .
$$

The ODE has the unique bounded solution

$$
\omega_{t}=\frac{A k_{0}^{-\beta}}{r+\beta \lambda} e^{-\beta \lambda t},
$$

which decreases at the rate $\beta \lambda$.

An innovator receives revenues from selling both the good and the idea. The number of ideas sold at $t$ is $\lambda k_{t}$ and the total date- $t$ revenue from these sales, $\lambda k_{t} \alpha \omega_{t}$ is divided among the $k_{0}$ innovators. Thus $v_{t}$, the value of being an innovator at date $t$, follows the ODE:

$$
r v_{t}=p_{t}+\frac{\lambda k_{t}}{k_{0}} \alpha \omega_{t}+\frac{d v_{t}}{d t}=A\left(k_{0} e^{\lambda t}\right)^{-\beta}+\frac{\alpha \lambda A k_{0}^{-\beta} e^{(1-\beta) \lambda t}}{r+\beta \lambda}+\frac{d v_{t}}{d t} .
$$

Unless $\alpha=0$, innovators receive a fraction of revenues from idea sales, and we shall need to restrict the elasticity of demand to be below unity which means $\beta \geq 1$. Imposing the boundary condition $v_{t \rightarrow \infty}<\infty$ yields the unique solution to Eq. (86):

$$
v_{t}=\frac{A k_{0}^{-\beta} e^{-\beta \lambda t}}{r+\beta \lambda}+\frac{\alpha \lambda A k_{0}^{-\beta}}{(r+\beta \lambda)(r+(\beta-1) \lambda)} e^{-(\beta-1) \lambda t} .
$$

Recall that $u_{t}$ denotes the option value of becoming a future imitator. At $t=0$, the free entry condition requires $v_{0}-u_{0}=c$. The pool of outsiders being infinite, an outsider's chance of meeting an incumbent is zero so that $u_{t}=0$ for all $t$, implying that $v_{0}=c$. Since $v_{t}$ decreases over time, we verify the conjecture that no one would pay $c$ to become an innovator at any date $t>0$. Note that if an agent deviates from the equilibrium and enters at date $t>0$, he would have a lower valuation than an innovator who entered at date 0 (i.e., $v_{t}^{t}<v_{t}^{0}$ ) because the latter would have a larger family of imitators to disseminate his idea and collect idea-sale revenues. Therefore, the finding that $v_{t}-u_{t}$ declines in $t$ implies that $v_{t}^{t}-u_{t}<c$ at any date $t>0$.

Combining $v_{0}=c$ with Eq. (87) yields

$$
v_{0}=\frac{A k_{0}^{-\beta}}{r+\beta \lambda}+\frac{\alpha \lambda A k_{0}^{-\beta}}{(r+\beta \lambda)(r+(\beta-1) \lambda)}=c .
$$


Equation (88) then determines the entry of innovators at date 0 to be

$$
k_{0}^{\mathbf{I}}=\left(\frac{A(r+(\beta-1) \lambda+\alpha \lambda)}{c(r+\beta \lambda)(r+(\beta-1) \lambda)}\right)^{\frac{1}{\beta}},
$$

which is valid for $\beta \geq 1$ or for all $\beta>0$ if $\alpha=0$.

\section{A.14. Proof of Proposition 14}

Proof. Assume that condition (44) holds (i.e., $\lambda<r$ ) so that social welfare derived from the innovation is bounded. We conjecture that no agent would enter as an innovator after date 0 . Given that imitators can resell the innovation, all the incumbents (be they innovators or imitators) share the same value $v_{t}$. The revenue from an idea sale is $\alpha v_{t}$ and the total date- $t$ revenue from these sales, $\lambda k_{t} \alpha v_{t}$, is shared equally among all the incumbents. Then $v_{t}$ follows the ODE:

$$
r v_{t}=p_{t}+\lambda \alpha v_{t}+\frac{d v_{t}}{d t}=A\left(k_{0} e^{\lambda t}\right)^{-\beta}+\lambda \alpha v_{t}+\frac{d v_{t}}{d t}
$$

The general solution of Eq. (90) is

$$
v_{t}=\frac{A k_{0}^{-\beta} e^{-\beta \lambda t}}{r+\beta \lambda-\lambda \alpha}+A k_{0}^{-\beta} C e^{(r-\lambda \alpha) t},
$$

where $C$ is a constant of integration. Given that $\lambda<r$, the boundary condition $v_{t \rightarrow \infty}<\infty$ requires $C=0$ and yields

$$
v_{t}=\frac{A k_{0}^{-\beta} e^{-\beta \lambda t}}{r+(\beta-\alpha) \lambda} .
$$

Equation (91) shows $v_{t}$ decreases with $t$. Also, because the pool of outsiders is infinite, an outsider's chance of meeting an incumbent is zero so that $u_{t}=0$ for all $t$. Hence, we verify the conjecture that innovators only enter at $t=0$. At $t=0$, we again have $v_{0}=c$, thus

$$
v_{0}=\frac{A k_{0}^{-\beta}}{r+(\beta-\alpha) \lambda}=c .
$$

Equation (92) then determines the entry of innovators at date 0 to be

$$
k_{0}^{\mathbf{I I}}=\left(\frac{A}{c(r+(\beta-\alpha) \lambda)}\right)^{\frac{1}{\beta}} .
$$




\section{A.15. Proof of Proposition 15}

Proof. (A) It is straightforward to verify from Eqs. (89) and (93) that

$$
\begin{aligned}
& \partial k_{0}^{\mathbf{I}} / \partial \alpha>0, \quad \partial k_{0}^{\mathbf{I}} / \partial A>0, \quad \partial k_{0}^{\mathbf{I}} / \partial c<0, \quad \partial k_{0}^{\mathbf{I}} / \partial r<0, \\
& \partial k_{0}^{\mathbf{I I}} / \partial \alpha>0, \quad \partial k_{0}^{\mathbf{I I}} / \partial A>0, \quad \partial k_{0}^{\mathbf{I I}} / \partial c<0, \quad \partial k_{0}^{\mathbf{I I}} / \partial r<0 .
\end{aligned}
$$

Moreover, Eq. (89) shows $d k_{0}^{\mathbf{I}} / d \lambda<0$ for $\beta \geq 1>\alpha$ or for any $\beta>\alpha=0$, and $d k_{0}^{\mathbf{I}} / d \lambda=0$ for $\beta=\alpha=1$. Equation (93) shows $d k_{0}^{\mathbf{I I}} / d \lambda \lesseqgtr 0$ if $\beta \gtreqless \alpha$.

The effect of $\lambda$.-The effect of diffusion speed on innovation depends on $\alpha$ and $\beta$. Specifically, in Eq. (93), $k_{0}^{\text {II }}$ falls with $\lambda$ if $\beta>\alpha$ because the effect of entry of competitors on reducing $p_{t}$ (summarized by $\beta$ ) exceeds the benefit (summarized by $\alpha$ ) that incumbents derive from selling the idea. The effect of $\lambda$ on $k_{0}^{\text {II }}$ turns positive if $\beta<\alpha$, and it vanishes if $\beta=\alpha$. In Regime 1 where imitators cannot resell ideas (cf. Eq. (89)), $k_{0}^{\mathbf{I}}$ falls with $\lambda$ for $\beta \geq 1>\alpha$ or for any $\beta>\alpha=0$, and the effect of $\lambda$ on $k_{0}^{\mathbf{I}}$ vanishes when $\beta=\alpha=1$.

(B) Equations (89) and (93) imply that

$$
\left(\frac{k_{0}^{\mathbf{I}}}{k_{0}^{\mathbf{I I}}}\right)^{\beta}=1+\frac{\alpha(1-\alpha) \lambda^{2}}{(r+\beta \lambda)(r+(\beta-1) \lambda)}=\left\{\begin{array}{cr}
1 & \text { for } \alpha \in\{0,1\} \\
>1 & \text { for } \alpha \in(0,1)
\end{array} .\right.
$$

\section{A.16. Proof of Proposition 16.}

Proof. Recall that the planner would like to maximize social welfare

$$
W_{0}=\int_{0}^{\infty} e^{-r t} U\left(k_{t}\right) d t-c k_{0},
$$

where

$$
U\left(k_{t}\right)= \begin{cases}\frac{A}{1-\beta} k_{t}^{1-\beta} & \text { if } \beta \in(0,1) \\ A\left(\ln k_{t}+1-\ln \varepsilon\right) & \text { if } \beta=1, \\ \frac{A}{1-\beta} k_{t}^{1-\beta}+\frac{\beta A}{\beta-1} \varepsilon^{1-\beta} & \text { if } \beta>1 .\end{cases}
$$

and

$$
k_{t}=k_{0} e^{\lambda t} .
$$

We assume that condition (44) holds (i.e., $\lambda<r$ ) so that social welfare $W_{0}$ is bounded.

(A) Consider the case $\beta<1$ first. For any date $\tau \geq 0$, if no further innovators enter, the number of firms at dates $t \geq \tau$ is

$$
k_{t}=k_{\tau} e^{\lambda(t-\tau)} \quad \text { for } t \geq \tau .
$$

As of date $\tau$, the planner's return to innovation is

$$
S R_{\tau}=\int_{\tau}^{\infty} e^{-r(t-\tau)} \frac{A}{1-\beta} k_{t}^{1-\beta} d t .
$$


The current cost of innovation is $c$ per unit, and its marginal social return (even if no further innovations are made) is

$$
\frac{\partial S R_{\tau}}{\partial k_{\tau}}=\int_{\tau}^{\infty} e^{-r(t-\tau)} A k_{t}^{-\beta} \frac{\partial k_{t}}{\partial k_{\tau}} d t=\int_{\tau}^{\infty} e^{-(r-\lambda)(t-\tau)} A k_{t}^{-\beta} d t .
$$

Define $s=t-\tau$, we can rewrite Eq. (95) as

$$
\frac{\partial S R_{\tau}}{\partial k_{\tau}}=\int_{0}^{\infty} e^{-(r-\lambda) s} A k_{\tau+s}^{-\beta} d s=\int_{0}^{\infty} e^{-(r-\lambda) s} A\left(k_{\tau} e^{\lambda s}\right)^{-\beta} d s,
$$

which is strictly decreasing in $k_{\tau}$. Therefore, if at date zero $k_{0}$ is chosen so that $\frac{\partial S R_{0}}{\partial k_{0}}=c$, thereafter $\frac{\partial S R_{\tau}}{\partial k_{\tau}}<c$. Similarly, we can prove the result holds for $\beta \geq 1$. Hence, it is socially optimal to innovate only at date 0 .

(B) The social planner chooses $k_{0}$ to maximize social welfare:

$$
W_{0}=\int_{0}^{\infty} e^{-r t} U\left(k_{t}\right) d t-c k_{0} .
$$

We verify that the social welfare function is strictly concave in $k_{0}$, so the first-order condition yields the socially optimal number of innovators $k_{0}^{*}$ :

$$
k_{0}^{*}=\left(\frac{A}{c(r+(\beta-1) \lambda)}\right)^{\frac{1}{\beta}} .
$$

(C) Comparing (96) to the expressions in Eqs. (89) and (93), $k_{0}^{*}=k_{0}^{\mathbf{I}}=k_{0}^{\mathbf{I I}}$ iff $\alpha=1$.

\section{A.17. Proof of Proposition 17.}

Proof. Recall that the solutions for $k_{0}^{\mathbf{I}}, k_{0}^{\mathbf{I I}}$ and $k_{0}^{*}$ are given by Propositions 13,14 and 16, respectively. Given that the optimal compensation for idea sellers is $\alpha^{*}=1$, the planner could improve social welfare by offering an innovation subsidy $s$ whenever $\alpha<1$. Accordingly, the net entry cost of innovators becomes $c-s$. Under Regime 1, Eqs. (89) and (96) pin down the optimal subsidy $s^{\mathbf{I} *}$ so that

$$
c[r+(\beta-1) \lambda+\alpha \lambda]=\left(c-s^{\mathbf{I} *}\right)(r+\beta \lambda),
$$

which yields

$$
s^{\mathbf{I}}=\frac{c(1-\alpha) \lambda}{r+\beta \lambda} .
$$

Under Regime 2, Eqs. (93) and (96) pin down the optimal subsidy $s^{\mathbf{I I} *}$ so that

$$
c[r+(\beta-1) \lambda]=\left(c-s^{\mathbf{I I} *}\right)[r+(\beta-\alpha) \lambda],
$$


which yields

$$
s^{\mathbf{I I} *}=\frac{c(1-\alpha) \lambda}{r+(\beta-\alpha) \lambda} .
$$

The result (97) and (98) suggest that in the limiting model, $0<s^{\mathbf{I} *}<s^{\mathbf{I I} *}$ for any $0<\alpha<1 ; s^{\mathbf{I} *}=s^{\mathbf{I I} *}=0$ for $\alpha=1$; and $s^{\mathbf{I} *}=s^{\mathbf{I I} *}=\frac{c \lambda}{r+\beta \lambda}$ for $\alpha=0$.

\section{A.18. Proof of Proposition 18}

Proof. Recall that the solutions for $k_{0}^{\mathbf{I}}$ and $k_{0}^{\mathbf{I I}}$ are given by Propositions 13 and 14, respectively. According to Eqs. (89) and (93),

$$
k_{0}^{\mathbf{I}}=\left(\frac{A(r+(\beta-1) \lambda+\alpha \lambda)}{c(r+\beta \lambda)(r+(\beta-1) \lambda)}\right)^{\frac{1}{\beta}} ; \quad k_{0}^{\mathbf{I I}}=\left(\frac{A}{c(r+(\beta-\alpha) \lambda)}\right)^{\frac{1}{\beta}} .
$$

In each regime, the number of firms grows at a constant rate $\lambda$ (i.e., $k_{t}^{\mathbf{I}}=k_{0}^{\mathbf{I}} e^{\lambda t}$ and $\left.k_{t}^{\mathbf{I I}}=k_{0}^{\mathbf{I I}} e^{\lambda t}\right)$. The planner would like to maximize social welfare

$$
W_{0}=\int_{0}^{\infty} e^{-r t} U\left(k_{t}\right) d t-c k_{0}
$$

where

$$
U\left(k_{t}\right)= \begin{cases}\frac{A}{1-\beta} k_{t}^{1-\beta} & \text { if } \beta \in(0,1), \\ A\left(\ln k_{t}+1-\ln \varepsilon\right) & \text { if } \beta=1, \\ \frac{A}{1-\beta} k_{t}^{1-\beta}+\frac{\beta A}{\beta-1} \varepsilon^{1-\beta} & \text { if } \beta>1 .\end{cases}
$$

We assume that condition (44) holds (i.e., $\lambda<r$ ) so that social welfare $W_{0}$ is bounded.

Denote $W_{0}^{\mathbf{I}}$ and $W_{0}^{\mathbf{I I}}$ as the social welfare under Regimes 1 and 2, respectively. With free knowledge spillovers $(\alpha=0)$, we have $W_{0}^{\mathbf{I}}=W_{0}^{\mathbf{I I}}=W_{0}$, where

$$
W_{0}= \begin{cases}A^{\frac{1}{\beta}} c^{1-\frac{1}{\beta}}\left(\frac{(r+\beta \lambda)^{1-\frac{1}{\beta}}}{(1-\beta)(r-\lambda(1-\beta))}-(r+\beta \lambda)^{-\frac{1}{\beta}}\right) & \text { if } \beta \in(0,1), \\ \frac{A}{r} \ln \frac{A}{c(r+\lambda)}+\frac{A \lambda}{r^{2}}-\frac{A}{(r+\lambda)}+\frac{A(1-\ln \varepsilon)}{r} & \text { if } \beta=1, \\ A^{\frac{1}{\beta}} c^{1-\frac{1}{\beta}}\left(\frac{(r+\beta \lambda)^{1-\frac{1}{\beta}}}{(1-\beta)(r-\lambda(1-\beta))}-(r+\beta \lambda)^{-\frac{1}{\beta}}\right)+\frac{\beta A}{r(\beta-1)} \varepsilon^{1-\beta} & \text { if } \beta>1 .\end{cases}
$$

It is straightforward to show that for any $\beta>0, \frac{\partial W_{0}}{\partial \lambda}>0$.

This finding can be extended to any $\alpha \in(0,1]$, for which we have

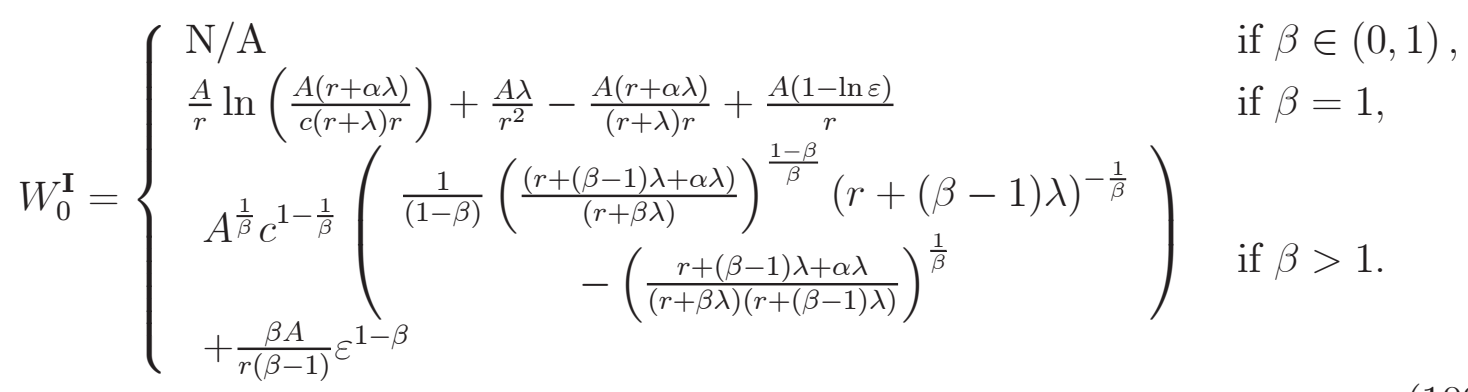




$$
W_{0}^{\mathbf{I I}}= \begin{cases}A^{\frac{1}{\beta}} c^{1-\frac{1}{\beta}}\left(\frac{(r+(\beta-\alpha) \lambda)^{1-\frac{1}{\beta}}}{(1-\beta)(r-\lambda(1-\beta))}-(r+(\beta-\alpha) \lambda)^{-\frac{1}{\beta}}\right) & \text { if } \beta \in(0,1), \\ \frac{A}{r} \ln \frac{A}{c(r+(1-\alpha) \lambda)}+\frac{A \lambda}{r^{2}}-\frac{A}{r+(1-\alpha) \lambda}+\frac{A}{r}(1-\ln \varepsilon) & \text { if } \beta=1, \\ A^{\frac{1}{\beta}} c^{1-\frac{1}{\beta}}\left(\frac{(r+(\beta-\alpha) \lambda)^{1-\frac{1}{\beta}}}{(1-\beta)(r-\lambda(1-\beta))}-(r+(\beta-\alpha) \lambda)^{-\frac{1}{\beta}}\right) & \text { if } \beta>1 . \\ +\frac{\beta A \varepsilon^{1-\beta}}{r(\beta-1)} & \end{cases}
$$

We then confirm from Eqs. (100) and (101) that $\frac{\partial W_{0}^{\mathbf{I}}}{\partial \lambda}>0$ for $\beta \geq 1$ and $\frac{\partial W_{0}^{\text {II }}}{\partial \lambda}>0$ for $\beta>0$.

\section{B. Regressions.}

\section{B.1. Auto diffusion estimation: Robustness checks}

For robustness checks, we estimate the matching function directly by rewriting Eq. (3) into a discrete-time version:

$$
\frac{k_{t}-k_{t-1}}{N-k_{t-1}}=\gamma k_{t-1}
$$

Note that the left-hand side of Eq. (102) is the hazard rate of adopting the new product. We set $N=210$ and run the regression model (102) using auto firm number data from 1895-1908. The result shows that

$$
\frac{k_{t}-k_{t-1}}{N-k_{t-1}}=\underset{(0.0004)^{* * *}}{0.0028} k_{t-1},
$$

and the standard error is reported in the parentheses. The estimate of $\gamma$ is statistically significant at $1 \%$ level and adjusted $R^{2}=0.77$. The estimate $\gamma=0.0028$ implies that $\gamma N=0.59$, which is similar to the estimate from Eq. (34).

We also redo the exercise by estimating an extended version of Eq. (102) that

$$
\frac{k_{t}-k_{t-1}}{N-k_{t-1}}=\eta+\gamma k_{t-1}
$$

proposed by Bass (1969). The Bass model allows the hazard rate of adoption to be influenced by both the coefficient of innovation $\eta$ and the coefficient of imitation $\gamma$. In our context, $\eta$ captures the hazard rate of entry by innovators independent of incumbents while $\gamma k_{t-1}$ captures the hazard rate of entry by imitators. The regression result shows that

$$
\frac{k_{t}-k_{t-1}}{N-k_{t-1}}=\underset{(0.069)}{-0.0292}+\underset{(.0007)^{* * *}}{.0031} k_{t-1}
$$

and the standard errors are reported in the parentheses. The estimate $\gamma=0.0031$ (which implies $\gamma N=0.64$ ) is statistically significant at $1 \%$ level, but the estimate 
of $\eta$ is not statistically significant, which is consistent with our theoretical prediction that innovators only enter at the beginning of the industry.

\section{B.2. Auto demand estimation: Robustness checks}

For robustness checks, we estimate the auto industry demand function by controlling for changes of population and per capita income over time. In doing so, we use annual data of auto prices $p_{t}$ and output $Q_{t}$ from 1900-1929 to estimate a per capita demand function:

$$
\ln \left(\frac{Q_{t}}{\text { pop }_{t}}\right)=a_{t}-\phi \ln \left(p_{t}\right)
$$

The dependent variable is auto demand per capita (where pop $_{t}$ is U.S. population at year $t$ ), and we control for log U.S. GDP per capita (as a proxy for income) in the demand intercept $a_{t}$. Both auto price and GDP per capita are in real terms.

As before, to address potential endogeneity of the price variable, we use the output per firm (lagged by a year) as an instrumental variable to estimate the demand elasticity parameter $\phi$ in a two-stage least-squares regression.

The first-stage regression result (adj. $\left.R^{2}=0.89\right)$ is given by

$$
\ln \left(p_{t}\right)=\underset{(1.09)^{* * *}}{8.56}+\underset{(0.64)^{* *}}{1.66} \times \ln \left(\frac{G D P_{t}}{\text { pop }_{t}}\right)-\underset{(0.03)^{* * *}}{0.29} \times \ln (\text { output per firm })_{t-1},
$$

and the second-stage regression result $\left(R^{2}=0.83\right)$ is

$$
\ln \left(\frac{Q_{t}}{\text { pop }_{t}}\right)=\underset{(7.16)^{* * *}}{32.37}+\underset{(2.10)}{0.28} \times \ln \left(\frac{G D P_{t}}{\text { popt }_{t}}\right)-\underset{(0.38)^{* * *}}{3.33} \times \ln \left(p_{t}\right) .
$$

Standard errors are reported in the parentheses, with three stars and two stars representing statistical significance at $1 \%$ and $5 \%$ level, respectively. The estimate $\phi=3.33$ is highly statistically significant and the implied inverse demand elasticity $\beta=\frac{1}{\phi}=0.3$ is similar to the estimate from Eq. (35).

\section{B.3. PC diffusion estimation: Robustness checks}

For robustness checks, we estimate the matching function (102) for the PC industry:

$$
\frac{k_{t}-k_{t-1}}{N-k_{t-1}}=\gamma k_{t-1}
$$

We set $N=435$ and run the regression using PC firm number data from 1975-1991. The result shows that

$$
\frac{k_{t}-k_{t-1}}{N-k_{t-1}}=\underset{(0.00025)^{* * *}}{0.00092} k_{t-1},
$$

and the standard error is reported in the parentheses. The estimate of $\gamma$ is statistically significant at $1 \%$ level and adjusted $R^{2}=0.44$. The estimate $\gamma=0.00092$ implies that $\gamma N=0.40$. 
We also redo the exercise by estimating a more general version (cf. Eq. (103)) that

$$
\frac{k_{t}-k_{t-1}}{N-k_{t-1}}=\eta+\gamma k_{t-1}
$$

proposed by Bass (1969). In our context, $\eta$ captures the hazard rate of entry by innovators independent of incumbents, while $\gamma k_{t-1}$ captures the hazard rate of entry by imitators. The regression result shows that

$$
\frac{k_{t}-k_{t-1}}{N-k_{t-1}}=\underset{(0.08398)}{0.04721}+\underset{(0.00036)^{*}}{0.00078 k_{t-1}}
$$

and the standard errors are reported in the parentheses. The estimate $\gamma=0.00078$ (which implies $\gamma N=0.34$ ) is statistically significant at $5.1 \%$ level, but the estimate of $\eta$ is not statistically significant, which is consistent with our theoretical prediction that innovators only enter at the beginning of the industry.

\section{B.4. PC demand estimation: Robustness checks}

For robustness checks, we estimate the PC industry demand function by controlling for changes of population and per capita income over time. In doing so, we use annual data of PC prices $p_{t}$ and output $Q_{t}$ from 1975-1992 to estimate a per capita demand function:

$$
\ln \left(\frac{Q_{t}}{\text { pop }_{t}}\right)=a_{t}-\phi \ln \left(p_{t}\right)
$$

The dependent variable is PC demand per capita (where $p o p_{t}$ is U.S. population at year $t$ ), and we control for log U.S. GDP per capita (as a proxy for income) in the demand intercept $a_{t}$. Both auto price and GDP per capita are in real terms.

As before, to address potential endogeneity of the price variable, we use the output per firm (lagged by a year) as an instrumental variable to estimate the demand elasticity parameter $\phi$ in a two-stage least-squares regression.

The first-stage regression result (adj. $\left.R^{2}=0.95\right)$ is given by

$$
\ln \left(p_{t}\right)=\underset{(0.23)^{* * *}}{12.44}-\underset{(0.06)^{* * *}}{0.95} \times \ln \left(\frac{G D P_{t}}{\text { opp }_{t}}\right)-\underset{(0.01)^{* * *}}{0.07} \times \ln (\text { output per firm })_{t-1},
$$

and the second-stage regression result $\left(R^{2}=0.95\right)$ is

$$
\ln \left(\frac{Q_{t}}{\text { pop }_{t}}\right)=\underset{(29.00)^{* * *}}{143.18}-\underset{(2.63)}{2.90} \times \ln \left(\frac{G D P_{t}}{\text { pop }_{t}}\right)-\underset{(2.40)^{* * *}}{15.57} \times \ln \left(p_{t}\right) .
$$

Standard errors are reported in the parentheses, with three stars indicating statistical significance at $1 \%$ level. The estimate $\phi=15.57$ is highly statistically significant and the implied inverse demand elasticity $\beta=\frac{1}{\phi}=0.06$ is similar to the estimate from Eq. (37). 